Universidade de São Paulo

Instituto de Física

Departamento de Física Matemática

\title{
Análise Hamiltoniana e Quantização da Teoria de Gravitação Dilatônica em Duas Dimensōes
}

\author{
Tese de Doutoramento \\ submetida ao Instituto de Física \\ da Universidade de Säo Paulo
}

Sebastião Cassemiro de Figueiredo Filho

Banca Examinadora: Prof. Dr. Henrique Fleming (USP)

Prof. Dr. Josif Frenkel (USP)

Prof. Dr. A. Zimerman (IFT)

Prof. Dr. J. A. Helayel Neto (CBPF)

Prof. Dr. Victgr fo Rivelles (USP)

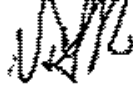

Orientador: Prof. Victor O. Rivelles

Săo Paulo

1999

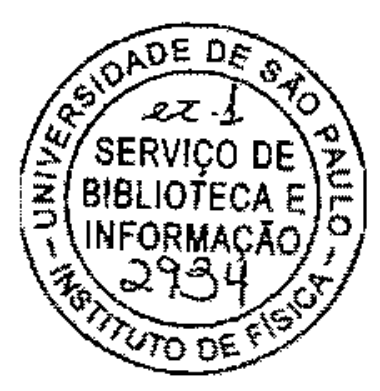


FICHA CATALOGRÁFICA

Preparada pelo Serviço de Biblioteca e Informaçåo do Instituto de Física da Universidade de São Paulo

Figueiredo Filho, Sebastiẩo Cassemiro de

Análise Hamiltoniana e quantização da teoria de gravitação dilatônica em duas dimensões. São Paulo, 1999.

Tese (Doutorado) Universidade de São Paulo. Instituto de Fisica - Departamento de Fisica Matemática.

Orientador: Prof. Dr. Victor O. Rivelles

Área de Concentraçäo:Fisica de Partículas Elementares

Unitermos:1. Gravitação em 2D; 2.Modelos dilatỏnicos em 2D; 3. Modelo de CGHS

USPIF/SBI-013/99 


\section{Agradecimentos}

Ao Prof V.O.Rivelles pelo trabalho proposto.

Ao colega L.Bufton pelas sugestöes no trabalho de ediçăo de texto.

A CAPES e as CNPa pelo suporte financeira. 


\begin{abstract}
We analyse a theory of gravitation in two dimensions using the hamiltonian formalism. For this purpose we use the CGHS model in the conformal gauge. The theory of constraints shows that we have only first-class constraints. After that, we apply the BRST-BFV technique to quantize the theory. We show that the ghosts in the partition function are decoupled from the fields. Then we integrate the ghosts in the partition function. By a suitable canonical transformation we write the constraints in a quadratic form which facilitates the quantization of the theory. To complete the quantization we use the Dirac procedure to obtain the constraints algebra expressed in the energy-momentum tensor form. There is an anomaly in the algebra which can be suitably eliminated by the addition of an improvement term in the action. Finally we use the Dirac procedure to obtain solutions for the wave function. This proves that the CGHS theory in two dimensions is quantizable.
\end{abstract}




\section{Resumo}

Neste trabalho, analisamos o modelo de gravitação dilatônica proposto por Callan, Giddings, Harvey e Strominger (modelo de CGHS), Aplicamos teoria de vinculos 20 modelo e mostramos que sô existen vínculos de primeira classe. Em seguida aplíamos a técnica de BRST-BFV para quantizar a teoria. Mostramos que os fantasmas na funçäo de partiçăo estăo desacoplados dos campos e poden ser integrados. Utilizamos uma transformação canônica para escrever os vínculos na forma quadrática, que faciltta a quantizaçăo da teoria. Para completar a quantização usamos o procedimento de Dirac para obter a algebra dos vínculos expressa na forma do tensor momento-energia. Existe uma anomatia nq ålgebra que é apropriadamente eliminada quando adicionamos um temo de improvement funçăo de onda. Isto prova que a teoria de CCHS em duas dimensŏes é perfeitamente quantizável. 


\section{Conteúdo}

1 INTRODUÇO

2 MODELO DE CGHS

2.1 Descriçăo do Modelo . . . . . . . . . . . . . . . . 9

2.2 Sistemas vinculados . . . . . . . . . . . . . . . . 11

2.3 Teoria de Sistemas Vinculados Aplicada ato Modelo de CGHS . . . . . . . 18

3 QUANTIZACÃO DO MODELO DE CGHS 20

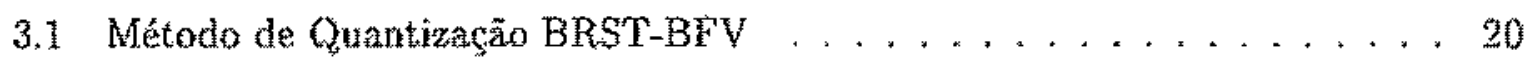

3.2 Quantizaçäo BRST-BFV do Modelo de CGHS . . . . . . . . . 24

3.3 Funçäo de Partição para o Modelo de CGHS no Gauge Conforme . . . 27

3.4 Forma Quadrática do Modelo de CGHS . . . . . . . . . . . 32

3.5 Quantizaçăo BRST-BFV do Modelo de CCHS na Forma Quadrática. . . . 34

3.6 Análise de Anomalias para o Modelo de CGHS . . . . . . . . . . 40

3.6 .1 Cálculo da Anomalia . . . . . . . . . . . . . . . 40

3.6.2 Termo de Improvement para o Cancelamento da Anomalia . . . . 48

3.6 .3 Quantizaçăo de Dirac para o Modelo de CGHS . . . . . . . 55

4 Conclusäo 63

A Parêntesez de Poisson $\quad 65$

B Tensor Momento Bnergia para os Fantasmas 67

C Álgebra do Tensor Momento-Energia para o Campo Escalar 71 
D Álgebra do Tensor Momento-Energia para os Fantasmas

E Algebra do Tensor Momento-Energia para o Campo Escalar + Termo de Improvement 


\section{Capítulo 1}

\section{INTRODUÇÃO}

Com a descoberta de Hawking de rue buracos negros evaporam devido a efeitos quânticos [1], houve uma grande quantidade de debates sobre as propriedades dos buracos negros. Estas discussöes tratam das possibilidades de incluir efeitos quânticos na teoria de gravitaçẽo, embora em 4d não exista uma teoria quântica para a gravitaçăo.

A descoberta da evaporação de Hawking levanicy um problema sério cuja soluçăo requer um melhor entendimeno da física na escala de Planck. Ëste é o problema relacionado com a perda de informaçâto. Tratando este problema de forma abreviada, consideramos um buraco negro formado a partir de um estado puro |⿰㇒⿻二丨冂 $>$. Este buraco negro entäo evapora e de acordo com o cálculo de Hawking o estado final é um estado tớmico. Isto significa que a matriz densidade é da forma $\sum_{\alpha} p_{\alpha}\left|\psi_{\alpha}><\psi_{\alpha_{\alpha}}\right|$, onde $\mid \psi_{\alpha}>$ săo estados base normalizados e $p_{n}$ números, dos quais mais de um ê diferente de zero. Neste caso os $p_{a}^{\prime} s$ são aproximadamente as probabilidades térmicas usuais. De acordo com o cálculo de Hawking se o buraco negro näo deixa um "rennant" isto significa que na teoria quântica de buracos negros estados puros podem evoluir para estados térmicos. Isto está em conflito com as leis usuais da mecảnica quântica que senpro preservan a evoluçăo unitária dos estados. Comparando estados puros e térmicos, verificanos que existe perda de informação de fase nestes tultimos. Uma medida útil da perda de informação é dada pela entropia $S=-\sum_{a} p_{a} \ln p_{a}[2][3]$.

Hawking propôs subsequentemente ao seu trabalho a modificação da mecânica quântica para incluir o problema de perda de informą̧̧äo. Entretanto, criar uma dinâmica aiternativa é problemático. Nesta situação o melhor que se pode fazer é estudar modelos 
simplificados que incluem o colapso gravitacional.

Nos últîmos anos uma compreensão mais avançada da evaporação do buraco negro foi adquirida através da investigação de modelos simplificados en duas dimensões. 0 modelo sirnplicado mais realístico estudado até o momento é o do campo escalar esfericamente simétrico em $4 d[4]$. Entretanto, a teoria de cumpos em $2 \mathrm{~d}$ obtida é ainda muito complicada para ser útil, por isto é necessário necorrar a modelos mais simples. Vários modelos mais simples foram estudados recentementa incluindo o colapso de uma camada de poeira [5], colapso de uma nuvem de poeira esférica [f] a mais nolavelnonte as teotias de gravitação kilatôntcas am a 2 [3][7][8][9] [10][11].

Os modelos dilatônicos säo obtidos a partir do limite de baixa energia da teoria de cordas com supersimetria quebrada [8]. Fm particular, o modelo de CGHS (Callan, Giddings, Harvey e Strominger) para a gravitaçäo dilatônica [2] atraiu uma grande atençăo, já que é classicamente solúvel e a solução descreve a formaçăo de um buraco negro em 2d quando acoplamos $\mathrm{N}$ campos de matéria à gravitaç艹o. Alén disso o modelo assim obtido apresenta evaporaçăo de Hawking e é uma teoria de campos lenormatizável. A

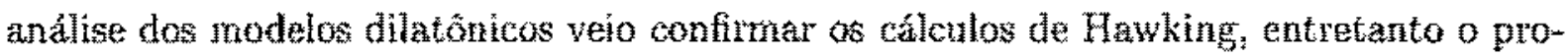
blema da perda de informagăo levou os pesulisadores a procurar axplicacōes alternativas,

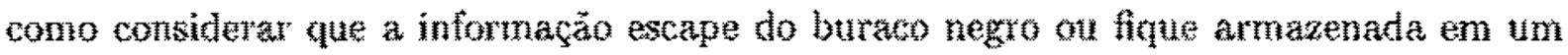
"vemnant" deste. Ambas as altemativas apresentam diffuldades conforme descreveremos a seguir e portanto o problema permanece insoltivel.

Discutitemos agora as alternativas mencionadas acima usando os resultados encontrados na literalura para o modelo de CGHS acoplado à campos de matéria e, en alguns casos, variacöes deste, como por exemplo, o modelo de RST (Russo, Susskind e Thorlacius) [12]. O modelo de CGHS permitiu confirmar as previsões de Hawking sobre a evaporacto do buraco negro. Entretanto, neste caso, tanto a entropia total quanto a energia da matrit densidade säo infinitos. Isso se deve no fato de que no cálculo de emissão de Hawking nẫo se leva en consideração o efeito da radiaçăo sobre a geometria do buraco negro durante a evaporaçăo. Este efeito conheldo como "backreaction" e pode ser induido no modelo fazendo com que o tensor momentomentgia atue como fonte nas equacốs de movimento clássicas para a métrica Extas equaçöes modificadas podem ser obtidas a patil da aço de Polpakov-Liouville [2][3]. A teoria obtida tratada dentro da aproximaçăo semíclássica. O interessante modelo proposto por Russo, Susskind e 
Thorlacius (RST) incorpora todas as caracteristicas da evaporação de Hawking e inclui o efeito de "backreaction". Além disso pode ser resolvido exatamente sem a restrição de $\mathrm{N}$ grande. Entretanto a aproximação semiclássica thăo é válida na fase final da evaporação do buraco negro. Strominger [10] também mostra que o modelo de RST viola o princípio de conservaçăo da energia.

A possibilidade proposta por Hawking de que o buraco negro desapareça no final do processo de evaporaçäo e que a informação seja simplesmente perdida, é na opiniäo de alguns autores, muito radical pois implica em falha da mecànica quântica. Pior que isso, temos que a perda de informação viola o princípio da conservação de energía. A explicação heuristica para isto e a de que a transmissäo do informaçầ requer energia portanto uma perda de informaçấo implita em não conservaşẩo da energin.

A segunda possibilidade é de que a informaçăo seja retornada na radiaçăo de Hawking. A anălise do modelo de CGHS com campos de matéria acoplados à gravitaçăo mostra que o retorno de informaçäo viola os princípios de localidade/causalidade [3]|7]. Este modelo foi estudado utilizando-se uma descrição semiclássica que parece ser vắlida até o buraco negro atingir a massa de Planck $\left(m_{p t}\right)$. Assim, outra possibilidade é de que a informação seja irradiada depots que o buraco negro atinja $M \sim m_{y}$. Aqui o principio de causalidade ordinário não mais se aplica ao interior do buraco negro e fo plausivel que a informaçă escape. Entretanto, neste caso, a única maneira segundo a qual a radiação possa conter tamanha quantidade de informaçăo com uma energia tâo pequena é a de que ela seja constituída de um número muito grande de partículas "soft", por exemplo fótons. Esta hipótese implica, segundo os cálculos, em um tempo de vida do buraco negro da ordem do tempo de vida do universo. Isto implica en uma terceira possibilidade, a de que depois de cessada a radiação de Hawking os buracos negros deixem um "remnant" de longa vida ou estavel.

Se as "temnants", sejam eles estáveis ou de longa vida, resolvem o problema da informaçăo, então deve existir um número infinito de espëcies de "remnants" (um número infinito de estados internos) para armazenar a informaçăo de um buraco negro inicial muito grande. Isto implicaria na produçāo de um número infinito de pares se raciocinarmos usando o conceito da produção de pares de Schwinger [13]. A produçäo de pares de Schwinger é ilustrada em [3] como exemplo para o caso de um "remnant." carregado elétricamente, e vernos que a taxa total de produçăo de pares tende a infinito para um 
rúmero infinito de estados internos.

A tabela abaixo apresenta de forma resumida as propostas para o problema da perda de informaça e os respectivos principios violados

\begin{tabular}{|c|c|}
\hline Proposta & Printipios Violados \\
\hline $\begin{array}{l}\text { Perda de } \\
\text { Informaçào }\end{array}$ & $\begin{array}{c}\text { Unitariedade, } \\
\text { Conservagăo da Energia }\end{array}$ \\
\hline $\begin{array}{l}\text { Informaço } \\
\text { Irradiada }\end{array}$ & $\begin{array}{l}\text {-Durante Colapso/Evaporaçäo: } \\
\text { Localidade/Cautalidade } \\
\text {-Depois de ahingir a Escala de Planck: } \\
\text { Taxa Infinita de } \\
\text { Produça de Pares }\end{array}$ \\
\hline $\begin{array}{l}\text { Intormata Reatida } \\
\text { nos "Remnants" }\end{array}$ & $\begin{array}{l}\text { Taxa Infintua de } \\
\text { Produça de Pares }\end{array}$ \\
\hline
\end{tabular}

Concluimse do exposto acima xue antes do ponto final de evaporaça os modelos em $2 d$ se comportam justamente como Hawking previu para os buracos negros am $4 d$. Os problemas que surgem depois desse ponto ainda precisam ser examinados através de estudos de modelos na escala de Planck.

Neste trabalho, analisamos o modelo de CGHS em 2d utilizando o formalismo ha miltoniano quantizamos a teoria utilizando o método BRST-BFV, desconsiderando o acoplamento com campos de matéria. Esta é uma primeira aproximadào para entender a quantianăa das teorias dilatônicas em 20 . A quantizaçăo do modelo do CGHS utilizando o procedimento de BRST-BFV representa uma soluga nova nâa disponivel na literatura. Fn uma etapa futura poderenos estudar a quantiatazo do modelo acoplando campos de matéria ao campo gravitacional, o que pemitirá analisar a formaço de buracoz negros o a radiaga

A sequencta wada no trabalho para análise da teoria é descrita a seguix. Escrovemos Inlchamente a acou para o modelo de CGHS na forma ustal. Uthizando uma transformatăo conforme reescrevenos o modelo en uma forma mais simples para suntise. Com o modelo nesta foma aplicamos a teoria de sistemas vinculados a segur atilizamos o 
mét,odo de quantizaçào de BRST-BFV para obter a carga de BRST, a açấo efetiva e a função de partição para o modelo no gauge conforme. Integramos, então, os fantasmas que estão desacoplados dos campos da teoria. Utilizamos em seguida, uma transformação canônica que torna os vinculos quadráticos. Com os vinculos na forma quadrática a funçäo de partiçẫo obtida anteriormente repressenta uma integral gaussitana nos campos. Reeserevemos a carga de BRST e a ação efetiva para o modelo onde a contribuiçăo dos fantasmas é escrita on-shell. Verificamos que o modelo original escrito em funçăo do campo gravitacional e do campo escalar dilatônico, mais contribuiçōes dos fantasmas, é agora descrito por uma açäo de dois campos escalares, um com energia cinética positiva e o outro com energia cinética negativa, mais contribuiçăo dos fantasmas. Realizamos uma segunda transformaçäo canônica nos fantammas que permits escrever os vínculos na forma do tensor momento-energia. Utilizando o procedimento de quantização canönico expandimos os campos e fantasmas em operadores de criaçăo e anicuilaçăo e analisamos a álgebra para o tenșor momento-energía. Verificamos a presença de uma anomalia na álgebra. Esta anomalia năo é anulada considerando a contribuição dos fantasmass. Introduzimos entăo um termo de improvement na ação (termo de superficie) que nāo aitera a dinâmíca do sistema. Reobtemos a Algebra do tensor momento-energia total relativo aus campos. Verificamos que as älgebras, quăntica e clássica, para o tensor monento-tnergia, sơ são de primeira classe na condiçẫo em que as cargas $Q_{a}$ (introduzidas no termo de improvement) satisfazem à condição $Q_{n} Q^{a}$ igual a zero. Nesta condição só conseguimos anular a anomalia quando desprezamos a contribuiçăo dos fantasmas. Discutimos támbém o caso em que $Q_{a} Q^{a}$ é diferente de zero, sem introduzir a definiçāo de parênteses de Dirac para converter víneulos de segunda classe em vínculos de primeira classe. No caso em que $Q_{a} Q^{a}= \pm 13 / 6 \pi$ conseguimos anular a anomalia. Quando $Q_{a} Q^{a}=\mp 1 / 6 \pi$, verificanos, que as possiveis soluęöes possuem norma negativa, o que ê inconsistente com a definiçào usual do espaço de Hilbert físico.

Analisamos também as possiveis escolhas para o vécuo do sistema.

Mostramos ainda, um caso rnais simples de anulamento da anomalia utilizando o procedimento canônico, ou seja, desconsideramos o terno de improvement e o procedimento de BRST-BFV.

Finalmente, utilizamos o método de quantizaçăo de Dirac e obtemos as soluȩões para a funçäo de onda que satisfazem aos vínculos do sistema. Nesta etapa utilizamos também 
um procedimento alternativo ao método de quantização de Dirac conhecido como quantização RPS que facilita a obtençẩo do funcional de onda no caso em que desconsiderarnos o termo de improvement. Utilizamos este método e reobtemos as soluçōes encontradas usando-se o primeiro método. As soluçôes para o funcional de onda no caso em que $Q_{a}$ é diferente zero, na condiçăo em que $Q_{\mathrm{a}} Q^{\mathrm{a}}$ é igual a zero, representam uma contribuiçăa nova na literatura,

Acrescentamos uma discussão sobre o problema de ordenanento normal nos vinculos, mostrando a modificaçăo dos vínculos na forma operatorial para uma prescrição arbitrária do ordenamento dos operadores.

Concluimos, finalmente, que o modelo de CGHS em $2 \mathrm{~d}$ pode ser quantizado utilizandose o procedimento de BRST-BFV. 


\section{Capítulo 2}

\section{MODELO DE CGHS}

\subsection{Descrição do Modelo}

O Modelo de CGHS (Callan, Giddinge, Harvey e Strominger) é descrito pela af̧äo [2]

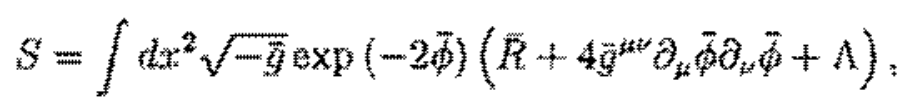

onde $\bar{\phi}$ é um campo escalar chamado campo dilatonico, $\vec{R}$ e o escalar de curvatura construído a partir do tensor métrico $\bar{g}_{\mu w}, \bar{g}$ é o determinante de $\tilde{g}_{*}$ e $\Lambda$ a constante cosmológica.

O escalar de curvatura $\bar{R}$ é definido por $[4]$

$$
\ddot{R}=\tilde{g}^{i k} \tilde{R}_{\mu \nu}
$$

onde $\vec{R}_{\mu u}$ é o tensor de Ricci;

$$
\bar{R}_{p t}=\tilde{R}_{\mu p v}^{\alpha}
$$

$\bar{R}_{\gamma \sigma p}^{\nu}$ é o tensor de curvatura definido por

$$
\bar{R}_{k i m}^{i}=\frac{\partial \bar{\Gamma}_{k m m}^{i}}{\partial x^{l}}-\frac{\partial \bar{\Gamma}_{k l}^{i}}{\partial x^{m}}+\bar{\Gamma}_{n l}^{i} \bar{\Gamma}_{k m}^{n}-\bar{\Gamma}_{n m}^{i} \bar{\Gamma}_{k l}^{n}
$$


Vamos usar uma transformação conforme que permita reescrever o modelo de CGHS sem o termo exponencial [15][16]

$$
\begin{aligned}
\phi & =\exp (-2 \bar{\phi}) \\
g_{\mu \nu} & =\exp (-2 \bar{\phi}) \bar{g}_{\mu \nu}
\end{aligned}
$$

O escalar de curvatura é transformado, a menos de termos de superície, em

$$
\bar{R}=\exp (-2 \bar{\phi}\}\left[R-4(\nabla \bar{\phi})^{2}\right]
$$

e a ação pode ser então escrita na forma simplificada

$$
S=\int d x d l \sqrt{-g}(\phi R+\Lambda)
$$

Analisaremos o modelo de CGHS na forma dada acima.

$\mathrm{Na}$ análise do modelo de CGHS vamos utilizar a parametrização ADM (Arnowitt, Deser e Misner) da métrica [14] [17]

$$
g_{\mu \nu}=\left(\begin{array}{cc}
-N_{1}^{2}+g N_{0}^{2} & g N_{0} \\
g N_{0} & g
\end{array}\right)
$$

Procedendo com o cálculo de $\mathrm{R}$, utilizando a parametrização acima, obtemos

$$
\begin{aligned}
R & =-\left[N_{1} \dot{g}^{2}+2 g \dot{g} \dot{N}_{1}-2 g \ddot{g} N_{1}-2 \dot{g} g^{\prime} N_{0} N_{1}+2 g g^{\prime} N 1 \dot{N}_{0}-2 g g^{\prime} N_{0} \dot{N}_{1}\right. \\
& +g^{\prime 2} N_{0}^{2} N_{1}+4 g \dot{g} N_{1} N_{0}^{\prime}-4 g^{2} \dot{N}_{1} N_{0}^{\prime}-6 g g^{\prime} N_{0} N_{1} N_{0}^{\prime}-4 g^{2} N_{1} N_{0}^{\prime 2} \\
& -2 g \dot{g} N_{0} N_{1}^{\prime}+2 g g^{\prime} N_{0}^{2} N_{1}^{\prime}-2 g^{\prime} N_{1}^{2} N_{1}^{\prime}+4 g^{2} N_{0} N_{0}^{\prime} N_{1}^{\prime}+4 g \dot{g}^{\prime} N_{0} N_{1} \\
& \left.+4 g^{2} N_{1} \dot{N}_{0}^{\prime}-2 g g^{\prime \prime} N_{0}^{2} N_{1}-4 g^{2} N_{0} N_{\mathrm{l}} N_{0}^{\prime \prime}+4 g N_{1}^{2} N_{0}^{\prime \prime}\right] /\left(2 g^{2} N_{1}^{3}\right)
\end{aligned}
$$

A análise do modelo de CGHS será realizada utilizando a teoria de sistemas vinculados descrita na próxima seçāo. 


\subsection{Sistemas vinculados}

A teoria de sistemas vinculados é aqui descrita dentro do formalssmo hamiltoniano [18]. Usando este formalismo partimos inicialmente de uma açăo geral

$$
S=S\left[\varphi_{i}\right]=\int d t L\left(\varphi_{i}, \partial_{a} \varphi_{i}\right)
$$

onde $L$ ê a lagrangeana e $\varphi_{i}\left(x_{p}\right)(i=1, \ldots, N)$ o campos da teoria. Definimos a densidade de lagrangeana $\mathcal{L}$ por

$$
L \equiv \int d^{D-1} x \mathcal{L}
$$

onde $\mathrm{D}$ to número de dimensôes do espaço-tempo.

Os momentos canonicamente conjugados aos campos $\varphi_{i}$ são definidos por

$$
\Pi_{i}=\frac{\partial L}{\partial \dot{\varphi}_{i}}
$$

Pelo princípio da mínima ação, devemos ter $\delta S=0$ [19], o que nos leva às equações de movimento de Euler-Lagrange

$$
\frac{\partial L}{\partial \varphi_{i}}-\partial_{k} \frac{\partial L}{\partial\left(\partial_{j} \varphi_{i}\right)}=0
$$

A densidade de hamiltoniana canônica é definida por

$$
\mathcal{H}_{c}=\Pi_{i} \dot{\phi}_{\hat{j}}-\mathcal{L}
$$

e a hamiltoniana canonica por

$$
H_{\mathrm{c}}=\int d^{D-1} x \mathcal{H}_{\mathfrak{c}}
$$

O principio da mínima açăo nos leva às equações de movimento de Hamilton

$$
\dot{\varphi}_{i}=\frac{\partial H_{c}}{\partial \Pi_{i}}, \quad \dot{\Pi}_{i}=-\frac{\partial H_{c}}{\partial \varphi_{i}}
$$


Podemos escrever para as equaçōes de movimento na forma hamiltoniana

$$
\dot{\varphi}_{i}=\left\{\varphi_{i}, H_{c}\right\}, \dot{\Pi}_{i}=\left\{\Pi_{i}, H_{i}\right\}
$$

onde as chaves representam os parênteses de Poisson [18], denotados no texto por PB's.

Na teoria de sistemas vinculados, usamos o hessiano generalizado para campos

$$
W_{i j}^{\mu \nu}=\frac{\partial^{2} L}{\partial\left(\partial_{\mu} \varphi_{i}\right) \partial\left(\partial_{\nu} \varphi_{j}\right)}
$$

onde $i, j=1, \ldots, N$. Em particular para $\mu=0, \nu=0$

$$
W_{i j}^{00}=\frac{\partial^{2} L_{L}}{\partial \dot{\varphi}_{i} \partial \dot{\varphi}_{j}}=\frac{\partial \Pi_{i}}{\partial \dot{\varphi}_{j}}
$$

Temos un sistema regular quando

$$
\operatorname{de}\left(W_{i j}^{00}\right) \neq 0
$$

Neste caso, todas as velocidades $\hat{\varphi}_{i}$ podem ser expressas en termos dos campos $\varphi_{i}$ e dos momentos $\Pi_{i}$. Caso tenhamos

$$
\operatorname{det}\left(W_{i j}^{\infty 0}\right)=0
$$

teremos $m$ velocidades $\hat{\varphi}_{l}$ que não podem ser expressas em termos dos campos e dos momentos. Neste caso dizemos que a teoria possui $m$ vínculos.

Isolando os momentos relativos às "l" linhas em funçāo das outras variáveis obtemos

$$
\Pi_{i}=h_{l}\left(\varphi_{i}, \Pi_{i}, \partial_{j} \varphi_{i}, \partial_{j} \Pi_{i}\right)
$$

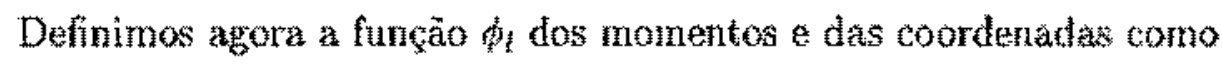

$$
\varphi_{1} \equiv \Pi_{i}-h_{i}\left(p^{i}, \Pi_{k}, \partial_{j} p^{i}, \partial_{j} \Pi_{k}\right)
$$




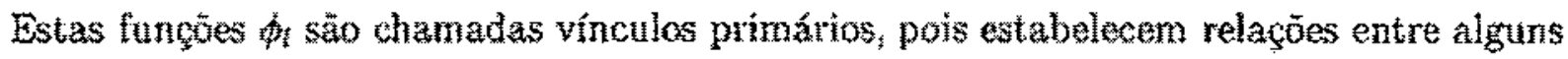
momentos e outras variáveis, definem um subespaço $\Gamma_{\text {; }}$ (no espaço de fase $\Gamma$ ) onde os vínculos säo nulos.

Vamos utilizar a notação introduzida por Dirac de sinal de igualdade fraca" $\approx$ ". Este sinal indica que $\phi_{1}$ só pode ser colocado igual a zero depois de calculatos os parêniteses de Poisson nos cálculos desejados [20]. Assim, escrevemos

$$
\phi_{l} \approx 0(l=1, \ldots, n)
$$

Como os vínculos são nulos a hamiltoniana canônica fica daterminada a menos de fatores cuue săo os multiplicadores de Lagrange

$$
\mathcal{H}_{p}=\mathcal{H}_{c}+\lambda^{l} \dot{\phi}_{l}
$$

onde $\mathcal{H}_{p}$ é a densidade de hamiltoniana primária

As equaçöes de movimento säo agora escritas como

$$
\dot{\varphi}_{i}=\left\{\varphi_{i}, H_{p}\right\} \quad: \dot{\Pi}_{p}=\left\{\Pi_{i}, H_{p}\right\}
$$

ou

$$
\begin{gathered}
\dot{\phi}_{i}(x)=\left\{\varphi_{i}(x), H_{c}\right\}+\int d^{D-1} x^{2} \lambda^{\prime}\left(x^{\prime}\right)\left\{\varphi_{i}(x), \phi_{l}\left(x^{i}\right)\right\} \\
\dot{\Pi}_{i}(x)=\left\{\Pi_{i}(x), H_{c}\right\}+\int d^{D-1} x^{\prime} \lambda^{l}\left(x^{\prime}\right)\left\{\Pi_{i}(x), \phi_{l}\left(x^{\prime}\right)\right\} \\
\phi_{1} \approx 0
\end{gathered}
$$

Devemo impor uma condição sobre os vintulos, a de que des permaneçan fracamente nulos com o decorrer do tempo [18] [20]. Portanto devemos ler

$$
\dot{\phi}(x) \approx 0
$$


ou

$$
\left\{\phi_{l}(x), H_{p}\right\} \approx 0
$$

ou expandindo $H_{p}$

$$
\left\{\phi_{l}\{x), H_{c}\right\}+\int d^{D-1} x^{\prime} \lambda^{k}\left(x^{\prime}\right)\left\{\phi_{1}(x\}, \phi_{k}\left(x^{\prime}\right)\right\} \approx 0
$$

Esta equação possui três tipos de solução

A) $0 \approx 0 ;$ ou seja $\left\{\phi_{t}, H_{p}\right\}=0$. Neste caso temos uma identidade e nazo obtemos nenhuma relação entu as variáveis do espaço de fase.

B) Soluçồes que envolvem os $\lambda^{k}$. Estas soluçoes impöen relaçöes que definem alguns dos multiplicadores de lagrange.

C) Soluçōes que näo envolvem os $\lambda^{k}$. Essse tipo de soluçāo estabelece relações extras entre as variáveis do espaço de fase que chamaremos vinculos secundários, representados por

$$
\phi^{22} \approx 0
$$

onde $r=1, \ldots, u$

Os vínculos secundários devem também permanecer fracamente nulos com o decorrer do tempo, e procedendo dessa forma poderemos ainda obter mais relaçöes que determinem outros multiplicadores de lagrange ou que forneçan mais vínculos. Este procedimento deve ser repetido até obtermos apenas soluçöes do tipo A.

Obtidos todos os vínculos da teoria podemos definir a densidade de hamiltoniana estendida

$$
\mathcal{H}_{e}=\mathcal{H}_{0}+\lambda^{s} \phi_{s}
$$

onde $s=1, \ldots, v(v$ é o numero total de vínculos) 
A evoluçăo temporal de uma função qualquer do espaço de fase pode ser obtida tanto de $H_{c}, H_{p}$ ou $H_{e}[18]$

$$
\dot{A}=\left\{A_{1} H_{i}\right\} \approx\left\{A, H_{p}\right\} \approx\left\{A_{q} H_{\mathrm{E}}\right\}
$$

Agora definimos outra dassificafão importante para os vínculos. Säo chamados vínculos de primeira classe os vínculos que possuem parenteses de Poisson fracamente nulos com todos os outros vínculos da teoria, e vínculos de segunda classe os que não possuem esta propriedade. Represtentamos os vínculos de segunda classe por $x_{k}(k=1, \ldots, t)$. Consideramos agora $\phi_{i}(i=1, \ldots v-t)$ representando os vineulos de primeitra classe. Assim

$$
\left\{\hat{\phi}_{i}, \phi_{2}\right\}=\left\{\phi_{i}, x_{i}\right\} \approx 0
$$

$$
\left\{\chi_{k}, \chi_{l}\right\} \neq 0
$$

onde $=1, \ldots, z-t$ e $k, l=1, \ldots \ldots, t$.

Representamos os paranteses de Poisson dos vinculas de segunda classe como uma

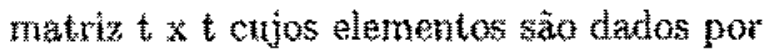

$$
\begin{aligned}
& \left\{\chi_{k}, \chi_{l}\right\}=M_{k l} \\
& \operatorname{det}\left(M_{k l}\right) \neq 0
\end{aligned}
$$

Caso o determinante da matriz $\left(M_{4}\right)$ seja zaro, teremos combinacoes linerares de vinculos de segunda classe que serâo vínculos de prineira classe uma vez que depois de escalonada a matriz apresenta algumas linhas nulas.

Utilizando esta notaçă a densidade de hamiltoniana estendida pode ser escrita como

$$
H_{\hat{x}}=\mathcal{H}_{\mathrm{r}}+\lambda^{i} \phi_{i}+\alpha^{k} \chi_{k}
$$

onde $=1, \ldots, v-\frac{1}{2} h=1, \ldots$,

A preservaçào dos vínculos de primeira classe no ternpo implica en 


$$
\dot{\phi}_{i} \approx 0
$$

ou

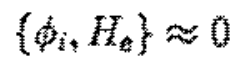

o que resulta em

$$
\left\{\phi_{i}, H_{c}\right\} \approx 0
$$

e para os vinculos de segunda classe

$$
\dot{x}(x) \approx 0
$$

ou

$$
\left\{x_{k}(x), H_{e}\right\} \approx 0
$$

cexpandindo $H_{e}$

$$
\left\{\chi_{k}(x), H_{e}\right\}+\int d^{D-1} x^{\prime} \alpha^{j}\left(x^{\prime}\right)\left(\chi_{k}(x), \chi_{j}\left(x^{\prime}\right)\right\} \approx 0
$$

ou ern termos da matriz $\left(M_{k j}\right)$

$$
\left\{\chi_{k}(x), H_{c}\right\}+\int d^{D-1} x^{t} \alpha^{\xi}\left(x^{t}\right) M_{k j}\left(x, x^{z}\right) \approx 0
$$

A equaçăo de movimento para uma funçăo "A" das variáveis do espaço de fase ê dada neste caso por

$\dot{A}(x)=\frac{\partial A(x)}{\partial t}+\left\{A(x), H_{n}\right\}+\int d^{D-1} x^{\prime} \lambda^{i}\left(x^{\prime}\right)\left\{A(x), \phi_{i}\left(x^{t}\right)\right\}+\int d^{D-i} x^{t} \alpha^{k}\left(x^{t}\right)\left\{A(x), x_{k}\left(x^{t}\right)\right\}$

ou substituindo a expressäo para $\alpha^{k}$ 


$$
\begin{aligned}
\dot{A}(x) & \approx \frac{\partial A(x)}{\partial t}+\left\{A(x), H_{c}\right\}+\int d^{D} x^{\prime} \lambda^{i}\left(x^{3}\right)\left\{A(x), \phi_{i}\left(x^{\prime}\right)\right\} \\
& -\iint d^{D+1} x^{t} d^{D-1} y\left\{A(x), \chi^{j}\left(x^{j}\right)\right\} M_{k j}^{-1}\left\{x^{t}, y\right)\left\{x^{j}\left(y^{\prime}\right), H_{c}\right\}
\end{aligned}
$$

Introduzindo os parênteses de Dirac definidos por [20]

$$
\{A(x), B(y)\}_{D}=\{A(x), B(y)\}-\iint d^{D-1} x^{t} d^{D-1} y^{t}\left\{A(x), \chi^{i}\left(x^{k}\right)\right\} M_{j k}^{-1}\left(x^{t}, y^{h}\right)\left\{x^{k}\left(y^{\prime}\right), B(y)\right\}
$$

escrevemos

$$
\dot{A}(x) \approx \frac{\partial A(x)}{\partial t}+\left\{A(x), H_{c}\right\}_{D}+\int d^{D-1} x^{\prime} \lambda^{i}\left(x^{3}\right)\left\{A(x), \phi_{i}\left(x^{3}\right)\right\}
$$

Na próxima seção aplicamos a tereria de sistemas vinculados ao modelo de CGHS. 


\subsection{Teoria de Sistemas Vinculados Aplicada ao Mo- delo de CGHS}

A partir da acâo dada pela equacio (2.7) definimos os momentos canonicamente conjugados a g e a respectivamente por

$$
p=\frac{\partial L}{\partial \dot{g}}, \Pi_{\phi}=\frac{\partial L}{\partial \dot{\phi}}
$$

usando as expressöes para os momentos juntamente com a expressäo para $R$ (2.9) na equaçäo para a açäo (2.7) obtemos para a hamiltoniana do modelo de CGHS [14][21]

$$
H_{c}=\int d x\left(\frac{N_{1}}{\sqrt{g}} G_{0}+N_{0} G_{1}\right)
$$

onde $G_{0} G_{1}$ säo dados por

$$
\begin{aligned}
& G_{0}=-g p \Pi_{\hat{\psi}}-g \Lambda+2 \phi^{\prime}-\frac{\phi^{\prime} g^{\prime}}{g} \\
& G_{1}=\Pi_{\phi^{\prime}} \phi^{\prime}-2 p^{\prime} g-p g^{\prime}
\end{aligned}
$$

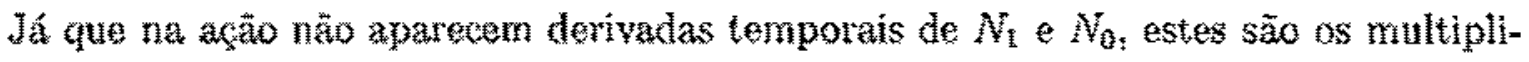

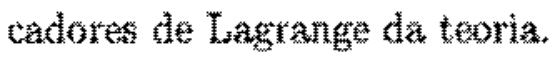

Introduindo o fator conforme $p$

$$
g=e^{p}
$$

o momento p a transormado da seguinte forma

$$
p=e^{-p} \Pi_{p}
$$

e os vínculos passam a ser dados por 


$$
\begin{aligned}
& G_{0}=-\Pi_{\rho} \Pi_{\phi}-\Lambda \exp ^{\rho}+2 \phi^{\prime \prime}-\rho^{\prime} \phi^{\prime} \\
& G_{1}=\Pi_{\phi} \phi^{t}-\Pi_{p} \rho^{t}-2 \Pi_{p}^{\prime}
\end{aligned}
$$

$G_{0}$ e $G_{1}$ sấo na realidade os vínculos secundários da teoria jă que $N_{0}$ e $N_{1}$ săo multiplicadores de Lagrange. Os vínculos primários são dados pelos momentos associados a $N_{0}$ e $N_{1}$, respectivamente dados por $P_{N_{0}}$ e $P_{N_{1}}$.

Assim a hamiltoniana primária da teoria $(2.25)$ é a própria hamiltoniana canônica e a evolução temporal de $G_{0}$ e de $G_{1}$

$$
\begin{aligned}
& \dot{G}_{1}=\left\{G_{1}, H_{c}\right\} \approx 0 \\
& \dot{G}_{0}=\left\{G_{0}, H_{c}\right\} \approx 0
\end{aligned}
$$

não leva à novos vínculos securtitários pois obtemos a solucao $0 \approx 0$.

Os vinculos da teoria são de prineira classe uma vez que satidatam a álgebra [22] [23]

$$
\begin{aligned}
& \left\{G_{0}(x), G_{0}(y)\right\}=\delta^{\prime}(x-y)\left[G_{1}(x)+G_{1}(y)\right] \\
& \left\{G_{1}(x), G_{0}(y)\right\}=\delta^{\prime}(x-y)\left[G_{0}(x)+G_{0}(y)\right] \\
& \left\{G_{1}(x), G_{1}(y)\right\}=\delta^{z}(x-y)\left[G_{1}(x)+G_{1}(y)\right]
\end{aligned}
$$

onde $\delta^{\prime}$ corresponde $a \frac{\partial j}{\partial x}$. 


\section{Capítulo 3}

\section{QUANTIZAÇÃO DO MODELO DE CGHS}

Vamos proceder com a quantização do modelo de CGHS utilizando o método BRSTBFV proposto por Batalin, Fradkin e Vilkovisky [24][25][26] [27]. Na próxima secä destrevemos o método BRST-BFV e na seçăo 3.2 aplicamos o método an modelo de COHS.

\subsection{Método de Quantização BRST-BFV}

Nesta seçäo vamos tratar da quantizaçāo BRST (Bechi, Rouet a Stora)[24][25] de um sistema dentro do formalismo hamiltoniano desenvolvido por Batalin, Fradkín e Vilkovisky, conhecido como BRST-BFV [26] [27].

Vamos considerar um sistema definido em um espaço de fase descrito por campos $\varphi_{i}$ cujos momentos associados sảo dados por $\Pi_{i}$. Eliminamos os vínctulos de segunda classe utilizando o procedimento de Dirac, e obtemos a relaçōes de comutaçăo básicas

$$
\begin{aligned}
\left\{\psi_{i}(x), \Pi_{j}(y)\right\}_{D} & =\alpha_{i j} \delta(x-y) \\
\left\{\varphi_{i}(x), \varphi_{j}(y)\right\}_{D} & =0
\end{aligned}
$$

onde $\alpha_{i_{z}}$ s sä0 constantes. 
Vamos considerar nesta seçắ os parênteses de Dinte representados de forma itêtictu aos parenteses de Poisson.

Os vínculos deste sistema sầ representados por $\phi_{i}\left(\varphi_{i}, \Pi_{j}\right)$, säo de primeira classe e satisfazem a algebra

$$
\left\{\phi_{i}(x), \phi_{j}(y)\right\}=\int d^{D \cdots k} z C_{i j}^{k}(x, y, z) \phi_{k}(z)
$$

onde $C_{4}^{k}(x, y, z)$ a estendida para este sistema

$$
H_{\varepsilon}=\int d^{D-1} y\left(H_{\varepsilon}+\lambda_{i}\right)
$$

onde $\lambda_{i}$ são os multiplicadores de Lagrange dos vinculos, ou seja funções indeteminadas das coordenadas.

No método de quantízação BFV, o espaço de fase é estendido de forma a considerar os multiplicadores de Lagrange $\lambda_{i}$ como variáveis dinâmicas e associar aos nesmos momentos $p_{i}$ de lomma que

$$
\left\{\lambda^{3}(x), p_{j}(y)\right\}=\delta_{j} \partial(x-y)
$$

Como năo podemos modificar o conteúdo dinámico da teoria estes momentos stấo impostos como noús vínculos

$$
p_{i}=0
$$

Com esta consideraça inclumos novas constantes de estrutura também representadas por $C_{j i}^{i}$.

A etapa seguinte no nétodo de BPV consiste en introduzir um par de fantasmas canônicamante conjugudo para cada víneulo e com partade de Grassmann [28] oposta a do vinculo considerado. Para os vínculos usaais da leoria usamos o par de fantasmats canônicamente conjugato $\left(\eta, p_{i}\right)$ e para os vinculos associados aos multiplicatore de Lagrange o par $\left(\eta^{i}, p_{i}\right)$. Estes fantasmas obedecem, por definicho. 


$$
\begin{aligned}
& \left\{\eta^{i}{ }_{i}^{j}\right\}=0 \\
& \left\{\tilde{p}_{i}, \tilde{P}_{j}\right\}=0 \\
& \left\{\eta^{\hat{i}}, \overrightarrow{\mathcal{P}}_{j}\right\}=-\mathrm{g}_{j} z_{j}(x-y) \\
& \left\{\vec{\eta}^{\hat{i}}, \tilde{\eta}^{j}\right\}=0 \\
& \left\{p_{i}, p_{j}\right\}=0 \\
& \left\{\bar{\eta}^{i}, P_{j}\right\}=-\delta_{j}^{j} \partial(x-y) \\
& \left\{\bar{n}^{i}, \eta^{j}\right\}=0 \\
& \left\{\bar{p}_{i} p_{i}\right\}=0
\end{aligned}
$$

É conveniente, neste ponto, introduzimos o conceito de número de fantasmas. A cada urna das variảveis do espaço de fase estendido (incluindo os multiplicadores de Lagrange e os fantasmas) associaremos um número de fantasmas representado por "gh" [20]

$$
\begin{aligned}
& g h\left(\varphi^{j}\right)=g h\left(\Pi_{i}\right)=0 \\
& g h\left(\phi^{j}\right)=g h\left(\lambda^{j}\right)=0 \\
& g h\left(\eta^{\hat{i}}\right)=g h\left(p^{j}\right)=1 \\
& g h\left(\bar{p}_{j}\right)=g h\left(\eta_{i}\right)=-1
\end{aligned}
$$

O número de fantasmas respeita a seguinte propriedade

$$
g h(A B)=g h(A)+g h(B)
$$

A etapa seguinte do método BFV é escrever a carga de BRST que é dada pela seguinte expressãa [26][27]

$$
\begin{aligned}
Q & =\int d^{D-1} x\left(\eta^{i}(x) \phi_{i}(x)+p^{i}(x) \mathcal{P}_{i}(x)\right) \\
& -\frac{1}{2}(-1)^{x_{i}+n_{j}} \int d^{D-1} x d^{D-1} y d^{D-1} z \mathcal{P}_{i}(z) C_{j k}^{i}(x, y, z) \eta^{k}(y) \eta^{j}(x)+\ldots
\end{aligned}
$$


onde $p_{i}$ 's säo os momentos associados aos multiplicadores de Lagrange e $n_{i}$ definido como a paridade de Grassmann da funçäo $\phi_{i}$ (vínculo).

A carga de BRST é nilpotente ou seja

$$
\{Q, Q\}=0
$$

e possui número de fantasmas igual a 1.

Na etapa final do método BFV, escrevemos a funçāo de partígão

$$
Z=\int \mathcal{D} \varphi^{i} \mathcal{D} \Pi_{i} D \lambda_{j} \mathcal{D} p^{j} \mathcal{D} \eta^{j} \mathcal{D} \bar{P}_{j} D \eta^{i} \mathcal{D} p_{j} e^{i S_{e s}}
$$

onde $S_{e f}$ é a açăo efetiva dada por [26][28]

$$
S_{e f}=\int d t\left[\int d^{D-1} x\left(\Pi^{i} \dot{\phi}_{i}+p^{j} \dot{\lambda}_{j}+\hat{\eta}^{i} \overline{\mathcal{P}}_{i}+\dot{\eta}^{j} \mathcal{P}_{i}-H_{c}\right) \ldots\left\{Q_{i} \Psi\right\}\right]
$$

e onde 4 é uma função Grasmaniana das variáveis do espaço de fase chamada função fixadora de gauge. $\Psi$ em geral é dada por [29]

$$
\Psi=\int d^{D-1} x\left(\vec{\eta}^{i} x_{i}+\overrightarrow{\mathcal{P}}_{i} \lambda^{i}\right)
$$

onde $\chi_{i}$ săo funçöes arbitrárias dos campos conhecidas como funçöes fixadoras de gauge.

É interessante observar que ação efetiva é construída de forma a ser invariante sọb transformaçōes geradas pelo gerador Q.

O teorema de Batalin, Fradkin e Vilkovisky [26] [27] garante que a funçäo de partição $Z$ dada por (3.11) é independente da funçâo fermiônica arbityária $\Psi$.

Este é básicamente o procedimento de quantizaçăo BRST-BFV. Na próxima seção aplicamos o método BFV ao modelo de CGHS. 


\subsection{Quantização BRST-BFV do Modelo de CGHS}

O modelo de CGHS será aqui analisado utilizando-se a açăo escrita na forma apresentada na equaçẵo (2.7)

$$
S=\int d x d \sqrt{-g}(\phi R+\Lambda)
$$

Seguindo o procedimento de quantizaçăo BRST-BFV descrito na secă anterior, identiftcamos iniciamente todos os vinculos da teoria com os respectivos pares de fantasmas. Obtemos

$$
\begin{gathered}
G_{0} \rightarrow\left(\eta^{0}, \bar{P}_{0}\right) \\
G_{1} \rightarrow\left(\eta^{1}, \bar{P}_{1}\right) \\
P_{N_{0}} \rightarrow\left(\tilde{\eta}^{1}, P_{1}\right) \\
P_{N_{1}} \rightarrow\left(\eta^{0}, P_{0}\right)
\end{gathered}
$$

onde todos os vínculos sẫo bosônicos e. portanto, possuem paridade zero, e os fantasmas variáveis fermiônicas possuindo paridade 1.

Os fantasmas respeitam os seguintes PB's básicos

$$
\begin{aligned}
\left\{\eta^{i}, \eta^{j}\right\} & =0 \\
\left\{\ddot{p}_{i}, \ddot{p}_{j}\right\} & =0 \\
\left\{\eta^{i}, \ddot{p}_{j}\right\} & =-\delta_{j}^{i} \delta(x-y) \\
\left\{\tilde{\eta}^{i}, \ddot{\eta}^{j}\right\} & =0 \\
\left\{p_{i}, \mathcal{P}_{j}\right\} & =0 \\
\left\{\tilde{\eta}^{i}, p_{j}\right\} & =-\delta_{j}^{i} \delta\{x-y\} \\
\left\{\eta^{i}, \eta^{j}\right\} & =0 \\
\left\{\bar{p}_{i}, \mathcal{P}_{j}\right\} & =0
\end{aligned}
$$

Da algebra do vínculos fornecida pelas equaçöes (2.59) obtemos as constantes de estrutura para os vinculos 


$$
\begin{aligned}
& C_{00}^{0}=0 \\
& C_{00}^{1}=\delta^{y}(x-y) \delta(x-z)+\delta^{z}(x-y) \delta(y-z) \\
& C_{10}^{0}=\delta^{y}(x-y) \delta(x-z)+\delta^{\prime}(x-z) \delta(y-z) \\
& C_{10}^{1}=0 \\
& C_{11}^{0}=0 \\
& C_{11}^{1}=\delta^{y}(x-y) \delta(x-z)+\delta^{\prime}(x-y) \delta(y-z)
\end{aligned}
$$

Para facilitar os culculos, redefinimos

$$
N_{1} \rightarrow \frac{N_{1}}{\sqrt{g}}
$$

Portanto $P_{N_{1}}$ representa o momento associado ao novo multiplicador de Lagrange $N_{1}$ Escrevemos agora para a carga de BRST (3.9)

$$
\begin{aligned}
Q & =\int d x\left[\eta^{0} G_{0}+\eta^{1} G_{1}\right]+\int d x\left[P_{1} P_{N_{0}}+P_{0} P_{N_{1}}\right] \\
& +\int d x\left[\overline{\mathcal{P}}_{0} \eta^{1} \eta^{0 t}+\bar{P}_{0} \eta^{0} \eta^{1}\right]+\int d x\left[\tilde{P}_{1} \eta^{0} \eta^{0 t}+\bar{P}_{1} \eta^{1} \eta^{1}\right]
\end{aligned}
$$

Pode-se verificar que $Q$ é nilpotente. As variaçōes de BRST geradas por $\mathrm{Q}$ sao definidas para um campo arbitrário "A" por

$$
A=\{A, Q\}
$$

Para o modelo de CGHS as variaçóes dos campos são dadas por (para os cálculos envolvendo PB's com variáveis fermiônicas veja apêndice A)

$$
\begin{aligned}
& \delta p=-\frac{\eta^{0} \Pi_{\rho}}{2}+\eta^{3} \rho^{\prime}+\eta^{1} \\
& \delta \phi=-\frac{\eta^{0} \Pi_{p}}{2}+\eta^{1} \phi^{\prime}
\end{aligned}
$$




$$
\begin{aligned}
& \delta \Pi_{j}=-2 \eta^{0 t} \phi^{t}-2 \eta^{0} \phi^{\prime \prime}+\eta^{1 t} \Pi_{p}+\eta^{\mathrm{t}} \Pi_{p}^{\prime}+2 A \eta^{0} e^{3 p} \\
& \delta \Pi_{\phi}=-2 \eta^{0 \prime \prime}-2 \eta^{0} \dot{\rho}^{t}-2 \eta^{0} \rho^{\prime \prime}+\eta^{\mathrm{t} t} \Pi_{\phi}+\eta^{1} \Pi_{\phi}^{s} \\
& \dot{m} \eta^{0}=\eta^{1 t} \eta^{0}+\eta^{0 \prime} \eta^{1} \\
& \delta \eta^{1}=\eta^{0 \%} \eta^{0}+\eta_{z^{1}} \eta^{1} \\
& \delta \tilde{P}_{0}=-G^{0}+\overline{\mathcal{P}}_{0}^{\prime} \eta^{1}+2 \tilde{P}_{0} \eta^{1 t}+\tilde{\mathcal{P}}_{1}^{t} \eta^{0}+2 \tilde{P}_{1} \eta^{0 t} \\
& \delta \overline{\mathcal{P}}_{1}=-G^{1}+\overline{\mathcal{P}}_{0}^{\prime} \eta^{\theta}+2 \overline{\mathcal{P}}_{0} \eta^{0 r}+\overline{\mathcal{P}}_{1}^{\prime} \eta^{1}+2 \overline{\mathcal{P}}_{1} \eta^{1 \prime} \\
& \vec{b} \vec{\eta}^{0}=-P_{N_{1}} \\
& \delta \bar{\eta}^{\mathrm{t}}=-P_{N_{0}} \\
& \delta \mathcal{P}_{0}=\delta \mathcal{P}_{1}=\delta P_{N_{0}}=\delta P_{N_{2}}=0 \\
& \delta N_{0}=P_{1} \\
& \delta N_{1}=P_{0}
\end{aligned}
$$

De (3.13) obtemos para a função fixadorả de gauge

$$
\Psi=\int d x\left[\bar{\eta}^{\mathrm{q}} \chi_{\mathrm{a}}+\bar{\eta}^{\mathrm{t}} \chi_{\mathrm{t}}-\bar{P}_{0} N_{1}+\bar{P}_{1} N_{0}\right]
$$

onde $\chi_{0} \mathrm{e} \chi_{1}$ são funçỏes arbitrárias dos campos e săo utilizadas para fixar o gauge. Agora calcularnos $\{Q, \Psi\}$

$$
\begin{aligned}
& \{Q, \Psi\}=\int d x\left[-P_{N_{1}} \chi_{0}+\bar{\eta}^{0} \delta \chi_{0}-P_{N_{0}} \chi_{1}+\bar{\eta}^{1} \delta \chi_{1}\right. \\
& +G_{0} N_{1}-\overline{\mathcal{P}}_{0}^{\prime} \eta^{2} N_{1}-2 \bar{P}_{0} \eta^{1 /} N_{1}-\overline{\mathcal{P}}_{1}^{\prime} \eta^{0} N_{1} \\
& -2 \tilde{P}_{1} \eta^{0} N_{1}-\bar{P}_{0} \mathcal{P}_{0}-G_{1} N_{0}+\overline{\mathcal{P}}_{0}^{i} \eta^{0} N_{0} \\
& \left.+2 \overline{\mathcal{P}}_{0} \eta^{0} N_{0}+\overline{\mathcal{P}}_{1}^{\prime} \eta^{1} N_{0}+2 \overline{\mathcal{P}}_{1} \eta^{1+} N_{0}+\overline{\mathcal{P}}_{1} \mathcal{P}_{1}\right]
\end{aligned}
$$

Utilizando esta expressăo podemos escrever para a açào efetiva do modelo de CGHS 


$$
\begin{aligned}
& S_{e f}=\int d r \int d w\left[P_{N_{0}} \dot{N}_{0}+P_{N_{1}} \dot{N}_{1}+\eta^{\circ} \bar{P}_{0}+\dot{\eta}^{1} \bar{P}_{1}\right. \\
& +\dot{\bar{\eta}}^{0} \mathcal{P}_{0}+\dot{\eta}^{1} p_{1}+\Pi_{\rho} \dot{\rho}+\Pi_{\phi} \dot{\phi} \\
& -\left\{P_{N_{1} \chi_{0}}+\tilde{\eta}^{0} \delta \chi_{0}-P_{N_{0}} \chi_{1}+\bar{\eta}^{1} \delta \chi_{1}\right. \\
& +G_{0} N_{1}-\overline{\mathcal{P}}_{0}^{\prime} \eta^{\mathrm{l}} N_{1}-2 \overline{\mathcal{P}}_{0} \eta^{\mathrm{v}} N_{1}-\overline{\mathcal{P}}_{1}^{\prime} \eta^{0} N_{1} \\
& \text { - } 2 \overline{\mathcal{P}}_{1} \eta^{0} N_{1}-\overline{\mathcal{P}}_{0} \mathcal{P}_{0}-G_{1} N_{0}+\overline{\mathcal{P}}_{0}^{\prime} \eta^{0} N_{0} \\
& \left.\left.+2 \ddot{\mathcal{P}}_{0} \eta^{0 \prime} N_{0}+\overline{\mathcal{P}}_{1}^{t} \eta^{i} N_{0}+2 \overline{\mathcal{P}}_{1} \eta^{1 *} N_{0}+\overrightarrow{\mathcal{P}}_{1} \mathcal{P}_{1}\right\}\right]
\end{aligned}
$$

e de acordo com (3.11) podemos escrever para a funçāo de partiçāo 2

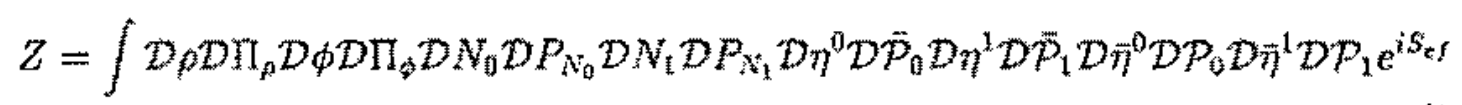

\subsection{Função de Partição para o Modelo de CGHS no Gauge Conforme}

$O$ gauge conforme e definido fixando-se os multiplicadores de Lagrange

$$
\begin{aligned}
& N_{0}=0 \\
& N_{1}=1
\end{aligned}
$$

Antes de fixar o gauge através da escolha de $\chi_{0}$ e $\chi_{\mathrm{I}}$ em (3.21), vamos usar a seguinte transformação na aça efetiva

$$
\begin{aligned}
P_{N_{0}} & \rightarrow \epsilon P_{N_{0}} \\
P_{N_{1}} & \rightarrow \epsilon P_{N_{1}} \\
\tilde{\eta}^{1} & \rightarrow \epsilon \eta^{1} \\
\tilde{\eta}^{0} & \rightarrow \epsilon \eta^{0}
\end{aligned}
$$


E importante observar que esta transformação possui jacobiano igual a 1. Assim obtemos

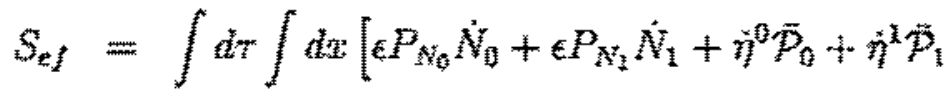

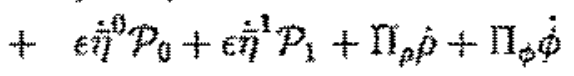

$$
\begin{aligned}
& -\left\{\epsilon P_{N_{1} X_{0}}+\epsilon \eta^{0} \delta_{X_{0}}-\epsilon P_{N_{0} X_{1}}+\epsilon \bar{\eta}^{1} \delta_{X 1}\right. \\
& +G_{0} N_{1}-\overline{\mathcal{P}}_{0}^{\prime} \eta^{1} N_{1}-2 \overline{\mathcal{P}}_{0} \eta^{1 /} N_{1}-\overline{\mathcal{P}}_{1}^{\prime} \eta^{0} N_{1} \\
& -2 \bar{P}_{1} \eta^{0 t} N_{1}-\bar{P}_{0} P_{0}-G_{1} N_{0}+\overline{\mathcal{P}}_{0}^{i} \eta^{0} N_{0} \\
& \left.\left.+2 \overline{\mathcal{P}}_{0} \eta^{\prime \prime \prime} N_{0}^{\prime}+\overline{\mathcal{P}}_{1}^{\prime} \eta^{1} N_{0}+2 \overline{\mathcal{P}}_{1} \eta^{1 \prime} N_{0}+\overline{\mathcal{P}}_{1} \mathcal{P}_{1}\right\}\right]
\end{aligned}
$$

Agora escolhemos para as funçöes fixadoras de gauge

$$
\begin{aligned}
& \chi_{0}=\frac{N_{0}}{\epsilon} \\
& \chi_{1}=\frac{N_{1}-1}{\epsilon}
\end{aligned}
$$

Usando (3.20)

$$
\begin{aligned}
& \delta_{X_{0}}=\frac{P_{1}}{\xi} \\
& \delta_{X_{1}}=\frac{P_{0}}{\epsilon}
\end{aligned}
$$

Agora tomando o limite $\epsilon \rightarrow 0$

$$
\begin{aligned}
& S_{e f}=\int d r \int d x\left[\eta^{0} \tilde{P}_{0}+\dot{\eta} \hat{p}_{1}+\Pi_{\rho} \dot{p}+\Pi_{\dot{q}} \dot{\phi}\right. \\
& \cdots \bar{\eta} P_{1}-\bar{\eta} P_{0}-\left\{P_{N_{1}} N_{0}-P_{N_{0}}\left(N_{1}-1\right)\right. \\
& +G_{01} N_{1}-\overline{\mathcal{P}}_{0}^{\prime} \eta^{\mathrm{l}} N_{1}-2 \overline{\mathcal{P}}_{0} \eta^{1} N_{1}-\overline{\mathcal{P}}_{1}^{\prime} \eta^{0} N_{1} \\
& -2 \tilde{\mathcal{P}}_{1} \eta^{0 t} N_{1}-\overline{\mathcal{P}}_{0} \mathcal{P}_{0}-G_{1} N_{0}+\tilde{\mathcal{P}}_{0}^{\prime} \eta^{\mathrm{a}} N_{0} \\
& \left.\left.+2 \overline{\mathcal{P}}_{0} \eta^{0} N_{0}+\overline{\mathcal{P}}_{1}^{\prime} \eta^{1} N_{0}+2 \overline{\mathcal{P}}_{1} \eta^{1 y} N_{0}+\overline{\mathcal{P}}_{1} \mathcal{P}_{1}\right\}\right]
\end{aligned}
$$


A função de partição é dada por (3.24), com $S_{e f}$ dada por (3.30)

Vamos agora realizar integraçōes em $Z$ sobre os multiplicadores de Lagrange a fantasmas. Integrando sobre $P_{N_{9}}$ e $P_{N_{1}}$ obtemos

$$
\int \mathcal{D} P_{N_{0}} e^{-\dot{y} \int d z \int d y P_{N_{0}}\left(N_{1}-1\right)} \int \mathcal{D} P_{N_{i}} e^{i \int d \tau \int d_{i z} P_{N_{1}} N_{0}}=\delta\left(N_{0}\right) \delta\left(N_{1}-1\right)
$$

Os argumentos dos deltas funcionais mostram que fixamos o gauge conforme. $S_{e f}$ é dada agora por

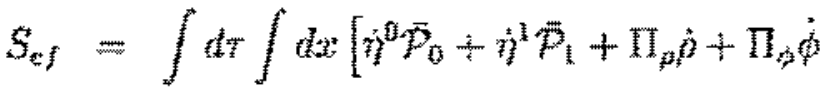

$$
\begin{aligned}
& -\tilde{\eta}^{0} \mathcal{P}_{1}-\tilde{\eta}^{1} \mathcal{P}_{0}-G_{0} N_{1}+\tilde{p}_{0} \eta^{1} N_{1} \\
& +2 \overline{\mathcal{P}}_{0} \eta^{l y} N_{1}+\overline{\mathcal{P}}_{1}^{\prime} \eta^{\mathrm{a}} N_{1}+2 \overline{\mathcal{P}}_{1} \eta^{0 /} N_{1}+\overline{\mathcal{P}}_{0} \mathcal{P}_{0} \\
& +G_{1} N_{0}-\overline{\mathcal{P}}_{0}^{\prime} \eta^{0} N_{0}-2 \overline{\mathcal{P}}_{0} \eta^{0 \prime} N_{0}-\tilde{\mathcal{P}}^{1 \prime} \eta^{1} N_{0} \\
& \left.-2 \bar{p}_{1} \eta^{1 t} N_{0}-\bar{p}_{1} \mathcal{P}_{1}\right]
\end{aligned}
$$

e $z$ por

$$
\begin{aligned}
& Z=\int \mathcal{D} \rho \mathcal{D} \Pi_{\rho} \mathcal{D} \phi \mathcal{D} \Pi_{\phi} \mathcal{D} N_{0} \mathcal{D} P_{N_{0}} \mathcal{D} N_{1} \mathcal{D} P_{N_{1}} \mathcal{D} \eta^{0} \mathcal{D} \overline{\mathcal{P}}_{0} \mathcal{D} \eta^{\mathrm{i}} \mathcal{D} \bar{p}_{1} \\
& \mathcal{D} \tilde{\eta}^{\mathrm{b}} \mathcal{D} \mathcal{P}_{0} \mathcal{D} \bar{\eta}^{-1} \mathcal{D} \mathcal{P}_{1} \delta\left(N_{0}\right) \delta\left(N_{1}-1\right) e^{i S_{e}}
\end{aligned}
$$

Integrando sobre $N_{0}$ e $N_{1}$ a ação efetiva toma-se

$$
\begin{aligned}
S_{e f} & =\int d \tau \int d x\left[\dot{\eta}^{0} \overline{\mathcal{P}}_{0}+\dot{\eta}^{1} \overline{\mathcal{P}}_{1}+\Pi_{\rho} \dot{\rho}+\Pi_{\phi} \dot{\phi}\right. \\
& -\tilde{\eta}^{0} \mathcal{P}_{1}-\bar{\eta}^{1} \mathcal{P}_{0}-G_{0}+\overline{\mathcal{P}}_{0} \eta^{2 /} \\
& \left.+\overline{\mathcal{P}}_{1} \eta^{o}+\overline{\mathcal{P}}_{0} \mathcal{P}_{0}-\overline{\mathcal{P}}_{1} \mathcal{P}_{1}\right]
\end{aligned}
$$

Integrando agora sobre $\bar{p}_{0}$ 


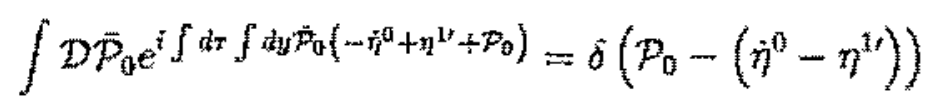

e integrando sobre $p_{0,}$ obtemos para a açảo efetiva

$$
\begin{aligned}
& S_{e f}=\int d r \int d y\left[\dot{\eta}^{2} \bar{p}_{1}+\Pi_{\rho} \dot{\rho}+\Pi_{\phi} \dot{\dot{\phi}}-\bar{\eta}^{-\hat{q}^{2} \mathcal{P}_{1}}\right. \\
& \left.-\bar{\eta}^{1}\left(\eta^{0}-\eta^{1}\right)-G_{0}+\bar{p}_{1} \eta^{0 *}-\bar{p}_{1} P_{1}\right]
\end{aligned}
$$

Integrando sobre $\bar{p}_{1}$

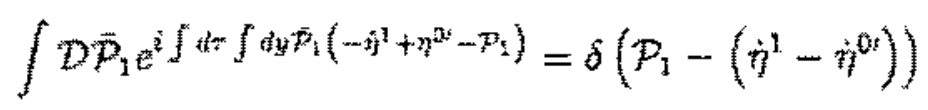

e integrando sobre $\mathcal{P}_{1}$ obtemos para $S_{e f}$

$$
S_{e f}=\int d \tau \int d y\left[\Pi_{\rho} \dot{\rho}+\Pi_{\dot{\psi}} \dot{\phi}-\bar{\eta}^{0}\left(\dot{\eta}^{1}-\eta^{0 t}\right)-\bar{\eta}^{1}\left(\dot{\eta}^{0}-\eta^{\mathrm{l}}\right)-G_{0}\right]
$$

Integrando sobre $\tilde{\eta}^{0} e \bar{\eta}^{1}$

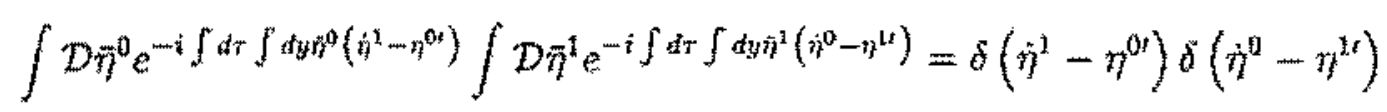

e a açào efetiva torna-so

$$
S_{e j}=\int d r \int d y\left(\Pi_{\rho} \dot{\rho}+\Pi_{\dot{\phi}} \dot{\phi}-G_{0}\right)
$$

Agora a funçäo de partição é dada por

$$
\begin{aligned}
Z= & \int D \eta^{0} D \eta^{1} D \rho D \Pi_{\phi} D_{\phi} D \Pi_{\phi} \delta\left(\dot{\eta}^{1}-\eta^{0}\right) \delta\left(\eta^{0}-\eta^{1}\right) \\
& e^{\left(\int d x \int d y\left(\Pi_{\phi} \dot{\partial}+\Pi_{\phi} \dot{\phi}-\sigma_{0}\right)\right)}
\end{aligned}
$$

A primeira funçăo delta no integrando da açāo acima pode ser escríta como 


$$
\delta\left(\eta^{1}-\eta^{0 r}\right)=\delta\left[\hat{\partial}_{0}\left(\eta^{1}-\frac{1}{\partial_{0}} \eta^{0 t}\right)\right]=\frac{1}{\operatorname{det}\left(\partial_{0}\right)} \delta\left(\eta^{1}-\frac{1}{\partial_{0}} \eta^{0 h}\right)
$$

Integrando sobre $\eta^{1}$

$$
\frac{1}{d e t\left(\partial_{0}\right)} \int D \eta^{t} \delta\left(\eta^{2}-\frac{1}{\partial_{0}} \eta^{0}\right) \delta\left(\eta^{0}-\eta^{u}\right)=\frac{1}{d e t\left(\partial_{0}\right)} \delta\left(\eta^{0}-\frac{1}{\partial_{0}} \eta^{0 \eta}\right)
$$

A função delta que aparece acima pode ser escrita como

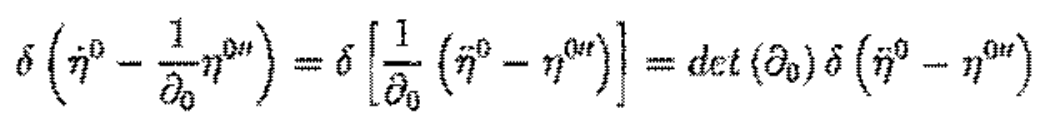

Assim 2 pode ser eserita como

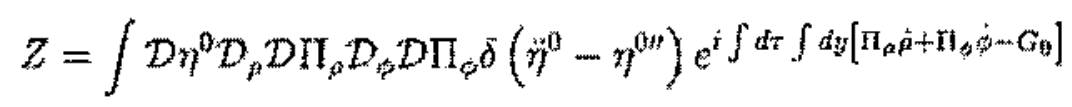

Concluimos, do resultado acima, que para o modelo de CGHS os fantasmas estão desacoplados dos campos da teoria podendo ser integrados na funçäo de partiçăo. Os fantasmas so contribuirăo para o termo central da ádgebra do tensor momento-energia, conforme será mostrado na seção 3.7 . 


\subsection{Forma Quadrática do Modelo de CGHS}

Os vinculos escritos na forma original (2.57) podem ser escritos na forma quadrática utilizando-se duas transformaçoes canónicas [15]. A primeira é obtida através do funcional gerador

$$
\begin{aligned}
& F\left(\rho_{1} \Pi_{\phi ;} \rho^{\mathrm{a}} \rho^{\mathrm{l}}\right)=-\int_{-\infty}^{\infty} d \sigma e^{\rho(\alpha)}\left[\rho^{0}(\sigma) \sinh \theta(\sigma)-\rho^{\mathrm{b}}(\sigma) \cosh \theta(\sigma)\right] \\
& \theta(\sigma)=\frac{1}{2} \int_{-\infty}^{\sigma} d \tilde{\sigma} \Pi_{b}(\tilde{\sigma})
\end{aligned}
$$

onde $\rho^{a}(a=0,1)$ são as novas varíkvis. Os momentos canônicamente conjugados a $\rho^{a}(\sigma)$ são as varitavests $p_{n}(\sigma)$. As novas variăveis obedecem juntamente com $\phi$ e $\Pi_{\rho}$ a

$$
\begin{aligned}
p_{a} & =-\frac{\partial F}{\partial p^{\alpha}} \\
\phi & =-\frac{\partial F}{\partial \Pi^{\prime}} \\
\Pi_{p} & =\frac{\partial F}{\partial \rho}
\end{aligned}
$$

Usando (3.46) em (3.47) podemos escrever os vínculos como

$$
\begin{aligned}
& G_{\mathfrak{O}}=-p^{a f} \epsilon_{a}^{b} p_{b}-\Lambda p_{a} p^{a} \\
& G_{1}=p_{a} p^{a t}{ }^{b}
\end{aligned}
$$

onde $\epsilon^{a b}=-\epsilon^{b a}$ e $\epsilon^{01}=1$ e os indices sẫo levantados abaixados com $\eta_{a b}=d i a g(-1,1)$. A segunda trantśormação canônica é dada por

$$
\begin{aligned}
\Pi_{a} & =-\Lambda p_{a}+\frac{1}{2} \epsilon_{a \hat{b}} \rho^{b} \\
r^{a} & =-\frac{\rho^{a}}{\Lambda},
\end{aligned}
$$


onde $\left(\tau_{a}, \Pi^{a}\right)$ constitui um par canônicamente conjugado. Esta filtima transformaçăo nos permite serever os vínculos na forma quadrática

$$
\begin{aligned}
& G_{0}=-\frac{1}{2}\left(\frac{2}{\Lambda} \Pi_{a} \Pi^{a}+\frac{\Lambda}{2} r_{a}^{\prime} r^{a \prime}\right) \\
& G_{1}=\Pi_{a} r^{a \prime}
\end{aligned}
$$

A transformaçäo canônica que resume as duas transformaçōes indicadas, de forma única é a seguinte [31]

$$
\begin{aligned}
& \Pi_{0}-\frac{\Lambda}{2} r^{1 t}=\Lambda e^{p} \sinh \theta \\
& \Pi_{1}+\frac{\Lambda}{2} t^{r}=-\Lambda e^{p} \cosh \theta \\
& A r^{\theta}=-e^{-\theta}\left(2 \phi^{2} \cosh \theta-\Pi_{p} \sinh \theta\right) \\
& \Lambda r^{\mathrm{i}}=e^{-\rho}\left(\Pi_{p} \cosh \theta-2 \phi^{\prime} \sinh \theta\right) \\
& \theta(\sigma)=\frac{1}{2} \int_{-\infty}^{\sigma} d \sigma \Pi_{\phi}(\sigma)
\end{aligned}
$$

Aplicando (3.5t) na equação (3.45) obtemos

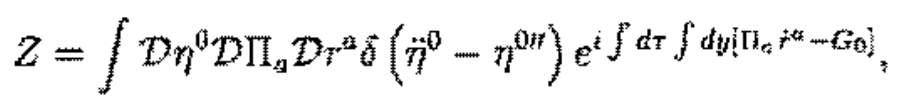

onde o vínculo $G_{0}$ é dado acima.

Observamos agora cue em $Z$ só aparecem integrais gaussianas nos campos.

Miković [14] utiliza outro método para obter a transformaçäo canônica que torna os vínculos quadráticos. O método é baseado na transformaçäo de Bäcklund, obtido a partir da teoria de Lisuville. 


\subsection{Quantização BRST-BFV do Modelo de CGHS na Forma Quadrática}

Para quantizarmos o modelo de CGHS com os vinculos escritos na forma quadrática seguimos o procedimento de quantizaçăo BRST-BFV idêntico ao utilizado na seção 3.2. Obtemos inicialmente a identificaryão dos vinculos com os respectivos pares de fantasmas

$$
\begin{array}{r}
C_{0} \rightarrow\left(\xi^{0}, \vec{\Sigma}_{0}\right) \\
G_{1} \rightarrow\left(\xi, \xi_{1}\right) \\
P_{B_{0}} \rightarrow\left(\xi^{1}, \Sigma_{1}\right) \\
P_{N_{1}} \rightarrow\left(\xi, \Sigma_{0}\right)
\end{array}
$$

onde $P_{N_{0}}$ e $P_{N_{1}}$ săo os momentos canônicamente conjugados aos multiplicadores de Lagrange $N_{0}$ e $N_{1}$. Os vínculos săo bosönicos como no caso da seça 3.2 e portanto os fantasmas possuem paridade 1. Estes fantasmas respeitam os seguintes PB's básicos

$$
\begin{aligned}
\left\{\xi^{i}, \xi^{j}\right\} & =0 \\
\left\{\Sigma_{i}, \Sigma_{j}\right\} & =0 \\
\left\{\bar{\xi}^{i}, \bar{\xi}^{j}\right\} & =0 \\
\left\{\bar{\Sigma}_{i}, \bar{\Sigma}_{j}\right\} & =0 \\
\left\{\bar{\xi}^{i}, \xi^{j}\right\} & =0 \\
\left\{\Sigma_{i}, \Sigma_{j}\right\} & =0 \\
\left\{\xi^{i}, \Sigma_{j}\right\} & =-\delta_{j}^{i} \delta(x-y) \\
\left\{\bar{\xi}_{j}, \Sigma_{j}\right\} & =-\delta^{i}{ }_{j} \delta(x-y)
\end{aligned}
$$

A álgebra dos vínculos ể a mesma obtida no caso anterior (seção 3.2 )

$$
\left\{G_{0}(x), G_{0}(y)\right\}=\delta^{\prime}(x-y)\left[G_{1}(x)+G_{1}(y)\right]
$$




$$
\begin{aligned}
& \left\{G_{1}(x), G_{0}(y)\right\}=\delta^{\prime}(x-y)\left[G_{0}(x)+G_{0}(y)\right] \\
& \left\{G_{1}(x), G_{1}(y)\right\}=\delta^{\prime}(x-y)\left[G_{1}(x)+G_{1}(y)\right]
\end{aligned}
$$

As constantes de estrutura sāo também as mesmas do caso nầo quadrático da seçăo $3.2 \mathrm{e}$ säo dadas por

$$
\begin{aligned}
& C_{00}^{0}=0 \\
& C_{00}^{1}=\delta^{t}(x-y) \delta(x-z)+f^{f}(x-y) \delta(y-z) \\
& C_{10}^{0}=\partial^{f}(x-y) \delta(x-z)+f^{f}(x-z) \delta(y-z) \\
& C_{10}^{1}=0 \\
& C_{12}^{0}=0 \\
& C_{11}^{1}=\delta^{f}(x-y) b(x-z)+f^{t}(x-y) \delta(y-z)
\end{aligned}
$$

A partir destes resultados, concluimos que a carga BRST possui a mesma forma da obtida na seğa 3.2

$$
\begin{aligned}
Q & =\int d x\left[\xi^{0} G_{0}+\xi^{1} G_{1}\right]+\int d x\left[\Sigma_{1} P_{N_{0}}+\Sigma_{0} P_{N_{2}}\right] \\
& +\int d x\left[\bar{\Sigma}_{0} \xi^{1} \xi^{0 \prime}+\bar{\Sigma}_{0} \xi^{0} \xi^{1 \prime}\right]+\int d x\left[\bar{\Sigma}_{1} \xi^{0} \xi^{0 \prime}+\Sigma_{1} \xi^{1} \xi^{1 \prime}\right]
\end{aligned}
$$

As variaçōes dos campos agora sâo dadas por

$$
\begin{aligned}
& \delta r^{\circ}=\frac{2}{A} \xi^{0} \Pi_{0}+\xi^{2} r^{0} \\
& \delta r^{2}=-\frac{2}{\Lambda} \xi^{0} \Pi_{1}+\xi^{1} r^{1} \\
& \delta \Pi_{0}=\frac{\Lambda}{2}\left(\xi^{0 t} p^{0 t}+\xi^{0} q^{0 i t}\right)+\xi^{1 r} \Pi_{0}+\xi^{\mathrm{l}} \Pi_{0}^{t}
\end{aligned}
$$

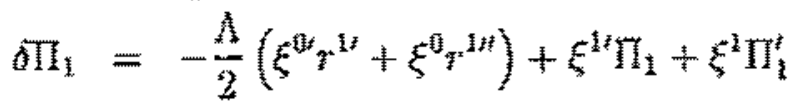

$$
\begin{aligned}
& \partial \xi^{0}=\xi^{0 t} \xi^{1}+\xi^{i t} \xi^{0}
\end{aligned}
$$




$$
\begin{aligned}
\delta \xi^{1} & =\xi^{0} \xi^{0}+\xi^{1 t} \xi^{1} \\
\delta \Sigma_{0} & =\delta \Sigma_{1}=0 \\
\delta \tilde{\Sigma}_{0} & =-G_{0}+\bar{\Sigma}_{0}^{\prime} \xi^{1}+2 \bar{\Sigma}_{0} \xi^{1 r}+\bar{\Sigma}_{1}^{\prime} \xi^{0}+2 \tilde{\Sigma}_{1} \xi^{0 \prime} \\
\delta \bar{\Sigma}_{1} & =-G_{1}+\bar{\Sigma}_{0}^{\prime} \xi^{0}+2 \bar{\Sigma}_{0} \xi^{0 t}+\bar{\Sigma}_{1}^{\prime} \xi^{1}+2 \bar{\Sigma}_{1} \xi^{1 r} \\
\delta P_{N_{0}} & =\delta P_{N_{1}}=0 \\
\delta N_{0} & =\Sigma_{1} \\
\delta N_{1} & =\Sigma_{0} \\
\delta \bar{\xi}^{0} & =-P_{N_{1}} \\
\delta \bar{\xi}^{1} & =-P_{N_{0}}
\end{aligned}
$$

O termo de fixação de gauge é dado por

$$
\Psi=\int d x\left[\bar{\xi}^{0} \chi_{0}+\bar{\xi} \chi_{1}-\bar{\Sigma}_{0} N_{1}+\bar{\Sigma}_{1} N_{0}\right]
$$

Assim obtemos para $\{Q, \Psi\}$

$$
\begin{aligned}
& \{Q, \Psi\}=\int d x\left[-P_{X_{1} \chi_{0}}+\vec{\xi}^{0} \partial_{0}-P_{S_{0}} \chi_{1}+\vec{\xi} \delta \chi_{1}\right. \\
& -\left(-G_{0}+\bar{\Sigma}_{0}^{\prime} \xi^{1}+2 \Sigma_{0} \xi^{1 \prime}+\bar{\Sigma}_{1}^{\prime} \xi^{0}\right. \\
& \left.+2 \bar{\Sigma}_{1} \xi^{E_{i}}\right) N_{1}-\bar{\Sigma}_{0} \Sigma_{0}+\left(-G_{1}+\Sigma_{0}^{\prime} \xi^{0}\right. \\
& \left.\left.+2 \bar{\Sigma}_{0} \xi^{0 t}+\bar{\Sigma}_{1}^{t} \xi^{1}+2 \bar{\Sigma}_{1} \xi^{1 !}\right) N_{0}+\Sigma_{1} \Sigma_{1}\right]
\end{aligned}
$$

A açăo efetiva é dada por

$$
\begin{aligned}
S_{e f} & =\int d r\left\{\int d x \left[P_{N_{0}} \dot{N}_{0}+P_{N_{1}} \dot{N}_{1}+\dot{\xi}^{0} \dot{\Sigma}_{0}+\dot{\xi}^{1} \dot{\Sigma}_{1}+\dot{\xi}_{\hat{q}} \Sigma_{0}\right.\right. \\
& \left.\left.+\dot{\xi} \Sigma_{1}+\Pi_{0} \dot{r}^{0}+\Pi_{1} \dot{q}^{1}-\mathcal{H}_{c}\right]-\{Q, \Psi\}\right\}
\end{aligned}
$$

ou 


$$
\begin{aligned}
S_{e f} & =\int d \tau \int d x\left\{P_{N_{0}} \dot{N}_{0}+P_{N_{1}} \dot{N}_{1}+\dot{\xi}^{0} \tilde{\Sigma}_{0}+\dot{\xi}^{1} \bar{\Sigma}_{1}\right. \\
& +\dot{\bar{\xi}}^{0} \Sigma_{0}+\dot{\bar{\xi}}_{1} \Sigma_{1}+\Pi_{0} \dot{r}^{0}+\Pi_{1} \dot{r}^{1} \\
& +P_{N_{1}} \chi_{0}-\bar{\xi}^{0} \delta \chi_{0}+P_{N_{0}} \chi_{1}-\bar{\xi}^{1} \delta \chi_{1} \\
& +\left(-G_{0}+\bar{\Sigma}_{0}^{\prime} \xi^{1}+2 \bar{\Sigma}_{0} \xi^{1 \prime}+\bar{\Sigma}_{1}^{\prime} \xi^{0}\right. \\
& \left.+2 \bar{\Sigma}_{1} \xi^{0 \prime}\right) N_{1}+\bar{\Sigma}_{0} \Sigma_{0}+\left(G_{1}-\bar{\Sigma}_{0}^{\prime} \xi^{0}\right. \\
& \left.\left.-2 \bar{\Sigma}_{0} \xi^{0 \prime}-\bar{\Sigma}_{1}^{\prime} \xi^{1}-2 \bar{\Sigma}_{1} \xi^{1 \prime}\right) N_{0}-\bar{\Sigma}_{1} \Sigma_{1}\right\}
\end{aligned}
$$

Para fixarmos o gauge conforme devemos ter para as funções de fixação de gauge

$$
\begin{aligned}
& \chi_{0}=\frac{N_{0}}{\epsilon} \\
& \chi_{3}=\frac{N_{1}-1}{\epsilon}
\end{aligned}
$$

e assim obtemos com auxílio de (3.58)

$$
\begin{aligned}
& \delta \chi_{0}=\frac{\Sigma_{1}}{\epsilon} \\
& \delta \chi_{1}=\frac{\Sigma_{0}}{\epsilon}
\end{aligned}
$$

Usamos agora a seguinte transformação

$$
\begin{aligned}
P_{N_{0}} & =\epsilon P_{N_{0}} \\
P_{N_{1}} & =\epsilon P_{N_{1}} \\
\bar{\xi}^{0} & =\epsilon \bar{\xi}^{0} \\
\bar{\xi}^{1} & =\epsilon \bar{\xi}^{1}
\end{aligned}
$$

Usando (3.64) e (3.65) em (3.62) 


$$
\begin{aligned}
& S_{e j}=\int d \tau \int d x\left\{c P_{N_{9}} \dot{N}_{0}+\epsilon P_{N_{2}} \dot{N}_{1}+\xi^{0} \tilde{\Sigma}_{0}+\xi^{1} \Sigma_{1}\right.
\end{aligned}
$$

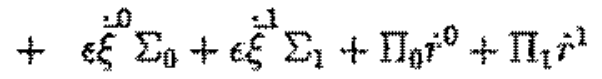

$$
\begin{aligned}
& +P_{N_{1}} N_{0}-\xi_{5} \Sigma_{1}+P_{N_{0}}\left(N_{1}-1\right)-Z_{0} \Sigma_{0} \\
& +\left(-G_{0}+\Sigma_{0}^{\prime} \xi^{1}+2 \Sigma_{0} \xi^{1 t}+\Sigma_{1}^{z} \xi^{0}\right. \\
& \left.+2 \bar{\Sigma}_{1} \xi^{0 \prime}\right) N_{1}+\bar{\Sigma}_{10} \Sigma_{0}+\left(G_{1}-\bar{\Sigma}_{0}^{\prime} \xi^{0}\right. \\
& \left.\left.-2 \tilde{\Sigma}_{0} \xi^{0 t}-\tilde{\Sigma}_{1}^{t} \xi^{1}-2 \Sigma_{1} \xi^{1 t}\right) N_{0}-\Sigma_{1} \Sigma_{1}\right\}
\end{aligned}
$$

e tomando o limite $\epsilon \rightarrow 0$

$$
\begin{aligned}
S_{e f} & =\int d r \int d x\left\{\dot{\xi}^{0} \Sigma_{0}+\xi^{1} \Sigma_{1}+\Pi_{0} \dot{r}^{0}+\Pi_{1} \dot{r}^{1}-\bar{\xi}^{0} \Sigma_{1}-\xi^{1} \Sigma_{0}\right. \\
& +P_{N_{2}} N_{0}+P_{N_{0}}\left(N_{1}-1\right)+\left(-G_{0}+\Sigma_{0}^{\prime} \xi^{1}+2 \Sigma_{0} \xi^{1 \prime}+\Sigma_{1}^{\prime} \xi^{0}+2 \bar{\Sigma}_{1} \xi^{0 \prime}\right) N_{1} \\
& \left.+\left(G_{1}-\bar{\Sigma}_{0}^{\prime} \xi^{0}-2 \bar{\Sigma}_{0} \xi^{0 \prime}-\bar{\Sigma}_{1}^{\prime} \xi^{1}-2 \Sigma_{1} \xi^{\prime \prime}\right) N_{0}+\Sigma_{0} \Sigma_{0}-\Sigma_{1} \Sigma_{1}\right\}
\end{aligned}
$$

De forma semelhante ao realizado na seçào 3.3 integramos a ação em $P_{N_{0}}$ e $P_{N_{1}}$, e assim fixamos o gauge conforme. Obtemos

$$
\begin{aligned}
S_{e f} & =\int d \tau \int d x\left\{\xi^{0} \bar{\Sigma}_{0}+\xi^{1} \bar{\Sigma}_{1}+\Pi_{0} \dot{r}^{0}+\Pi_{1} \dot{r}^{1}-\xi^{0} \Sigma_{1}-\xi^{1} \Sigma_{0}\right. \\
& \left.+\left(-G_{0}+\Sigma_{0} \xi^{1}+2 \Sigma_{0} \xi^{17}+\Sigma_{1} \xi^{0}+2 \Sigma_{1} \xi^{5}\right)+\Sigma_{0} \Sigma_{0}-\Sigma_{1} \Sigma_{i}\right\}
\end{aligned}
$$

Das equações fornecidas para as variaçöes $(3.58)$ verificamos que $\bar{\xi}^{\natural}$ e $\bar{\xi}^{1}$ năo são variáveis independentes. Vamos entäo integrar a açăo nestas duas variáveis.

Integrando a funça de partição en $\bar{\xi}^{0}$ e $\bar{\xi}$ obtemos deltas funcionais que fixam $\Sigma_{0}$ e $\Sigma_{1} \mathrm{em}$

$$
\sum_{n \rightarrow 1}=0
$$

Logo 


$$
\begin{aligned}
S_{e f} & =\int d \tau \int d x\left\{\dot{\xi}^{0} \bar{\Sigma}_{0}+\dot{\xi}^{1} \bar{\Sigma}_{1}+\Pi_{0} \dot{r}^{0}+\Pi_{1} \dot{r}^{1}\right. \\
& \left.+\left(-G_{0}+\bar{\Sigma}_{0}^{\prime} \xi^{1}+2 \bar{\Sigma}_{0} \xi^{1 \prime}+\bar{\Sigma}_{1}^{\prime} \xi^{0}+2 \bar{\Sigma}_{1} \xi^{0 \prime}\right)\right\}
\end{aligned}
$$

Realizando integraçōes por partes no sexto e oitavo termos, obtemos

$$
\begin{aligned}
S_{e f} & =\int d \tau \int d x\left\{\dot{\xi}^{0} \bar{\Sigma}_{0}+\dot{\xi}^{1} \bar{\Sigma}_{1}+\Pi_{0} \dot{r}^{0}+\Pi_{1} \dot{r}^{1}\right. \\
& \left.+\left(-G_{0}+\bar{\Sigma}_{0} \xi^{1 \prime}+\bar{\Sigma}_{1} \xi^{0 \prime}\right)\right\}
\end{aligned}
$$

ou

$$
\begin{aligned}
S_{e f} & =\int d \tau \int d x\left\{\Pi_{0} \dot{r}^{0}+\Pi_{1} \dot{r}^{1}-G_{0}\right. \\
& \left.+\bar{\Sigma}_{0}\left(\xi^{1 \prime}-\dot{\xi}^{0}\right)+\bar{\Sigma}_{1}\left(\xi^{0 \prime}-\dot{\xi}^{1}\right)\right\}
\end{aligned}
$$

usando a equação para o vínculo $G_{0}(3.50)$ na expressão acima obtemos

$$
\begin{aligned}
S_{e f} & =\int d \tau \int d x\left\{\frac{\Lambda}{4}\left(\dot{r}^{02}-r^{0 \prime 2}\right)-\frac{\Lambda}{4}\left(\dot{r}^{12}-r^{1 \prime 2}\right)\right. \\
& \left.+\bar{\Sigma}_{0}\left(\xi^{1 \prime}-\dot{\xi}^{0}\right)+\bar{\Sigma}_{1}\left(\xi^{0 \prime}-\dot{\xi}^{1}\right)\right\}
\end{aligned}
$$

Integrar a função de partição em $\bar{\xi}^{0}$ e $\bar{\xi}^{1}$, como fizemos acima, corresponde a escrever a ação on-shell, ou seja, a usar as equaçōes de movimento para $\Sigma_{0}$ e $\Sigma_{1}$ (3.69).

Notamos da equação acima que os campos estão desacoplados dos fantasmas. Percebemos ainda que possuimos um termo escalar com energia cinética positiva $r^{0}$ e outro com energia cinética negativa $r^{1}$. 


\subsection{Análise de Anomalias para o Modelo de CGHS}

\subsubsection{Cálculo da Anomalia}

Anomalias ocorrem quando simetrias de um sistema clássico năo podem ser mantidas ñ tooria quuântica.

Quando cuantizamos os campos do modelo de CGHS esperamos que álgebra dos vínculos expressa por $(3.55)$ seja preservada, uma vez que devemos seguir a prescrição de Dirac para transformar PB's em comutadores, ou seja

$$
\{A, B\} \rightarrow \frac{\mid A, B]}{\frac{t}{*}}
$$

Fututanto analisando a kyebra dos víntulos expressos na foma do tersor momentoenergia, verificamos a presenga de uma anomalia, conforme desmonstraremos a seguir.

Obtivemos os vínculos na forma quadrática en (3.50) escritos em funçäo dos campos escalares $r^{0}$ e $r^{\mathrm{I}}$. Para obter a contribuição dos fantasmas nos vinculos vamos reescrevê-los na forma [32.

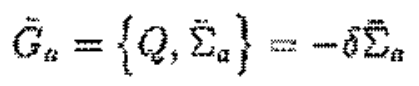

Usando $(3.58)$ obtemos

$$
\begin{aligned}
& \tilde{G}_{0}=G_{0}-\left(\bar{\Sigma}_{0}^{\prime} \xi^{1}+2 \Sigma_{0} \xi^{t \prime}+\Sigma_{1} \xi^{0}+2 \bar{\Sigma}_{0} \xi^{0 t}\right)
\end{aligned}
$$

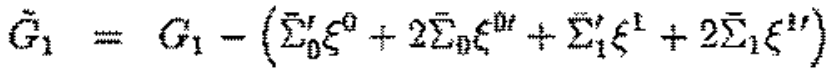

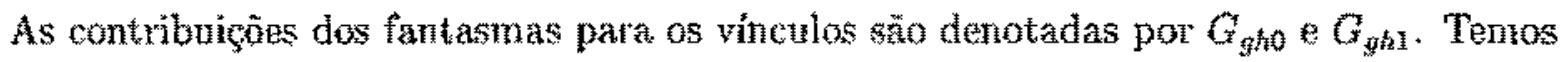

$$
\begin{aligned}
& G_{g h 0}=-\left(\bar{\Sigma}_{0}^{\prime} \xi^{l}+2 \bar{\Sigma}_{0} \xi^{1 \prime}+\bar{\Sigma}_{x}^{\prime} \xi^{0}+2 \bar{\Sigma}_{1} \xi^{0 r}\right) \\
& G_{z h 1}=-\left(\Sigma_{0} \xi^{0}+2 \bar{\Sigma}_{0} \xi^{\mathrm{n} t}+\bar{\Sigma}_{1} \xi^{1}+2 \bar{\Sigma}_{1} \xi^{i t}\right)
\end{aligned}
$$

Vamos agora reescrever $G_{0}$ e $Q_{1}$ de forma aclitur análise da anomalia. Utilizando as equacoes para os momentos canônicamente conjugados aos campos $r^{\circ}$ e $r^{2}$ (veja a aça expressa em $(3.73)$ ) 


$$
\Pi_{a}=-\frac{\Lambda}{2} \dot{r}_{a}
$$

Podemos escrever para os vínculos (3.50)

$$
\begin{aligned}
& G_{0}=-\frac{A}{4}\left[i_{n} r^{a}+r^{a t} r_{n}^{t}\right] \\
& G_{1}=-\frac{A}{2}\left[i_{n} z^{a t}\right]
\end{aligned}
$$

Usandó zoordenadas do cone de luz

$$
\begin{aligned}
& x^{+}=\frac{x^{0}+x^{1}}{2} \\
& x^{-}=\frac{x^{0}-x^{4}}{2}
\end{aligned}
$$

podemos escrever para $G_{0}$ e $G_{1}$

$$
\begin{aligned}
& G_{0}=-\frac{\Lambda}{8}\left[\partial_{4} r_{a} \partial_{4} r^{a}+\partial_{-} r_{i t} \partial_{m} r^{n}\right] \\
& G_{1}=-\frac{\Lambda}{8}\left[\partial_{+} r_{a} \partial_{4} r^{a}-\partial_{-} r_{t} \partial_{m} r^{a}\right]
\end{aligned}
$$

Reescrevemos $G_{p f o}$ e $G_{\text {sh }}$ utilizando a transformação

$$
\begin{aligned}
& \xi^{0}=\frac{1}{2}(c+d) \\
& \xi^{1}=\frac{1}{2}(c-d) \\
& \bar{\Sigma}_{0}=\frac{1}{2}(\bar{c}+\vec{d}) \\
& \bar{\Sigma}_{1}=\frac{1}{2}(\bar{c}-\vec{d})
\end{aligned}
$$

obtemos 


$$
\begin{aligned}
& G_{g h v}=-\frac{1}{2}\left[\vec{c} c+2 \bar{c} c^{\prime}-\bar{d} d-2 \vec{d} d^{\prime}\right] \\
& G_{g h 1}=-\frac{1}{2}\left[\vec{c} c+2 \bar{c} c^{\prime}+\vec{d} d+2 \bar{d} d^{\prime}\right]
\end{aligned}
$$

ou em coordenadas do cone de luz

$$
\begin{aligned}
& C_{a k 0}=-\frac{1}{4}\left[\left(\partial_{+} \vec{c}-\partial_{-} \vec{c}\right) c+3 \vec{c}\left(\partial_{+} c-\partial_{-} d-\left(\partial_{+} \bar{d}-\partial_{-} \vec{d}\right) d-2 \bar{d}\left(\partial_{+} d-\partial_{-} d\right]\right.\right. \\
& G_{g h 1}=-\frac{1}{4}\left[\left(\partial_{+} \bar{c}-\partial_{-} \bar{c}\right) c+2 \bar{c}\left(\partial_{+} c-\partial_{-} c\right)+\left(\partial_{+} \bar{l}-\partial_{-} d\right) d+2 \bar{d}\left(\partial_{+} d-\partial_{-} d\right)\right]
\end{aligned}
$$

Agora definimos

$$
\begin{aligned}
& G_{0++}=\frac{\left(\tilde{G}_{0}+\tilde{G}_{1}\right)}{2} \\
& G_{0-}=\frac{\left(\tilde{G}_{0}-\tilde{G}_{1}\right)}{2}
\end{aligned}
$$

a usando $(3.76)(3.81)(3.83)$ e (3.84) na squaça keima obtemos

$$
\begin{aligned}
& G_{0+}=-\frac{\Lambda}{8}\left(\partial_{+} \psi_{a} \partial_{+} \gamma^{n}\right)-\frac{1}{4}\left[\left(\partial_{+} \bar{c}-\partial_{-} \bar{c}\right) c+2 \bar{c}\left(\partial_{+} c-\partial_{-} c\right)\right] \\
& G_{0-}=-\frac{A}{8}\left(\partial_{-} \tau_{0} \partial_{-} z^{a}\right)+\frac{1}{4}\left[\left(\partial_{+} d-\partial_{-} d\right) d+2 d\left(\partial_{4} d-\partial_{-} d\right)\right]
\end{aligned}
$$

Agora vamos nos referir a equaçăo (3.73). A contribuiçăo dos fantasmas para a lagrangeana efetiva é dada por

$$
L_{g \hat{h}}=\bar{\Sigma}_{0}\left(\xi^{\prime \prime}-\dot{\xi}^{0}\right)+\Sigma_{1}\left(\xi^{0 r}-\dot{\xi}^{\prime}\right)
$$

Usando a transformação $(3.82)$ obtemos 


$$
L_{3 h}=-\frac{1}{2} c \partial_{-} c-\frac{1}{2} \partial_{4} d
$$

desta açăo derivamos as equaçöes de movimento

$$
\partial_{-} c=\partial_{-} c=\partial_{4} d=\partial_{4} z=0
$$

Utilizando estas equaçöes em $G_{0+}$ e $C_{0-}$ chegemos ao resultado

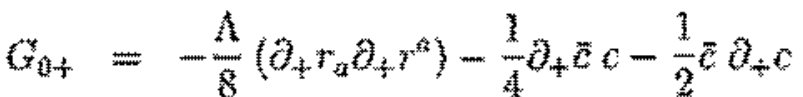

$$
\begin{aligned}
& G_{a-1}=-\frac{A}{8}\left(\partial_{-} r_{a} \partial_{-} t^{a}\right) \cdots \frac{1}{4} \partial_{-} d d-\frac{1}{2} d \partial_{-} d
\end{aligned}
$$

Verificaremos agora, que os vínculos escritos na forma de $G_{0+}$ e $G_{0-}$ representam tespectivamente as componentes do tensor momento energia $T_{+\rightarrow}$ e $T_{--}$. De fato, dada una lagrangeana para um campo escalar $\varphi$ com energia cinética positiva

$$
L=-\frac{1}{2} \int d x \partial^{\mu} \varphi \partial_{\mu} \varphi
$$

e usando a definiçăo usual para o tensor momento energia

$$
T_{\mu \mu}=\frac{1}{\sqrt{-g}} \frac{\partial L}{\partial g^{\mu \nu}}
$$

obtemos

$$
T_{p, \nu}=-\frac{1}{2} \partial_{\mu} \varphi \partial_{u} \varphi-\frac{1}{2} \eta_{\mu \nu} L
$$

que nas coordenadas do cone de luz toma a forma

$$
\begin{aligned}
& T_{ \pm \pm}=-\frac{1}{2} \partial_{ \pm} \varphi \partial_{ \pm} \psi \\
& T_{+--}=0
\end{aligned}
$$




$$
T_{p \nu}=\frac{1}{\sqrt{-g}} \frac{\partial L}{\partial g^{H}}
$$

Comparando (3.94) com (3.90) verificamos que elas representam a mesma equaçăo (a menos de uma constante). Para o caso de um campo escalar con energia cinética negatiła basta inverter o sinal de $(3.95),(3.93)$ e $(3.94)$. Fimportante notar que $G_{0+}$ e $G_{0-}$ contém a contribuiçăo de um temo com energia cinética positiva referente a $\tau^{0}$ e outro com energia cinética negativa referente a $r^{1}$.

Para os fantasmas calculamos também o tensor momento energia partindo da seguinie lagrangeana

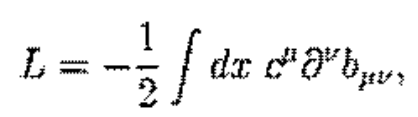

onde $b_{p \nu}$ é um tensor de segunda ordem simếtrico e de traço nulo ou seja

$$
b_{j k}^{\mu}=0
$$

O tensor momento-energia neste caso, ế dado por (veja apêndice B)

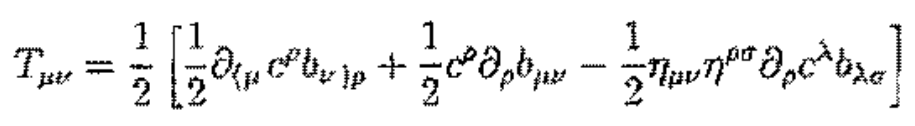

Nas coordenadas do cone de luz

$$
\begin{aligned}
& T_{ \pm \pm}=\frac{1}{2} \partial_{ \pm} c^{ \pm} b_{ \pm \pm}+\frac{1}{4} c^{ \pm} \partial_{ \pm} b_{ \pm \pm} \\
& T_{ \pm-m}=0
\end{aligned}
$$

Novamente, comparando (3.99) com o termo de (3.90) referente aos fantasmas, verificamos que estak expressões representam a mesma equaçäo.

$\mathrm{Da}$ álgebra (3.55) esperamos obter, após converter PB's em conutadores ou anticomutadores, tanto para os campos escalares quanto para os fantasmas (veìa apêndice $\mathrm{C}$ ) 


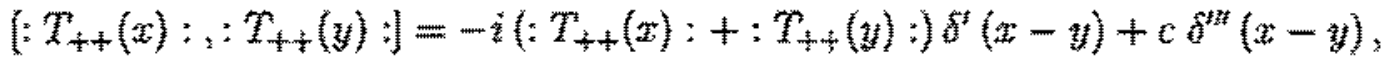

onde " $c$ " corresponde a termo central a sèr determinado.

Vamos considera novanonte o campo escalar em (3.91) e seu respectivo tensor $T_{p}$ (3.94). Utilizando expansōes em operadores de criação e aniquilaçào para os campos (veja apêndice C) obtemos

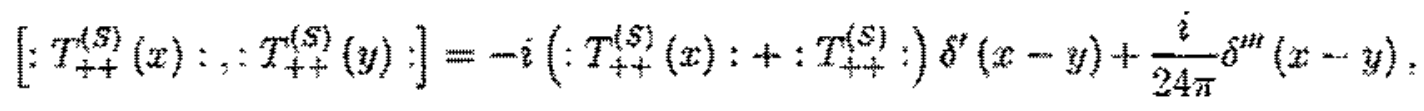

onde $T_{+}^{(S)}$ corresponde ao tensor momento-anengia para o campo escalar com energia cinética positiva. Para o campo escalar con energia cinética negativa verifcamos que o temo central năo muda de sinal (apêndice $C$ ).

Para a contritubuigato dos fantasmas obtemos (veja apandice D)

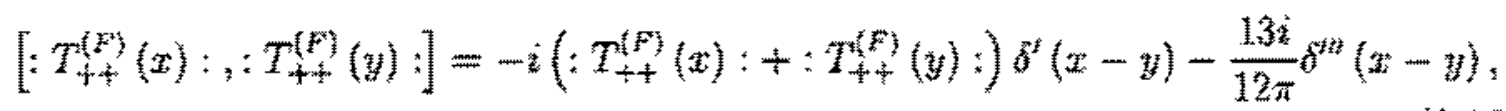

onde : $T_{++}^{(F)}$ : corresponde ao tensor momento-energia para os fantasmas. Os mesmos

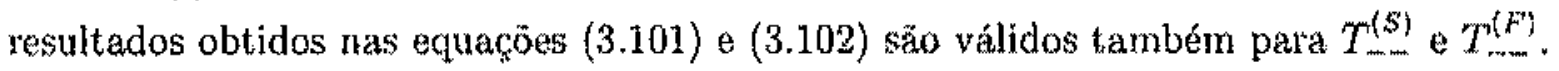

A hamiltoniana para o campo escalar obtida a partir de (3.91) pode ser escrita como

$$
n^{(s)}=\frac{1}{2} \int d a^{2}\left(m^{2}+p^{i 2}\right)
$$

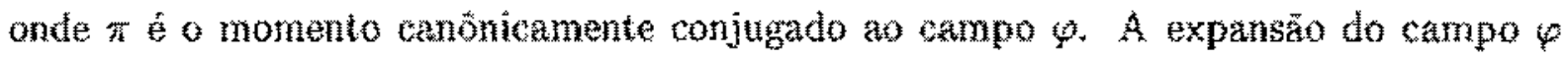
em operadores de criaçäo aniquilação fornece uma hamiltoniana cujos autovalores säo negativos (veja apêndice C).

A hamiltoniana para os fantasmas é obtida a partír de (3.96)

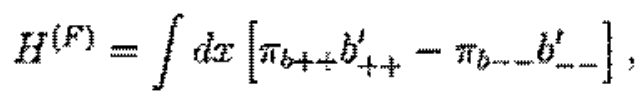


onde $\pi_{b++}$ e $\pi_{b--}$ sầo os momentos canônicanente conjugados aus campos $b_{++}$e $b_{-\ldots}$. Neste caso também os autovalores da hamiltoniana são negativos (vèja apêndice D).

É importante neste ponto discutîr o problema da definição do văcuo usada nos cálculos apresentados. Nos apêndices C e D usamos as seguintes expansōes para os campos

$$
\begin{aligned}
\varphi(x) & =\int_{-\infty}^{+\infty} \frac{d \mathbf{k}}{\sqrt{2 \pi}} \frac{1}{2|\mathbf{k}|}\left[a(\mathbf{k}) e^{i k x}+a^{\dagger}(\mathbf{k}) e^{-i k x}\right] \\
b_{++}(x) & =\sqrt{\frac{2}{\pi}} \int_{0}^{+\infty} d \mathbf{k}\left[b(\mathbf{k}) e^{i k x}+b^{\dagger}(\mathbf{k}) e^{-i k x}\right] \\
c^{+}(x) & =\frac{1}{\sqrt{\pi}} \int_{0}^{+\infty} d \mathbf{k}\left[c(\mathbf{k}) e^{i k x}+c^{\dagger}(\mathbf{k}) e^{-i k x}\right]
\end{aligned}
$$

Nos cálculos do termo central utilizamos tanto as definiçōes usuảs para o vácuo

$$
a|0>=b| 0>=c \mid 0>=0,
$$

onde $a, b$ e $c$ săo operadores de aniquilação, cono as definições invertidas onde os operadores de criaçăo passam a ser de aniquilaçăo

$$
a^{\dagger}\left|0>=b^{\dagger}\right| 0>=c^{4} \mid 0>=0
$$

Verificamos, que usando esta última convençào, a partir dos cálculos dos apêndices $\mathrm{C}$ e D, que o termo central tem o sinal invertido, tanto para o campo escalar quanto para os fantasmas. Resuminos nas tabelas abaixo os resultados obtidos incluindo os sinais das normas em cada caso para os campos escalares (analisados no apèndice C) 
Campo Escalat:

\begin{tabular}{|c|c|c|c|c|}
\hline $\begin{array}{c}\text { Sinal } \\
\text { Energia } \\
\text { Cinética }\end{array}$ & $\begin{array}{c}\text { Definiçào } \\
\text { Vácuo }\end{array}$ & $\begin{array}{c}\text { Sinal } \\
\text { da } \\
\text { Norma }\end{array}$ & $\begin{array}{c}\text { Autovalores da } \\
\text { Haniltoniana }\end{array}$ & $\begin{array}{c}\text { Termo Central } \\
\text { em Unidades de } \\
(-i / 24 \pi)\end{array}$ \\
\hline+ & $a \mid 0>=0$ & + & $E \geq 0$ & 1 \\
\hline+ & $a 10>=0$ & - & $E \leq 0$ & -1 \\
\hline- & $a \mid 0>=0$ & - & $E \geq 0$ & 1 \\
\hline- & $a^{\dagger} \mid 0>=0$ & + & $E \leq 0$ & -1 \\
\hline
\end{tabular}

Eantasmas

\begin{tabular}{|c|c|c|c|}
\hline $\begin{array}{c}\text { Sinal } \\
\text { Energia } \\
\text { Cinética }\end{array}$ & $\begin{array}{c}\text { Definição } \\
\text { Vácuo }\end{array}$ & $\begin{array}{c}\text { Autovalores da } \\
\text { Hamiltoniana }\end{array}$ & $\begin{array}{c}\text { Temo Central } \\
\text { em Unidades de } \\
(-1 / 24 \pi)\end{array}$ \\
\hline+ & $b|0>=c| 0>=0$ & $E \leq 0$ & 26 \\
\hline+ & $b^{\dagger}\left|0>=c^{\dagger}\right| 0>=0$ & $E \geq 0$ & -26 \\
\hline- & $b|0>=c| 0>=0$ & $E \leq 0$ & 26 \\
\hline- & $b^{\dagger}\left|0>=c^{\dagger}\right| 0>=0$ & $E \geq 0$ & -26 \\
\hline
\end{tabular}

Os fantasmas possuem norma zero, e portanto näo possuem significado físico.

Os valores obtidos para " $\mathrm{c}$ " mostram nầ ser possivel climinar a anomalia combinando diferentes definições do vácuo para campos diferentes. Ao introduzimos um termo de superfície na açäo denominado termo de improvement, conseguimos eliminar a anomalia, conforme veremos na próxima seçâo. 


\subsubsection{Termo de Improvement para o Cancelamento da Anomalia}

Para anular a anomalia, vamos acrescentar um termo à ação (3.73), que näo altera a dinâmica do sistema. $O$ termo acrescido a ação é representado por $S_{I}$ e é conhecido como termo de improvement. $S_{F}$ é dado por

$$
S_{l}=\frac{1}{2} \sqrt{\frac{\Lambda}{2}} Q_{\mathrm{a}} \int d^{2} x \partial^{\mu} \partial_{y} r^{a}
$$

onde os $Q^{*}$ 's săo constantes abitrérias. Para constatarmos que $S_{l}$ năo altera o conteudo físico da teoria, basta aplicar o teorema de Gauss a (3.108) para verificar que $S_{1}$ é igual a zero (assumindo que $r^{a} \rightarrow 0$ no infinito).

Usando (3.95) obtemos para o tensor momento-energia

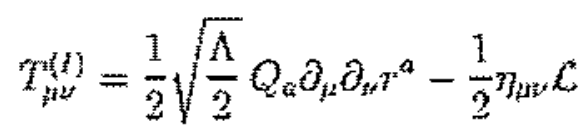

ou em coordenadas do cone de luz

$$
\begin{aligned}
& T_{ \pm \pm}^{(l)}=\frac{1}{2} \sqrt{\frac{\Lambda}{2}} Q_{n} \partial_{ \pm}^{2} r^{a} \\
& T_{+-}^{(l)}=0
\end{aligned}
$$

A nova lagrangeana $\left(L_{T}\right)$ é obtida a partir de $(3.73)$ e $(3.108)$

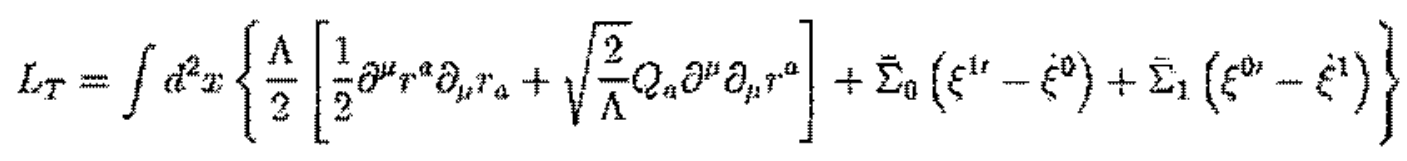

A partir de $L_{T}$ obtemos os novos vínculos

$$
\begin{aligned}
& \bar{G}_{0}=G_{0}+\sqrt{\frac{\hbar}{2}} Q_{n} 3^{n t} \\
& \bar{G}_{1}=G_{1}-\sqrt{\frac{2}{h}} Q_{a} \Pi^{a r},
\end{aligned}
$$


onde $G_{0}$ e $G_{1}$ são dados por (3.50).

Para a álgebra dos novos vínculos obtemos

$$
\begin{aligned}
& \left\{G_{0}(x), G_{0}(y)\right\}=\left[G_{1}(x)+G_{1}(y)\right] \delta^{\prime}(x-y) \\
& \left\{G_{1}(x), G_{0}(y)\right\}=\left[G_{0}(x)+G_{0}(y)\right] \delta^{\prime}(x-y)-Q_{a} Q^{a} \delta^{\prime \prime \prime}(x-y) \\
& \left\{G_{1}(x), G_{1}(y)\right\}=\left[G_{1}(x)+G_{1}(y)\right] \delta^{\prime}(x-y)
\end{aligned}
$$

Portanto teremos uma álgebra de vínculos de primeira classe se

$$
Q_{a} Q^{a}=0
$$

Este resultado esta de acordo com o obtido por Miković [14] [15].

Com esta condiçāo as constantes de estrutura são as mesmas obtidas para $G_{0}$ e $G_{1}$ (equações (3.56)). A nova carga de BRST pode ser obtida usando a seguinte substuição em $\{3.57)$

$$
\begin{aligned}
& G_{0} \longrightarrow \bar{G}_{0} \\
& G_{1} \longrightarrow \bar{G}_{1}
\end{aligned}
$$

obtemos

$$
\begin{aligned}
Q & =\int d x\left[\xi^{0} \bar{G}_{0}+\xi^{1} \bar{G}_{1}\right]+\int d x\left[\Sigma_{1} P_{N_{0}}+\Sigma_{0} P_{N_{1}}\right] \\
& +\int d x\left[\bar{\Sigma}_{0} \xi^{1} \xi^{0 \prime}+\bar{\Sigma}_{0} \xi^{0} \xi^{1 \prime}\right]+\int d x\left[\bar{\Sigma}_{1} \xi^{0} \xi^{0 \prime}+\bar{\Sigma}_{1} \xi^{1} \xi^{1 \prime}\right]
\end{aligned}
$$

As novas transformaçōes geradas por $Q$ sāo dadas abaixo

$$
\begin{aligned}
& \delta r^{0}=\frac{2}{\Lambda} \xi^{0} \Pi_{0}+\xi^{1} r^{0 \prime}+\sqrt{\frac{2}{\Lambda}} \xi^{1 \prime} Q^{0} \\
& \delta r^{1}=-\frac{2}{\Lambda} \xi^{0} \Pi_{1}+\xi^{1} r^{1 \prime}+\sqrt{\frac{2}{\Lambda}} \xi^{1 \prime} Q^{1}
\end{aligned}
$$




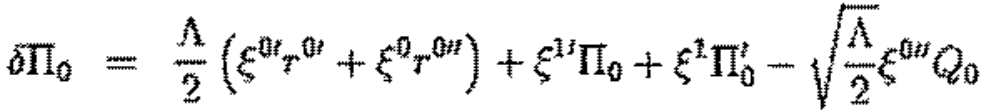

$$
\begin{aligned}
& \delta \Pi_{1}=-\frac{\Lambda}{2}\left(\xi^{0 t} r^{11}+\xi^{0} r^{1 n}\right)+\xi^{1 t} \Pi_{1}+\xi^{1} \Pi_{1}^{s}-\sqrt{\frac{\Lambda}{2}} \xi^{0 \prime \prime} Q_{1} \\
& \delta \bar{\Sigma}_{0}=-\bar{G}_{0}+\Sigma_{0}^{\prime} \xi^{1}+2 \bar{\Sigma}_{0} \xi^{1 t}+\bar{\Sigma}_{i}^{\prime} \xi^{0}+2 \bar{\Sigma}_{1} \xi^{0 \prime} \\
& \delta \bar{\Sigma}_{1}=-\bar{G}_{1}+\bar{\Sigma}_{0}^{\prime} \xi^{0}+2 \bar{\Sigma}_{0} \xi^{\prime \prime}+\bar{\Sigma}_{1}^{\prime} \xi^{1}+2 \bar{\Sigma}_{1} \xi^{1 \prime} \\
& \partial \xi^{0}=\xi^{0} \xi^{1}+\xi^{1 /} \xi^{0} \\
& \delta \xi^{1}=\xi^{1 H} \xi^{0}+\xi^{11} \xi^{1} \\
& \delta \Sigma_{0}=\delta \Sigma_{1}=0 \\
& \delta P_{N_{0}}=\delta P_{N_{1}}=0 \\
& \delta N_{0}=\Sigma_{1} \\
& \delta N_{1}=\Sigma_{0} \\
& \delta \xi^{0}=-P_{N_{1}} \\
& \delta \xi^{1}=-P_{N_{0}}
\end{aligned}
$$

Prosseguimos agora com o cáleulo do termo central para o tensor momento-energia levando-se em consideração o termo de improvement. Denotaremos por $T^{(I)}$ o tensor de improvement para o canpo escalar com termo cinético positivo (equaçăo (E.1)). O tensor momento-energia total neste caso,

$$
: T_{++}^{(T)}:=: T_{++}^{(S)}:+: T_{+}^{(t)}:
$$

e deve satisfazer a álgebra (3.100). Este cálculo é realizado no apêndice E. Obtemos

$$
\begin{aligned}
{\left[: T_{++}^{(T)}(x):, T_{++}^{(T)}(y):\right] } & =-i\left(: T_{++}^{(T)}(x):+: T_{++}^{T}(y):\right)^{\prime}(x-y) \\
& -\frac{i}{24 \pi}\left(-1+12 \pi Q^{2}\right) b^{m}(x-y)
\end{aligned}
$$

onde $Q$ neste caso é igual a $Q^{0}$ (componente correspondente ao campo $r^{0}$ que possui o termo cinético positiwo). De (3.101) e (3.108) notamos que a contribuiçäo de $T^{(1)}$ para 0 termo central é dada por 


$$
-i \frac{Q^{2}}{2}
$$

Redefinindo o vácuo de acordo com (3.106) verificamos que não há inversão de sinal no termo central. Invertendo o sinal da energia cinética verificamos uma inversão de sinal apenas do termo de improvement (apêndice E).

Resumimos os resultados analisados na tabela abaixo incluindo os sinais das normas em cada caso (veja apêndice E)

Campo Escalar com Termo de Improvement

\begin{tabular}{|c|c|c|c|c|}
\hline $\begin{array}{c}\text { Sinal } \\
\text { Energia } \\
\text { Cinética }\end{array}$ & $\begin{array}{c}\text { Definição } \\
\text { Vácuo }\end{array}$ & $\begin{array}{c}\text { Sinal } \\
\text { da } \\
\text { Norma }\end{array}$ & $\begin{array}{c}\text { Autovalores da } \\
\text { Hamiltoniana }\end{array}$ & $\begin{array}{c}\text { Termo Central } \\
\text { em Unidades de } \\
(-i / 24 \pi)\end{array}$ \\
\hline+ & $a \mid 0>=0$ & + & $E \geq 0$ & $1+12 \pi Q^{2}$ \\
\hline+ & $a^{\dagger} \mid 0>=0$ & - & $E \leq 0$ & $-1+12 \pi Q^{2}$ \\
\hline- & $a \mid 0>=0$ & - & $E \geq 0$ & $1-12 \pi Q^{2}$ \\
\hline- & $a^{\dagger} \mid 0>=0$ & + & $E \leq 0$ & $-1-12 \pi Q^{2}$ \\
\hline
\end{tabular}

É importante observar que $Q$ corresponde a $Q^{0}$ para o campo escalar com energia cinética positiva e a $Q^{1}$ para o campo escalar com energia cinética negativa.

Dos resultados analisados nesta seção e na anterior concluimos que o tensor total da soma dos campos e dos fantasmas satisfaz a álgebra

$$
\left[: T_{++}^{c}:+: T_{++}^{F}:,: T_{++}^{c}:+: T_{++}^{F}:\right]=-i\left(: T_{++}^{c}:+: T_{++}^{F}:\right)-\frac{i}{24 \pi}\left(c_{\varphi_{+}}+c_{\varphi_{-}}+c_{F}\right)
$$

onde : $T_{++}^{c}:$ e : $T_{++}^{F}:$ correspondem respectivamente as contribuições dos campos e fantasmas. $c_{\varphi_{+}}$e $c_{\varphi_{-}}$correspondem respectivamente aos valores do termo central para os campos com energia cinética positiva e energia cinética negativa, e $c_{F}$ corresponde ao termo central dos fantasmas. 
Das tabelas da seçäo anterior e desta seçäo concluimos que para o anulamento do termo central devemos ter [15]

$$
c_{Q_{+}+}+c_{p-}+c_{F}=-12 \pi Q_{0} Q^{a} \pm 20=0
$$

$\log 0$

$$
Q_{a} Q^{\mathrm{n}}= \pm \frac{13}{6 \pi}
$$

Portanto, neste caso tamos $Q_{0} Q^{a} \neq 0$.

O anulamento da carga central nestes casos ocorre para as saguintes definiçōes do vácuo

$$
\begin{aligned}
& a^{+}\left|0>=\left(a^{-}\right)^{\dagger}\right| 0>=b|0>=c| 0>=0 \\
& a^{+}\left|0>=\left(a^{-}\right)^{\dagger}\right| 0>=b^{\dagger}\left|0>=c^{\dagger}\right| 0>=0,
\end{aligned}
$$

onde $\left(a^{-}\right)^{\dagger}$ e $a^{4}$ correspondem respectivamente a operadores de criaçào para o campo escalar com energia cinética negativa e de aniquitaçäo para o campo escalar com energia cinética positiva, onde a primeira solução torresponde à solução com sinal positivo e segunda soluçāo com sinal negativo. Ambas as soluçôtes sĩo consistentes pois om ambos os casos o espaço de Hilbert para os campos escalarea possui norma positiva e para os fantasmas, que nằ possuem significado físico, possui norma nula.

Jackiw [15] comenta outras possibilidades para anular a anomalia, como usar operadores de aniquilação com energia positiva e as de criaçăo com energia negativa.

Conforme comentamos anteriormente, a soluçăo em (3.122) (3.123) (3.124) corresponde ao caso em que $Q^{a} Q_{a} \neq 0$ (ou seja, não temos o anulamento do temo central clássico). Varnos agora considerar o caso em que $Q^{2} Q_{a} \neq 0$ e desprezamos a contribuição dos Cantamas [34]. Este caso corresponde ao procedimento de quantizafáo canönico de operadores e não ao procedimento de quantizaçăo de BRST-BFV. Para o anulamento do termo central, devemos ter

$$
Q^{a} Q_{a}=\mp \frac{1}{6 \pi}
$$


Da tabela da página 51, verificamos que neste caso podemos ter as seguintes possibilidades para o vácuo

$$
\begin{aligned}
\left(a^{+}\right)^{\dagger} \mid 0> & =\left(a^{-}\right)^{\dagger} \mid 0>=0 \\
a^{+} \mid 0> & =a^{-} \mid 0>>0
\end{aligned}
$$

Da tabela verificamos que em ambos os casos o espaço de Filbert do campo com energia cinética negativa possui norma negativa. Portanto esta solucāo nao corresponde a un caso fisico de interesse, uma ves que a positividade da noma no espaço de Hibert é comprometida. Se considerarmos a contribuiç̆o dos lantasmas, chegaremos a mesma conclusão, una vez que estes apenas alteram o valor da carga central.

Chegaremos a mesma conclusầ na seçäo 3.6 .3 qutando analisarmos a soluçä̀ na representaşầo de Schoodinger.

E interessante também considerar o caso en que näo tenos cargas de fundo (termo de improvenent), ou seja, $Q_{a}=0$. Acui desconsideranos a contribuiçăo dos fantasmas. Neste caso, das tabelas da página 47 , temos o anulamento da anomalia para a seguinte definişăo do vácuo

$$
a^{+10}>=\left(a^{-}\right)^{\dagger} 10>=0
$$

Este caso corresponde a soluçăo

$$
t_{T}=c_{4}+c_{m}=1-1=0
$$

onde $x^{2}$ corresponde a carga central total e $t^{*}$ e $t$ correspondem respectivamente as contribuiçoes de $a^{+}$e $\left(a^{-}\right)^{\dagger}$ para $c_{T}$.

No caso

$$
\left(a^{\frac{1}{3}}\right)^{t} \mid 0>=a^{-1} 10>=0
$$

temos tambérn o anulamento da anomalia, mas a norma é negativa e esta soluçäo é desconsiderada. 
No caso em que năo consideramos a contribuiçăo dos fantasmas, ou seja, desconsideramos o método de quantizaçào de BRST-BFV e considerarnos $Q_{a} Q^{n}=0$, temos ainda o anulamento da anomalia para o caso

$$
a^{+}\left|0>=\left(a^{-}\right)^{\dagger}\right| 0>=0
$$

Conforme comentamos anteriomente existem na literatura outras formas de anular a anomalia. Básicamente existem três formas para atingir o anulamento da anomalia. A primeira, consiste em utilizar uma escolha nảo convencional para o vácuo. Iato significa que para alguns campos, os operadores de criação e aniquilaçăo possuem papéis invertidos. A segunda forma consiste en modificar os vínculos introduzindo termos colocados a mâa. A terceira consiste em introduzir cargas de fundo (introdugäo de um termo de improvennent). Jackiw [15] diseute as três possibilidades. 


\section{3,6.3 Quantizaçăo de Dirac para o Modelo de CGHS}

Para quantizarmos um sistema com vínculos usando o procedimento proposto por Dirac [20] é necessário inicialmente identificar os vinculos de primeira e segunda classe. Descrevemos a seguir os casos possiveis.

No caso em que existem apenas vínculos de primeira classe o primeiro passo é converter os campos em operadores. Substituimos em seguida os parênteses de Poisson entre duas funçöes dos campos pelos comutadores ou anticomutadores entre os correspondentes operadores, ou seja

$$
\{A, B\} \rightarrow \frac{[A, B]_{ \pm}}{i}
$$

onde aqui usamos $[A, B]_{\ldots}$ para comutadores $e[A, B]_{i}$ para anticomutadors.

$O$ próximo passo é impor os vínculos de primeira classe, anjui denotados por $\phi_{i}$ como operadores atuando nos vetores de estado do sistema, ou seja

$$
\hat{\phi}_{i} \mid \varphi>=0
$$

onde $\hat{h}_{i}$ é o operador correzpondente ao vínculo clássico de primeira classe $\phi_{i}$ e $\mid \varphi>$ mepresenta um estado fisico. O passo final é implementar os operadore que satisfazem a (3.131) e (3.132).

No caso do modelo de CGrIS possuimos apenas vínculos de primeira classe. Vamos quantizar a teoria, inicialmente desconsiderando o temo de improvement $\left(Q_{a}=0\right)$. Assim temos

$$
\begin{aligned}
& {\left[f_{a}(x), \Pi^{b}(y)\right]=b_{a}^{b} b(x-y)} \\
& \Pi_{s}(x)=-\frac{\dot{b}}{b r^{2}(x)}
\end{aligned}
$$

Os vínculos (3.50) säo impostos na forma operatorial sobre ofuncional de onda $\Psi$

$$
-\frac{1}{2}\left[-\frac{2}{\Lambda} \frac{\delta^{2} \Psi}{\delta r^{4}(x) b r_{a}(x)}+\frac{\Lambda}{2} r^{u z}(x) r_{s}^{f}(x) \Psi\right]=0
$$




$$
\frac{b U}{d r^{a}}=0
$$

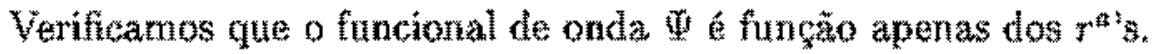

Agore resolvemos estas equaços juntamente com o operador hamiloniano aplicado a funçăo de onda. Conforme vimos na seçẫo 2.3 (equaçăo 2.53 ) a hamiltoniana é Identicam mente nula. Portanto, basta resolver (3.131).

Podemas verificar por substituiçă que temos duas solucöos que satisfazem (3.134)

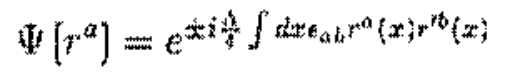

De fato, calculando a primeira e segunda derivadas de $\Psi$, obtemos

$$
\begin{aligned}
\frac{\delta \Psi}{\delta r^{a}} & = \pm i \frac{\Lambda}{2} \epsilon_{a b} r^{b} \Psi \\
\frac{\delta^{2} \Psi}{\delta r^{a} \delta r_{a}} & =\frac{\Lambda^{2}}{4} r_{a}^{\prime} r^{r a} \Psi
\end{aligned}
$$

e substituindo em (3.134), conchumos que os vinculos säo satisfeitos. Nas duas soluçōes encontradas a hamiltoniana possui autovalores mulos.

A soluçăo obtida para $\Psi$ esta de acordo com o resultado calculado por Jackiw [15].

Un sistema vinculado pode tambern ser quantizado utilizando-ge uma versäo diferente do procedimento de Dirac. Este é o método de quantiacăo do espaço de fase reduzido (RPS) [14]. No metodo proposto por Dirac, conforme vimos acima quantizamos o sistema primeiro e depois resol vemos os vinculos. No metodo RPS, primeiro resolvenos os víneulos e depois quantizamos o sistema.

Quantizamos a seguir o nodelo de CGHS usando o método RPS. Resolvendo os vintultos $(3.50)$ para o momento, obtemos

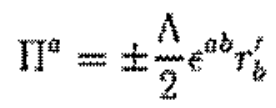

Logo operatorialmente devenos ter

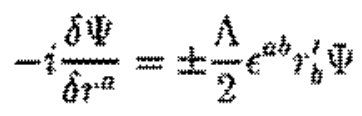


Esta equação é satisfeita pelas duas soluçōes encontradas para $\Psi$ (3.135) usando o procedimento usual de quantização de Dirac. De fato, usando a equaçāo (3.136) para a derivada primeira, obtemos uma identidade.

Se incluirmos o termo de improvement obtemos segundo (3.50) e (3.112)

$$
\begin{aligned}
& G_{0}=-\frac{1}{2}\left(\frac{2}{\Lambda} \Pi_{a} \Pi^{a}+\frac{\Lambda}{2} r_{a}^{\prime} r^{a \prime}\right)+\sqrt{\frac{\Lambda}{2}} Q_{a} r^{a \prime \prime} \\
& G_{1}=\Pi_{a} r^{a \prime}-\sqrt{\frac{2}{\Lambda}} Q_{a} \Pi^{a \prime}
\end{aligned}
$$

A funcional de onda deve portanto satisfazer a

$$
\begin{aligned}
-\frac{1}{2}\left(-\frac{2}{\Lambda} \frac{\delta^{2}}{\delta r^{a} \delta r_{a}}+\frac{\Lambda}{2} r_{a}^{\prime} r^{a \prime}\right) \Psi+\sqrt{\frac{\Lambda}{2}} Q_{a} r^{a \prime \prime} \Psi & =0 \\
r^{\alpha \prime} \frac{\delta \Psi}{\delta r^{a}}-\sqrt{\frac{2}{\Lambda}} Q_{a} \partial_{1} \frac{\delta \Psi}{\delta r^{a}} & =0
\end{aligned}
$$

obtemos as soluçōes

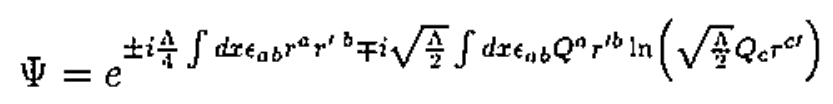

onde usamos (3.114).

Neste caso, como no caso anterior, verificamos que a solução satisfaz os vínculos usando as expressōes para as derivadas abaixo

$$
\begin{aligned}
\frac{\delta \Psi}{\delta r^{a}} & =\left[ \pm \frac{i}{2} \frac{\Lambda}{2} \epsilon_{a b} r^{b \prime} \pm i \sqrt{\frac{\Lambda}{2}} \frac{\epsilon_{d a} Q^{d} Q_{c} r^{c t}}{Q_{c} r^{c^{\prime}}}\right] \Psi \\
\frac{\delta^{2} \Psi}{\delta r^{a} \delta r_{a}} & =\frac{\Lambda^{2}}{4} r_{a}^{\prime} r^{a \prime} \Psi-2\left(\frac{\Lambda}{2}\right)^{3 / 2} Q_{a} r^{a \prime \prime} \Psi
\end{aligned}
$$

De forma semelhante ao caso anterior os autovalores da hamiltoniana são nulos. As soluçōes apresentadas em (3.141) sāo novas [34]. 
É interessante obter o funcional de onda do vácuo na representação de Schröedinger. Consideremos os casos $Q_{a}=0$ e $Q_{a} Q^{a}=0$. Utilizamos a expansāo de Fourier para o campo escalar (3.105)

$$
\varphi=\int_{-\infty}^{+\infty} d k \frac{1}{\sqrt{4 \pi|k|}}\left(a(k) e^{i k x}+a^{\dagger}(k) e^{-i k x}\right)
$$

Para o momento associado ao campo escalar com energia positiva, obtemos

$$
\pi=-i \int_{-\infty}^{+\infty} d k|k| \frac{1}{\sqrt{4 \pi|k|}}\left(a(k) e^{i k x}-a^{\dagger}\langle k) e^{-i k x}\right)
$$

Invertendo as equações acima, obtemos para $a(k)$ e $a^{\dagger}(k)$

$$
\begin{aligned}
a(k) & =\frac{1}{\sqrt{4 \pi|k|}} \int d x e^{-i k x}(|k| \varphi(x) \pm i \pi(x)) \\
a^{\dagger}(k) & =\frac{1}{\sqrt{4 \pi|k|}} \int d x e^{i k x}(|k| \varphi(x) \mp i \pi(x)),
\end{aligned}
$$

onde o sinal positivo é usado para o campo com energia cinética positiva $r^{0}$ e o sinal negativo para o campo com energia cinética negativa $r^{1}$. Conforme vimos anteriormente, a soluçāo de Fock no vácuo deve satisfazer a

$$
\begin{array}{r}
a^{+}(k) \Psi_{v}=0 \\
\left(a^{-}\right)^{\dagger}(k) \Psi_{y}=0
\end{array}
$$

onde $\Psi_{v}$ corresponde ao funcional de Schröedinger para o vácuo e onde $a^{+}$e $\left(a^{-}\right)^{\dagger}$ são respectivamente, os campos de aniquilaçào para o campo escalar com energia cinética positiva e criação para o campo escalar com energia cinética negativa.

Operatorialmente, as equações (3.145) podem ser escritas em termos de $r^{0}$ e $r^{1}$ como

$$
\int d x e^{-i k x}\left(|k| r^{0}(x)+\frac{\delta}{\delta r^{0}(x)}\right) \Psi_{v}=0
$$




$$
\int d x e^{i k x}\left(|k| r^{1}(x)+\frac{\delta}{\delta r^{1}(x)}\right) \Psi_{v}=0
$$

Por substituição, mostramos que a solução $\Psi_{v}$ é dada por

$$
\Psi_{v}=N e^{-\frac{1}{2} \int d x d y\left(r^{0}(x) w(x-y) r^{0}(y)+r^{1}(x) w(x-y) r^{1}(y)\right)},
$$

onde $\mathrm{N}$ é uma constante de normalização e onde

$$
w(x-y)=\frac{1}{2 \pi} \int d k|k| e^{i k(x-y)}
$$

$\mathrm{N}$ é calculada a partir da condição de normalização

$$
\int d r^{0} d r^{1} \Psi_{v} \Psi_{v}^{*}=1
$$

Obtemos

$$
N=\left(\frac{\operatorname{detw}}{\pi}\right)^{\frac{1}{2}}
$$

Logo

$$
\Psi_{v}=\left(\frac{\operatorname{det} w}{\pi}\right)^{\frac{1}{2}} e^{-\frac{1}{2} \int d x d y\left(r^{0}(x) w(x-y) r^{0}(y)+r^{1}(x) w(x-y) r^{1}(y)\right)}
$$

Agora verificaremos o caso em que $Q_{a} Q^{a}=\mp 1 / 6 \pi$. A solução que satisfaz a (3.126) é dada por

$$
\bar{\Psi}_{v}=e^{-\frac{1}{2} \int d x d y\left(r^{\circ}(x) w(x-y) r^{0}(y)-r^{1}(x) w(x-y) r^{1}(y)\right)}
$$

Esta solução é não-normalizável pois o expoente é indeterminado no termo em $r^{0}$ e não existe um estado de vácuo neste caso.

É interessante comentar neste ponto, um procedimento alternativo ao realizado neste trabalho e adotado por Kuchaï [35]. Ele introduz diretamente correções nos vínculos 
para eliminação da anomalia. Não procederemos neste trabalho com uma solução igual a de Jackiw, entretanto é interessante discutir alguns detalhes nos táleulos envolvendo o ordenamento normal em cada temo dos vínculos.

O produto normal de dois campos arbitrátios $A(x)$ e $B(y)$ é definido por

$$
: A(x) B(y):=A(x) B(y)-<0|A(x) B(y)| 0>
$$

Utilizando as expansōes em operadores de criaçäo e aniquilaçăo anteriores para os campos escalares, podemos escrever

$$
\begin{array}{r}
: \phi^{\prime}(x) \phi^{x}(y):=\phi(x)^{y} \phi(y)^{\prime} \pm \frac{1}{2} w(x-y) \\
: \pi(x) \pi(y):=\pi(x) \pi(y) \pm \frac{1}{2} w(x-y) \\
: \phi^{\prime}(x) \pi(y):=\phi^{\prime}(x) \pi(y)-\frac{i}{2} \delta^{\prime}(x-y),
\end{array}
$$

onde os sinais \pm são escolhidos de acordo com a tabela abaixo

\begin{tabular}{|c|c|c|}
\hline $\begin{array}{c}\text { Sinal-Energia } \\
\text { Cinética }\end{array}$ & Vácuo & $\begin{array}{c}\text { Sinal } \\
\text { Expressäo }\end{array}$ \\
\hline+ & $a \mid 0>=0$ & + \\
\hline+ & $a^{\dagger} \mid 0>=0$ & - \\
\hline- & $a \mid 0>=0$ & - \\
\hline- & $a^{\dagger} \mid 0>=0$ & + \\
\hline
\end{tabular}

Utilizando (3.139) e as equaçöes acima, obtemos para os vínculos

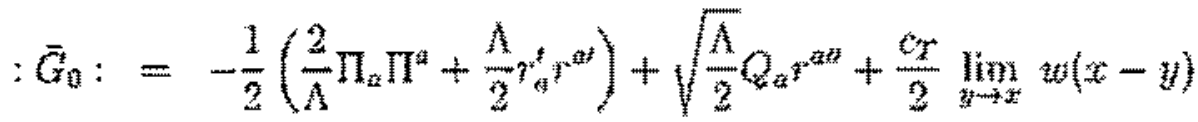

$$
\begin{aligned}
& : \bar{G}_{1}:=\Pi_{a} r^{* t}-\sqrt{\frac{2}{\Lambda}} Q_{a} \Pi^{a t}-i \lim _{y \rightarrow x} \delta(x-y)
\end{aligned}
$$


onde $c_{T}=0, \pm 2$, o lírnite e tomado para definir a fungäo no ponto $x$.

Agora as equaçöes que fornecem os estados fístcos na representaçāo de Schröedinger sẵo dadas por

$$
\begin{aligned}
& r^{a}(x) \frac{\partial \Psi}{\partial y^{a}(x)}+Q^{a}\left(\frac{\partial \Psi}{\partial t^{a}(x)}\right)^{\prime}+a \lim _{y \rightarrow z^{\prime}} \partial^{\prime}(x-y) \Psi=0
\end{aligned}
$$

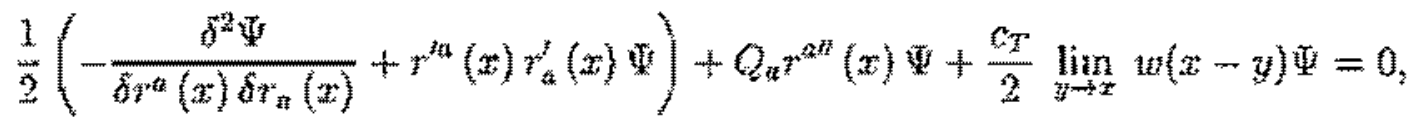

onde a buma constante que seleciona a prestic to de ordenamento dos operadores. A coolha mais simplatas e tomat $\alpha=0$.

Com a obtençäo dos funcionais de onda, completamos a quantizaça da teoria de CGHS. O sucesso na quantizacăo de una teotia de gravitaça em tuas dimensōes proporciona uma perspectiva tara quantizar outos modelos simplificados a teoria am quatro dimansōes.

Confoum citamos durante o texto, outros antotes chegaram a resultados similares utilizando outras variacoes dos procedimentos usuats de quantizaçầo. Entre os principais (veja capítulo ), estäo Mikovic, Jackiw a Cangemt.

Mikovic [14], trabalha com o modelo de CGHS com acoplanento do campo gravitacional aos campos de matéria. Utiliza o procedimento de quantikação canônica. Para escrever os vínculos na forma quadrática, utiliza um método baseado na transformação Bäcklund. Para eliminar a anomalia incroduz corregöes nos vinculos, cue estão de acordo com os resultados obtidos neste trabalho através da utilizaça de um termo de improvement. Nos cálculos da anomalia escreve o tensor momento-energia definido no círculo. Miković estuda também, a quantizaçäo do modelo via BRST operatorial. Conclui que a unitariedade é estabelecida trabalhando no gauge do cone de luz. Conclui ainda, que buracos negros näo violam o princípio da unitariedade e que näo existe perda de informaçāo. Argumenta ainda, que a intormagäo escapa durante o processo de evaporaçāo.

Jackiw e Cangemi [15] realizam também un estudo semelhante utilizando o mesmo modelo analisado por Miković. Trabalham com os procedimentos de quantização canônica e de BRST operatorial. Acrescentam ao estudo discussöes sobre a redefiniçà to vácuo 
para eliminaçăo da anomalia, devido a presença de um campo com energia cinética negativa. Utilizam alternativamente correções colocadas a mäo nos vínculos para eliminar a anomalia. Obtém a álgebra do tensor momento-energia tanto na reta quanto no chrculo. Discutem ainda inversöes nos sinais da energia para operadores de aniquilaçăo e criaçäo para eliminaçăo da anomalia.

Conforme, exposto no texto utilizamos nos cálculos uma composição do procedimento de quantizaçăo BRST.BFV e do procedimento de quantizaçăo canônica. Chegamos a resultados que estão de acordo com os de Miković, Jackiw e Cangemi. Tambếm cheganos a soluçōes novas no caso em que $Q_{0} Q^{a}$ é igual a zero. 


\section{Capítulo 4}

\section{Conclusão}

Aralisamos o modelo de CGHS usando o formalismo hamiltoniano e mostramos que esta teoria só possui vínculos de primeira classe. Usamos a técnica de BRST-BFV para quantizar a teoria. Mostramos que a função de partição pode ser integrada nos fantasmas que estẫo desacoplados dos campos. Utilizamos uma transformação canônica adequada para escrever os vínculos na forma quadrática, que permite obter o espaco de Hilbert. Verificemos que após asta transfornaça, a teoria inicial que possuia dois campos, o gravitacional e o campo dilatônico, é colocada na forma de uma teotia escalar com dós campos com assinaturas opostas. Vtilizamos o procedimento de quantização de Dirac para obter a álgebra de vínculos na forma do tensor momento-energia. Mostramos que existe uma anomalia ná álgebra. Mostramos que é possivel eliminar a anomalia acrescentando um termo de improvement a ação. Mostramos também um caso mais simples onde é possivel anular a anomalia utilizando o procedinento canônico, ou seja sem considerar a contribuiçăo de lantasmas introduzida pela técnica de BRST-BFV e sem considerar o termo de improvement. Encontramos soluçōes para a funçäo de onda atilizando o procedimento de Dirac. Mostramos ser possivel obter soluções físicamente viáveis nos casos em que as cargas de fundo $Q_{a}$ săo nulas e no caso em que st̃o diferentes de zero com $Q_{a} Q^{a}$ igual a zero. Estas últimas soluçōes não são encontradas na literatura. Acrescentamos uma discussão para o caso em que $Q_{a} Q^{a}=\mp 1 / 6 \pi$. Mostramos que neste caso a solução é insconsistente fisicamente pois possui norma negativa. Nestes casos em que a norma negativa é possivel mostrar atraves do funcional de onda na representaçäo de Schröedinger que a soluçăo é näo normalizável. Com isso provamos que o modelo de CGHS em $2 \mathrm{~d}$ pode 
ser quantizado utilizando-se a técnica de BRST-BFV.

Comparamos os resultados dos cálculos con os de outros autores, principalmente os de Mikovie, Jackiw e Cangemi que trabalharam em linhas semelhantes. Verificamos um perfeito acordo entre nossos resultados e os dessec autores.

A. quantizaçâo de uma teoria gravitacional em $2 \mathrm{~d}$ mostra um avanço no sentido de entender a quantizaçăo da teoria em $4 \mathrm{~d}$, que ainda não foi realizada. Isto mostra a importância dos modelos en $2 \mathrm{~d}$ e justifica estudos futuros incluindo o acoplamento de campos de matếria ao campo gravitacional para o estudo de formaçäo de buracos negros e confirmação das hipoteses de Hawking concernentes a evaporaçäo de buracos negros. 


\section{Apêndice A}

\section{Parênteses de Poisson}

Neste apêndice generalizamos as definiçōes e propriedades dos parênteses de Poisson para induirmos variáyeis de Grassmann no sistema estudado. Neste tratamento representaremos por $\varphi_{i}$ e $P^{i}$ os campos canônicamente conjugados do tipo bosônico e por ho $_{\text {ta }}$ e $\pi^{\alpha}$ os do tipo fermínico.

As definiçoes dos parênteses de Poisson entre campos bosônicos e fermionnicos devem satisfazer as condiçöes da mecânica quântica quando convertemos PB's am conutadores ou anticomuadores [28] [30] ou seja, os PB's entre dois campos bosonicos ou entre um campo bosônico e um fermồnico devem respeitar a propriedade de anticomutatividade e as PB's entre dois campos fermiônicos devem respeitar a propriedade comutativa. Rapresentando de maneira geral uma funçào bosônica dos campos por $\mathrm{E}$ e uma fermiônica por $F$ obtemos

$$
\begin{aligned}
& \left\{E_{1}, E_{2}\right\}=-\left\{E_{z}, E_{1}\right\}=\int d x\left[\left(\frac{\partial E_{1}}{\partial \varphi_{i}} \frac{\partial E_{2}}{\partial P^{i}}-\frac{\partial E_{2}}{\partial \varphi_{i}} \frac{\partial E_{1}}{\partial P^{i}}\right)+\left(\frac{\partial E_{1}}{\partial \psi_{\alpha}} \frac{\partial E_{2}}{\partial \pi^{\alpha}}-\frac{\partial E_{2}}{\partial \psi_{\alpha}} \frac{\partial E_{1}}{\partial \pi^{\alpha}}\right)\right] \\
& \left\{F_{1}, E\right\}=-\{E, F\}=\int d x\left[\left(\frac{\partial F}{\partial \varphi_{i}} \frac{\partial E}{\partial P^{i}}-\frac{\partial E}{\partial \varphi_{i}} \frac{\partial F}{\partial P^{i}}\right)-\left(\frac{\partial F}{\partial \psi_{\alpha}} \frac{\partial E_{\alpha}}{\partial \pi^{\alpha}}+\frac{\partial E}{\partial \psi_{\alpha}} \frac{\partial F}{\partial \pi^{\alpha}}\right)\right] \\
& \left\{F_{1}, F_{2}\right\}=\left\{F_{2}, F_{1}\right\}=\int d x\left[\left(\frac{\partial F_{1}}{\partial \varphi_{i}} \frac{\partial F_{2}}{\partial P^{i}}+\frac{\partial F_{2}}{\partial \varphi_{i}} \frac{\partial F_{1}}{\partial P^{i}}\right)-\left(\frac{\partial F_{1}}{\partial \psi_{\alpha k}} \frac{\partial F_{2}}{\partial \pi^{\alpha}}+\frac{\partial F_{2}}{\partial \psi_{\alpha}} \frac{\partial F_{1}}{\partial \pi^{*}}\right)\right]
\end{aligned}
$$

Rapresentando os campos de paridade arbitrária e sets respectivos momentos por $\psi_{7}$ e $\Pi^{y}$ podemos escrever as expressôes acima de forma mais geral como 


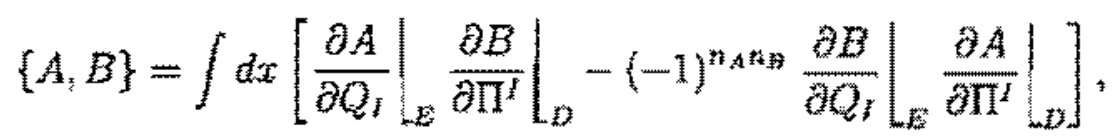

onde $n_{A}$ e $n_{E}$ fornecem a paridade das fungoes consideradus o os indices inferiones nas barras $\mathrm{E}$ e $\mathrm{D}$ indicam derivadas a esquerda e a direita respectivamente.

Os parêteses de Poisson generalizados saticfagen as seguntes propriedades

$$
\begin{gathered}
\{A, B\}=-(-1)^{n_{A} n_{B}}\{B, A\} \\
\{A, B+C\}=\{A, B\}+\{A, C\} \\
\{A, B C\}=\{A, B\}+(-1)^{n_{A} n_{B}} B\{A, C\} \\
(-1)^{n_{A} n_{B}}\{A,\{B, C\}\}+(-1)^{n_{B} n_{i 1}}\{B,\{C, A\}\}+(-1)^{n_{C} n_{A}}\{C,\{A, B\}\}=0
\end{gathered}
$$




\section{Apêndice B}

\section{Tensor Momento Energia para os}

\section{Fantasmas}

Vamos inicialmente escrever a ação para os fantasmas á partir da equaçāo (3.96) escrita na forma covariante

$$
L=-\frac{1}{2} \int d x \sqrt{-g} g^{\mu \nu} c^{\rho} D_{\mu} b_{\nu \rho}
$$

Temos

$$
D_{\mu} b_{\nu \rho}=\partial_{\mu} b_{\nu \rho}-\Gamma_{\mu(\nu}^{\lambda} b_{\rho) \lambda}
$$

A variação da ação com relação a métrica é dada por

$$
\begin{aligned}
\delta S & =-\frac{1}{2} \int d^{2} x \sqrt{-g}\left\{-\frac{1}{2} \delta g^{\alpha \beta} g_{\alpha \beta} g^{\mu \nu} c^{\rho} D_{\mu} b_{\nu \rho}+\delta g^{\mu \nu} c^{\rho} D_{\mu} b_{\nu \rho}\right. \\
& \left.-g^{\mu \nu} c^{\rho} \frac{1}{2} g^{\lambda \sigma}\left(D_{\mu} \delta g_{(\nu \sigma}+D_{(\nu} \delta g_{\mu \sigma}-D_{\sigma} \delta g_{\mu(\nu}\right) b_{\rho) \lambda}\right\}
\end{aligned}
$$

onde usamos [33]

$$
\delta \Gamma_{\mu \nu}^{\lambda}=\frac{1}{2} g^{\lambda m}\left[D_{\nu} \delta g_{m \mu}+D_{\mu} \delta g_{m \nu}-D_{m} \delta g_{\mu \nu}\right]
$$


O último termo de $(B, 3)$ pode ser escrito como

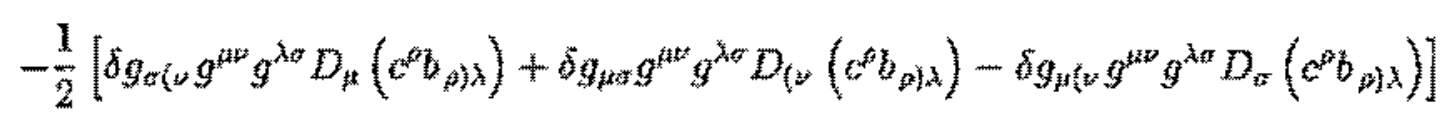

ou desenvolvendo

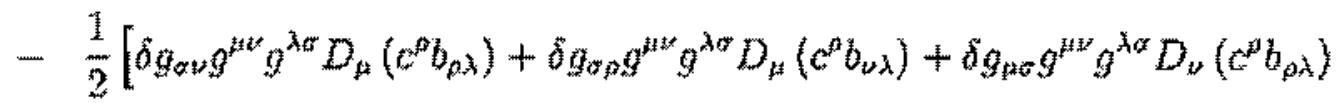

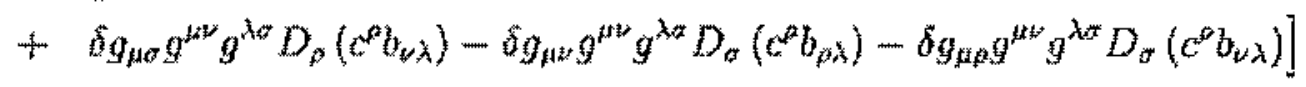

e simplificando

$$
-\delta g_{\mu \sigma} g^{\mu \nu} g^{\lambda \sigma} D_{\nu}\left(c^{\rho} b_{\rho \lambda}\right)-\frac{1}{2} \delta g_{\mu \sigma \alpha} g^{\mu \nu \mu} g^{\lambda \sigma} D_{\rho}\left(c^{\rho} b_{\nu \lambda \lambda}\right)+\frac{1}{2} \delta g_{\mu \nu} g^{\mu \omega} g^{\lambda \sigma} D_{\sigma}\left(c^{p} b_{p \lambda}\right)
$$

Substituindo o último termo simplificado na expressão (B.3)

$$
\begin{aligned}
& \delta S=-\frac{1}{2} \int d^{2} x \sqrt{-g} \delta g^{\alpha \beta}\left\{-\frac{1}{2} g_{\alpha \beta} g^{\mu \alpha i} c^{\rho} D_{\mu \alpha} b_{\nu \rho}+c^{p} D_{\alpha} b_{\beta p}-D_{\beta}\left(c^{\rho} b_{\beta \alpha}\right)\right. \\
& \left.-\frac{1}{2} D_{p}\left(c^{p} b_{\alpha \beta}\right)+\frac{1}{2} g_{\alpha \beta g^{\lambda \alpha}} D_{\alpha}\left(c^{p} b_{p \lambda}\right)\right\}
\end{aligned}
$$

desenvolvendo o terceiro termo e agrupando o primeiro e cuinto temos, temos

$$
\begin{aligned}
& \delta S=-\frac{1}{2} \int d^{2} x \sqrt{-g} \delta g^{\alpha \beta}\left\{-D_{g} c^{p} b_{p a}-\frac{1}{2} D_{p} c^{p} b_{a b}-\frac{1}{2} c^{p} D_{p} b_{\alpha} s\right. \\
& \left.-\frac{1}{2} g_{\alpha a}\left(g^{\mu \nu} c^{p} D_{\mu} b_{\nu p}-g^{\lambda o} D_{\alpha} c^{p} b_{p \lambda}-g^{\lambda \mu r} c^{p} D_{q} b_{p \lambda}\right)\right\}
\end{aligned}
$$

Simplificando

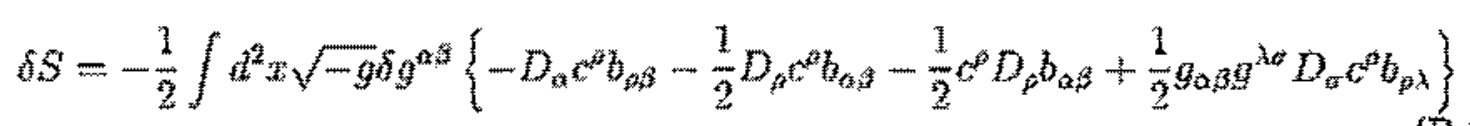

e usando a relaçãa 


$$
\delta\left(g^{\mu \nu} b_{\mu \nu}\right)=\delta\left(b_{\mu}^{\mu}\right)=\delta g^{\mu t} b_{k \mu \nu}=0
$$

obtemos

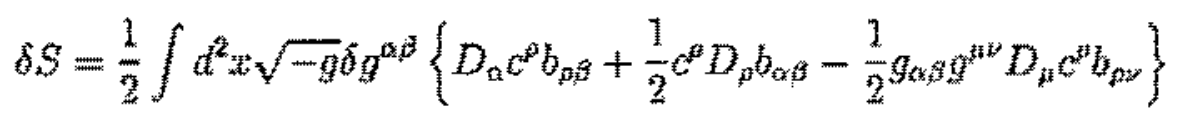

Portante obtemos para T

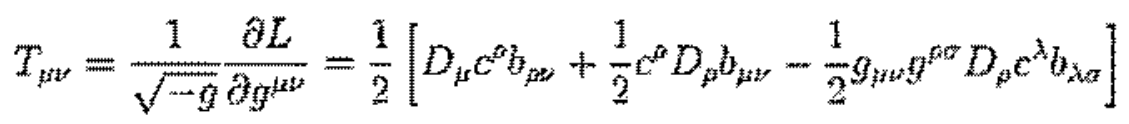

ou

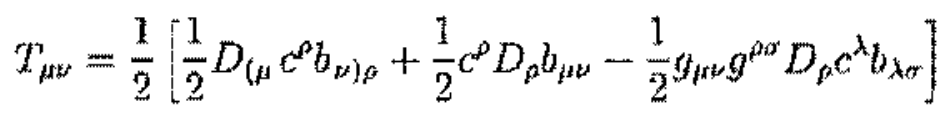

Para escrever as componentes do tensor momento-energia nas coordenadas do cone de luz, vamos usar as equax̧óes de movimento obtidas a partir da lagrangeaña (3.06). A acāo para os fantasmas é escrita como

$$
S=-\frac{1}{2} \int d^{2} x c^{*} \partial^{*} b_{3 *}
$$

ou en temos de componentes nas coordenadas do cone de luz

$$
s=-\frac{1}{2} \int d x\left(c^{+} \partial^{+} b_{++}+c^{-} \partial^{-} b_{m--}\right)
$$

ou

$$
L=\frac{1}{2} \int d x\left(c^{+} \partial_{-} b_{+}+c^{-y_{+} b_{--}}\right) .
$$

onde usamos o fato de que $b_{+-}=0$ é obtido de

$$
b_{\mu}^{\mu}=n^{\mu \nu} b_{\mu \nu}=2 n^{+-} b_{+-}=0
$$

onde $\eta^{t / 2}$ representa a métrica de Minkowski.

De (B.17) obtemos as equaçōe de movimento respectivamente para $c^{4}$ e $c^{m}$ 


$$
\begin{aligned}
& \partial_{-} b_{++}=0 \\
& \partial_{+} b_{-}=0
\end{aligned}
$$

e respectivamente para $b_{++}$e $b_{--}$

$$
\begin{aligned}
& \partial_{-} c^{+}=0 \\
& \partial_{+} c^{-}=0
\end{aligned}
$$

Escrevenos agora (B.14) nas coordenadas do cone de luz usando as equarçoes de movimento acima obtemos

$$
\begin{aligned}
& T_{ \pm \pm}=\frac{1}{2} \partial_{ \pm} c^{ \pm} b_{ \pm}+\frac{1}{4} c^{ \pm} \partial_{ \pm} b_{ \pm 2} \\
& \tau_{+}=0
\end{aligned}
$$




\section{Apêndice $\mathrm{C}$}

\section{Álgebra do Tensor Momento-Energia para o Campo Escalar}

A lagrangeana para o campo escalar com termo cinético positivo, é dado por (E.1)

$$
L=-\frac{1}{2} \int d x \partial^{\prime} \varphi \partial_{5} \varphi
$$

Usando (3.95), obtemos para o tensor momento-energia

$$
T_{ \pm \pm}^{(S)}=-\frac{1}{2} \partial_{ \pm} \varphi \partial_{i} \varphi
$$

Classicamente é facil mostrar que o tensor momento-energia satisfaz a álgebra

$$
\left\{T_{++}^{(S)}(x), T_{++}^{(S)}(y)\right\}=-\left(T_{++}^{(S)}(x)+T_{++}^{(S)}(y)\right)
$$

Quânticamente, a álgebra pode apresentar uma anomalia. Fazendo a análise dimensional da expresșào acima esperamos para a ălgebra quẩntica

$$
\left[: T_{++}^{(S)}(x):: T_{++}^{(S)}(y):\right]=-i\left(: T_{++}^{(S)}(x):+: T_{++}^{(S)}(y):\right) f^{y}(x-y)+c \delta^{z y}(x-y)
$$

onde "c" corresponde a contribuição do termo central. Este termo é conhecido como termo de Schwinger $[15]$. 
Vamos calcular o termo central expandido o campo $\varphi$ em operadores de aniquilação e criação

$$
\varphi(x)=\int_{-\infty}^{+\infty} \frac{d \mathbf{k}}{\sqrt{2 \pi}} \frac{1}{2|\mathbf{k}|}\left[a(\mathbf{k}) e^{i k x}+a^{\dagger}(\mathbf{k}) e^{-i k x}\right]
$$

onde

$$
k x=-|\mathbf{k}| x^{0}+\mathbf{k x}
$$

e onde usamos a convenção usual nas exponenciais, ou seja, " $a$ "” associado ao termo com energia positiva $\mathrm{e}$ " $a$ " ao termo com energia negativa.

$\mathrm{O}$ momento canônicamente conjugado a $\varphi$ é dado por

$$
\pi=\dot{\varphi}
$$

$\log 0$

$$
\pi=-\frac{i}{2} \int_{-\infty}^{+\infty} \frac{d \mathbf{k}}{\sqrt{2 \pi}}\left[a(\mathbf{k}) e^{i k x}-a^{\dagger}(\mathbf{k}) e^{-i k x}\right]
$$

Utilizando o procedimento de Dirac devemos ter

$$
[\varphi(x), \pi(y)]=i \delta(x-y)
$$

utilizando as expansões acima em (C.9), obtemos

$$
\left[a(\mathbf{k}), a^{\dagger}\left(\mathbf{k}^{\prime}\right)\right]=2|\mathbf{k}| \delta\left(\mathbf{k}-\mathbf{k}^{\prime}\right)
$$

Normalizando $a(\mathbf{k})$ para facilitar os cálculos

$$
a \longrightarrow \sqrt{2|\mathbf{k}|} a
$$

obtemos 


$$
\left[a(\mathbf{k}), a^{\dagger}\left(\mathbf{k}^{t}\right)\right]=\delta\left(\mathbf{k}-\mathbf{k}^{\prime}\right)
$$

E reeserevemos $p(x)$ e $\pi(x)$ como

$$
\begin{gathered}
\varphi(x)=\int_{-\infty}^{+\infty} \frac{d \mathbf{k}}{\sqrt{2 \pi}} \frac{1}{\sqrt{2|\mathbf{k}|}}\left[a(\mathbf{k}) e^{i k+}+a^{\dagger}(\mathbf{k}) e^{-i k x}\right] \\
n(x)=-\frac{i}{\sqrt{2}} \int_{-\infty}^{+\infty} \frac{d \mathbf{k}}{\sqrt{2 \pi}} \sqrt{|\mathbf{k}|}\left[a(\mathbf{k}) e^{i k x}-a^{\dagger}(\mathbf{k}) e^{-i k x}\right]
\end{gathered}
$$

A derivada $\partial_{+} \varphi(x)$ é duda por

$$
\partial_{+} \varphi(x)=-i \int_{-\infty}^{a} \frac{d \mathrm{k}}{\sqrt{2 \pi}} \sqrt{|\mathrm{k}|}\left[a(\mathrm{k}) e^{i k x}-a^{\dagger}(\mathrm{k}) e^{-m+k x}\right],
$$

onde utilizamos o intervalo de $(-\infty, 0)$ para facilitar os cálculos.

Vamos usar a substituiçăo $\mathrm{k} \rightarrow-\mathbf{k}$ em $\partial_{+}$para trabalharmos apenas com frequencias positivas. Para facilita a notaçäo definimos ainda

$$
\begin{aligned}
A(\mathbf{k}) & =a^{\ddagger}(-\mathrm{k}) \\
A^{\dagger}(\mathrm{k}) & =a(-\mathrm{k}) \\
{\left[A^{\dagger}(\mathrm{k}), A\left(\mathrm{k}^{\prime}\right)\right] } & =\left[a(-\mathrm{k}), a^{\dagger}\left(-\mathrm{k}^{\dagger}\right)\right]=\delta\left(\mathbf{k}-\mathrm{k}^{\dagger}\right) \\
A^{\dagger}(\mathrm{k}) \mid 0> & =a(-\mathrm{k}) \mid 0>=0 \\
\mathbf{k} & >0
\end{aligned}
$$

Com estas consideraços, e usando $t=0$ para facilitar os cullculos obtemos

$$
\partial_{+} \varphi(x)=-i \int_{0}^{+\infty} \frac{d \mathbf{k}}{\sqrt{2 \pi}} \sqrt{|\mathbf{k}|}\left[A^{\dagger}(\mathbf{k}) e^{-i \mathbf{k} x}-A(\mathbf{k}) e^{+i k x}\right]
$$

onde usamos $x$, en vez de $x$ para facilitar a notação.

Usando esta equaçăo em (C.2) obtém-se para $T_{++}^{(S)}(x)$ 


$$
\begin{aligned}
: T_{++}^{(S)}(x): & =\int_{0}^{+\infty} \int_{0}^{+\infty} \frac{d \mathbf{k} d \mathbf{k}^{\prime}}{4 \pi} \sqrt{\mathbf{k k}^{\prime}}\left[A^{\dagger}(\mathbf{k}) A^{\dagger}\left(\mathbf{k}^{\prime}\right) e^{-i\left(k+k^{\prime}\right) x}\right. \\
& \left.-2 A(\mathbf{k}) A^{\dagger}\left(\mathbf{k}^{\prime}\right) e^{-i\left(\mathbf{k}^{\prime}-k^{\dagger}\right) x}+A(\mathbf{k}) A\left(\mathbf{k}^{\prime}\right) e^{i\left(k+\mathbf{k}^{\gamma}\right) x}\right]
\end{aligned}
$$

Como "c" em (C.4) é um numero, o cálculo do termo central é feito através do elemento de matriz

$$
<0\left|\left[: T_{++}^{(S)}(x):, T_{++}^{(S)}(y)\right]\right| 0>
$$

Substituindo (C.18) en (C.19) obtemos

$$
\begin{aligned}
& <0\left|\left[: T_{++}^{(S)}(x):,: T_{++}^{(S)}(n):\right]\right| 0>=\int_{0}^{+\infty} \int_{0}^{+\infty} \int_{0}^{+\infty} \int_{0}^{+\infty} \frac{d \mathbf{k d} d \mathrm{k}^{\prime}}{4 \pi} \frac{d \mathrm{p} d \mathrm{p}^{\prime}}{4 \pi} \sqrt{\mathrm{kk}^{\prime}} \sqrt{\mathrm{pp}^{\prime}} \\
& <0\left|A^{\dagger}(\mathrm{k}) A^{\dagger}\left(\mathbf{k}^{\prime}\right) A(\mathbf{p}) A\left(\mathbf{p}^{\prime}\right)\right| 0>\left[e^{-i\left(k+\mathrm{k}^{\prime}\right) x} e^{i\left(\mathbf{p}+\mathbf{p}^{\prime}\right) y}-e^{-i\left(\mathrm{k}+\mathrm{k}^{\prime}\right) y} e^{i\left(\mathrm{p}+\mathrm{p}^{\prime}\right) \mathrm{y}}\right]
\end{aligned}
$$

usando $(\mathrm{C} .16)$

$$
\begin{aligned}
& <0\left|\left[: T_{++}^{(S)}(x):: T_{++}^{(S)}(y):\right]\right| 0>=\int_{0}^{+\infty} \int_{0}^{+\infty} \int_{0}^{+\infty} \int_{0}^{+\infty} \frac{d \mathbf{k} d \mathbf{k}^{\prime}}{4 \pi} \frac{d \mathrm{pdp}^{\prime}}{4 \pi} \sqrt{\mathbf{k k}^{\prime}} \sqrt{\mathrm{pp}^{\prime}}
\end{aligned}
$$

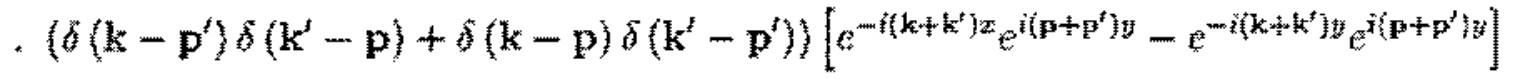

Integrando sobre $\mathrm{p}$ e $\mathrm{p}^{\prime}$

$$
<0\left|\left[: T_{++}^{(S)}(x):,: T_{++}^{(S)}(y):\right]\right| 0>=2 \int_{0}^{+\infty} \frac{d \mathbf{k} d \mathbf{k}^{\prime}}{(4 \pi)^{2}} \mathbf{k k}^{\prime}\left(e^{-i\left(\mathbf{k}+\mathbf{k}^{\prime}\right)(x-y)}-e^{i\left(\mathbf{k}+\mathbf{k}^{\prime}\right)(x-y)}\right)
$$

Usando a transformaçăo de varthảeis 


$$
\begin{aligned}
\int_{0}^{+\infty} d \mathbf{k} d \mathbf{k}^{\prime} f\left(\mathbf{k}, \mathbf{k}^{\prime}\right) & =\int_{0}^{+\infty} d q \int_{0}^{q} d q^{\prime} f\left(q-q^{\prime}, q^{\prime}\right) \\
\mathbf{k} & =q-q^{\prime} \\
\mathbf{k}^{\prime} & =q^{\prime}
\end{aligned}
$$

Obtemos

$$
<0\left|\left[: T_{++}^{(S)}(x):,: T_{++}^{(S)}(y):\right]\right| 0>=-\int_{0}^{+\infty} \frac{d q}{\delta \pi^{2}} \int_{0}^{q} d q^{\prime}\left(q-q^{\prime}\right) q^{\prime}\left(e^{i q(x-y)}-e^{-i q(x-y)}\right)
$$

on

$$
<0\left|\left[: T_{++}^{(s)}(x): ; T_{++}^{(s)}(y):\right]\right| 0>=-\frac{1}{48 \pi^{2}} \int_{-\infty}^{+\infty} d q q^{3} e^{i q(x-y)}=-\frac{i}{24 \pi} \delta^{t \prime \prime}(x-y)
$$

$\log 0$

$$
c=-\frac{i}{24 \pi}
$$

Vamos agora verificar o primeiro termo do lado direito de (C.4). O elemento de matríz a ser usado neste échlculo deve ser tal que permita reobter o tensor no primeiro termo do lado direito da expressäo para fechar a álgebra. Os cálculos a seguir mostram que o elentento de matriz adequado é o seguinte

$$
\left.<0\left|\left[: T_{++}^{(S)}(x)::: T_{++}^{(s)}(y)\right]\right| q_{1} q_{2}\right\rangle
$$

onde

$$
\left|q_{1} q_{2}>=A\left(a_{1}\right) A\left(q_{2}\right)\right| 0>
$$

Vsando (C.18) em (C.27) 


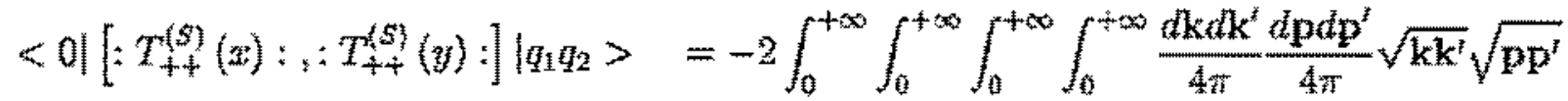

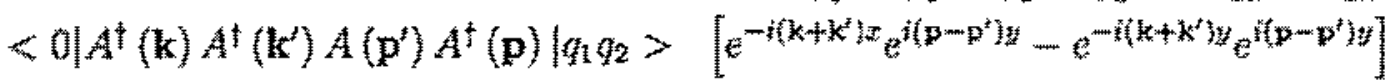

out

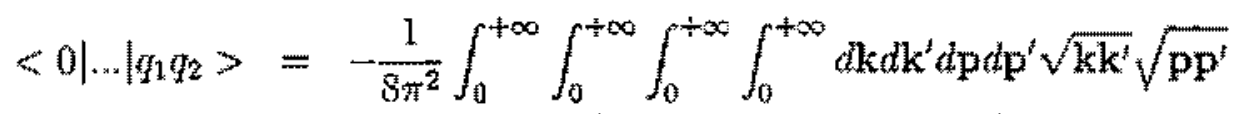

$$
\begin{aligned}
& \text { - }\left[\delta\left(\mathbf{k}^{\prime}-p\right) \delta\left(\mathbf{p}^{\prime}-q_{1}\right) \delta\left(\mathbf{k}-q_{2}\right)+\delta\left(\mathbf{k}^{2}-p\right) \delta\left(\mathbf{k}-q_{1}\right) \delta\left(\mathbf{p}^{\prime}-q_{2}\right)\right. \\
& \left.+\delta(\mathrm{k}-\mathrm{p}) \delta\left(\mathrm{p}^{t}-q_{\mathrm{n}}\right) \delta\left(\mathrm{k}^{t}-q_{2}\right)+\delta(\mathrm{k}-\mathrm{p}) \delta\left(\mathrm{k}^{t}-q_{\mathrm{t}}\right) \delta\left(\mathrm{p}^{t}-q_{2}\right)\right) \\
& \text {. }\left[e^{-i\left(k+k^{\prime}\right) x} e^{i\left(p-p^{\prime}\right) y}-e^{-i\left(k+k^{\prime}\right) y} e^{i\left(p-p^{\prime}\right) t y}\right],
\end{aligned}
$$

onde $<0|\ldots| g_{1} g_{2}>$ corresponde a $\left.<0\left|\left[: T_{++}^{(s)}(x):, T_{+}^{(\$)}(y):\right]\right| q_{1} q_{2}\right\rangle$.

Integrando sobre $\mathrm{k}^{i}, \mathrm{p}$ e $\mathrm{p}^{\prime}$

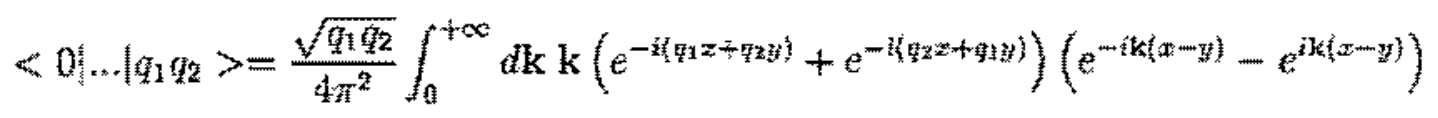

ou

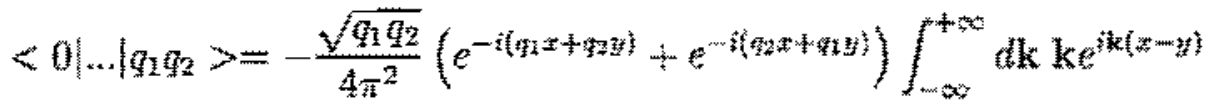

$$
\begin{aligned}
& <0|\ldots| q_{1} q_{2}>=-i \frac{\sqrt{q_{1} q_{2}}}{2 \pi}\left(e^{-i\left(q_{1}+q_{2} y\right)}+e^{-i\left(q_{2} x+q_{1} y\right)}\right) \delta^{\prime}(x-y)
\end{aligned}
$$

onde $\delta^{\prime}(x-y)$ corresponde $a \partial_{x} \delta(x-y)$. Podenos escrever (C.33) usando as identidades abaixa: 


$$
\begin{aligned}
& e^{-i q_{1} x-i q_{2} y} \delta^{\prime}(x-y)=e^{-i\left(q_{1}+q_{2}\right) x} \delta^{\prime}(x-y)-i q_{2} e^{-i\left(q_{1}+q_{ \pm}\right) x} \delta(x-y) \\
& e^{-i q_{2} x-i q_{2} y_{\delta^{\prime}}}(x-y)=e^{-i\left(q_{1}+y_{2}\right) y} \delta^{\prime}(x-y)+i q_{2} e^{i\left(q_{1}+q_{2}\right) y} \delta(x-y)
\end{aligned}
$$

Substituindo $(\mathrm{C} .34)$ e (C.35) e (C.33)

$$
<0|\ldots| q_{1} q_{2}>=-\frac{\sqrt{9 q_{2}}}{2 \pi}\left(e^{-i\left(q_{1}+q_{2}\right) x}+e^{-i\left(q_{1}+q_{2}\right) y}\right) b^{t}(x-\eta)
$$

Calculamos agora $<0\left|: T_{+}^{3}(x):\right| \omega_{1} q_{2}>$ para comparar com $(\mathrm{C} .30)$. Usando $(0.18)$

$$
\begin{aligned}
& \left.\left.<0\left|: T_{++}^{(S)}(x):\right| q_{\mathrm{r}} q_{2}\right\rangle=\int_{0}^{+\infty} \frac{d \mathbf{k} d \mathbf{k}^{\prime}}{4 \pi} \sqrt{\mathbf{k} \mathbf{k}^{\prime}}<0\left|A^{\dagger}(\mathbf{k}) A^{\dagger}\left(\mathbf{k}^{\prime}\right)\right| q_{1} q_{2}\right\rangle e^{-i\left(\mathbf{k}+\mathrm{k}^{\prime}\right) x} \\
& \left\langle 0\left|: T_{+1}^{(S)}(x):\right| q_{1} q_{2}\right\rangle=\int_{0}^{+\infty} \frac{d \mathbf{k} d \mathbf{k}^{\prime}}{4 \pi} \sqrt{\mathbf{k k}^{\prime}}\left[\delta\left(\mathrm{k}^{\prime}-q_{1}\right) \delta\left(\mathbf{k}-q_{2}\right)\right. \\
& \left.+\delta\left(\mathbf{k}-q_{1}\right) \delta\left(\mathbf{k}^{t}-q_{2}\right)\right] e^{-\mathrm{i}\left(\mathrm{k}+\mathrm{k}^{\prime}\right) \mathrm{k}}
\end{aligned}
$$

Integrando sobre $\mathbf{k}^{t} \mathbf{e} \mathbf{k}$

$$
\left.<0\left|: T_{+\rightarrow}^{(5)}(x):\right| q_{1} q_{2}\right\rangle=\frac{1}{2 \pi} \sqrt{q_{1} q_{2}} e^{-i\left(q_{2}+q_{2}\right) x}
$$

De (C.36) e (C.39) obtemos o prímeiro termo de (C.4).

Fica assim demonstrada a álgebra do censor $T_{++}^{(S)}$.

É interessante tambén discutir neste ponto, o sinal da norma no espaço de Hilbert. Levando em consideraçäo a relação de comutação dada em (C.16) obtemos

$$
<0\left|A(k) A^{\dagger}\left(k^{\prime}\right) 0\right|>=-\delta\left(k-k^{\prime}\right)<0 \mid 0>
$$

Logo neste caso temos uma norma negativa, o que não é consistente com a definiçăo do espaço de Hilbert onde requeremos noma positiva. 
Vamos analisar agora o efeito da mudança na definiçăo do vácuo sobre o termo central. Redefinimos o vácuo para

$$
A 10>=0
$$

Mudando o ordenamento de $: T_{++1}^{(S)}(x)$ : a expressano $(\mathrm{C} .18)$ toma agora a forma

$$
\begin{aligned}
: T_{++}^{(S)}(x): & =\int_{0}^{+\infty} \frac{d \mathbf{k} d \mathbf{k}^{\prime}}{4 \pi} \sqrt{\mathbf{k k}^{\prime}}\left[A^{\dagger}(\mathbf{k}) A^{\dagger}\left(\mathbf{k}^{\prime}\right) e^{-i\left(\mathbf{k}+k^{\prime}\right) x}\right. \\
& \left.-2 A^{\dagger}(\mathbf{k}) A\left(\mathbf{k}^{\prime}\right) e^{-i\left(\mathbf{k}-k^{\prime}\right) x}+A(\mathbf{k}) A\left(\mathbf{k}^{\prime}\right) e^{i\left(\mathbf{k}+\mathbf{k}^{\prime}\right) x}\right]
\end{aligned}
$$

e o termo central é agora escrito seguindo procedimento similar ao caso da definiçäo usual do vácuo como

$$
<0 \mid\left[: T_{+}^{(S)}(x):,: T_{++}^{(S)}(y): \mid 0>=2 \int_{0}^{+\infty} \frac{d \mathbf{k} d \mathbf{k}^{\prime}}{(4 \pi)^{2}} \mathbf{k k}^{\prime}\left(e^{i\left(\mathbf{k}+\mathbf{k}^{\prime}\right)(x-y)}-e^{-i\left(\mathbf{k}+\mathbf{k}^{\prime}\right)(x-y)}\right)\right.
$$

Comparando $(0.22) \mathrm{com}(\mathrm{C} .43)$ verificamos que existe uma inversão de sinal do termo central devido a mudança de sirual nas exponenciais. Logo

$$
c=\frac{i}{24 \pi}
$$

Portanto concluimos que a nova definição para o vácuo introduz uma inversäo de sinal do termo central.

Neste caso, temos

$$
<0\left|A^{\dagger}(k) A\left(k^{\prime}\right)\right| 0>=\delta\left(k-k^{\prime}\right)<0 \mid 0>
$$

Logo a norma é positiva.

Una vez que temos também um termo na ação com energia cinética negativa vamos analisar o caso (veja (3.73)) 


$$
L=\frac{1}{2} \int d x \partial^{\mu} \varphi \partial_{\mu} \varphi
$$

Agora o momento canônicamente conjugado a $\varphi$ é dado por

$$
\pi=-\dot{p}
$$

Para manter

$$
\{\varphi(x), \pi(y)\}=i \tilde{b}(x-y)
$$

devenos ter agora

$$
\begin{aligned}
{\left[A^{\dagger}(\mathbf{k}), A\left(\mathbf{k}^{\prime}\right)\right] } & =\left[a(-\mathbf{k}), a^{\dagger}\left(\cdots \mathbf{k}^{\prime}\right)\right]=-\delta\left(\mathbf{k}-\mathbf{k}^{\prime}\right) \\
A^{\dagger}(\mathbf{k}) \mid 0> & =0
\end{aligned}
$$

Temos, portento, : $T_{++}^{(S)}(x)$ : com sinal invertido para energia cinética negativa, mas o termo central mantém o mesmo sinal obtido en $(\mathrm{C} .25)$. De fato, o callculo envolve o produto de duas relaçōes do comutaçăo.

Assim

$$
c=\frac{i}{24 \pi}
$$

Neste caso obtemos o sinal para a norma da mesma forma que no caso anterior

$$
<0\left|A(k) A^{\dagger}\left(k^{t}\right)\right| 0>=\delta\left(k-k^{r}\right)<0 \mid 0>
$$

Assim, possuimos norma positiva.

Se redefinimos o vácuo para

$$
A(\mathbf{k}) 10>=0
$$


obtemos uma inversăo de sinal do termo central como no caso (C.44)

$$
c=\frac{i}{24 \pi}
$$

Neste caso temos

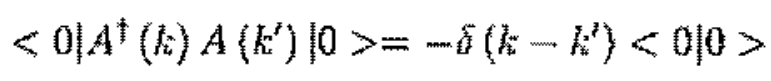

assim possuimos norma negativa.

Para analsamos os autovalores do operador hamiltoniano, no caso do campo escalar com anergia cinética positiva, usamos as expancōes para (C.13) \& $\pi(0.14)$ na expressão $(3.108)$, Obtemos

$$
\begin{gathered}
H=2 \int_{0}^{+\infty} d \mathbf{k ~ k} A^{\dagger}(\mathbf{k}) A(\mathbf{k}) \\
{\left[A^{+}(\mathbf{k}), A\left(\mathbf{k}^{\prime}\right)\right]=\delta\left(\mathbf{k}-\mathrm{k}^{\prime}\right)} \\
A(\mathbf{k}) \mid 0>=0
\end{gathered}
$$

Os autovalores E neste caso säo positivos, uma vez que a hamiltoniana é positiva definida, ou seja

$$
E \leq 0
$$

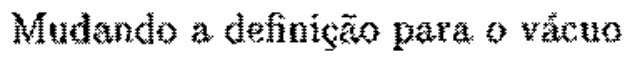

$$
\begin{gathered}
H=2 \int_{0}^{+\infty} d \mathrm{k} \mathrm{k} A(\mathrm{k}) A^{\dagger}(\mathrm{k}) \\
{\left[A^{\dagger}(\mathrm{k}), A\left(\mathrm{k}^{\prime}\right)\right]=\delta\left[\mathrm{k}-\mathrm{k}^{\prime}\right)} \\
A^{*}(\mathrm{k}) \mid 0>=0
\end{gathered}
$$

obtemos autovalores negativos, uma vez qque a hamiltoniana é negativa definida 


$$
E \geq 0
$$

Mudando para a lagrangeana escalar com energia cinética negativa o usando a definição usual para o vácuo

$$
\begin{aligned}
& H=-2 \int_{0}^{+\infty} d \mathbf{k} \mathbf{k} A^{\dagger}(\mathbf{k}) A(\mathbf{k}) \\
& {\left[A^{\dagger}(\mathbf{k}), A\left(\mathbf{k}^{\prime}\right)\right]=-\delta\left(\mathbf{k}-\mathbf{k}^{\prime}\right) } \\
& A(\mathbf{k}) \mid 0>=0 \\
& E \leq 0
\end{aligned}
$$

e mudando a definição para o vâcuo

$$
\begin{aligned}
& H=-2 \int_{0}^{+\infty} d \mathrm{k} k A(\mathrm{k}) A^{\dagger}(\mathrm{k}) \\
& {\left[A^{\dagger}(\mathrm{k}), A\left(\mathrm{k}^{\prime}\right)\right] }=-\delta\left(\mathrm{k}-\mathrm{k}^{\prime}\right) \\
& A^{\dagger}(\mathrm{k}) \mid 0>=0 \\
& E \geq 0
\end{aligned}
$$




\section{Apêndice D}

\section{Álgebra do Tensor Momento-Energia para os Fantasmas}

Neste apêridice, procedemos com os cálculos da álgebra do tensor momento-energia para a contribuição dos fantasmas. Analisamos inícialmente o caso em que o termo cinético possui sinal positivo. O procedimento ó semelhante ao utilizado no apendice $\mathrm{C}$. $O$ tensor momento-energia para os fantasmas neste caso, é dado por (3.99), ou saja

$$
\begin{aligned}
& T_{ \pm \pm}^{(F)}=\frac{1}{2} \partial_{ \pm} c^{ \pm} b_{ \pm \pm}+\frac{1}{4} c^{ \pm} \partial_{ \pm} b_{ \pm \pm} \\
& T_{+-}^{(F)}=0
\end{aligned}
$$

Desejamos provar a seguinte relaşão

$$
\left[: T_{++}(x):,: T_{++}(y)\right]=-i\left(: T_{++}(x):+: T_{++}(y):\right) f^{z}(x-y)+c b^{m}(x-y)
$$

Expandirudo os campos em operadores de aniquilaçăo e criaçăo

$$
\begin{aligned}
& b_{++}(x)=\sqrt{\frac{2}{\pi}} \int_{0}^{+\infty} d \mathbf{k}\left[b(\mathbf{k}) e^{i k x}+b^{\dagger}(\mathbf{k}) e^{-i k x}\right] \\
& c^{+}(x)=\sqrt{\frac{2}{\pi}} \int_{0}^{+\infty} d \mathbf{k}\left[c(\mathbf{k}) e^{i k x}+c^{\dagger}(\mathbf{k}) e^{-i k x}\right]
\end{aligned}
$$


onde

$$
\begin{aligned}
k x & =k^{0} x^{0}+\mathbf{k x} \\
k^{0} & =\mathbf{k}
\end{aligned}
$$

Os momentos canônicamente conjugados aos campos podem ser obtidos a partir da lagrangeana (3.96), ou seja

$$
L=\frac{1}{2 \sqrt{2}} \int d x\left[c^{+} \dot{b}_{++}-c^{+} b_{++}^{\prime}+c^{2} \dot{b}_{\ldots+}+c^{-} b_{--}^{\prime}\right]
$$

obtemos

$$
\begin{aligned}
& \pi_{b_{++}}=-\frac{\partial L}{\partial \dot{b}_{+++}}=\frac{1}{2 \sqrt{2}} c^{+} \\
& \pi_{b_{-+}}=-\frac{\partial L}{\partial \dot{b}_{-+}}=\frac{1}{2 \sqrt{2}} c^{-}
\end{aligned}
$$

Vamos efetuar apenas os cálculos envolvendo a componente $T_{++}^{(F)}$ do tensor, a análise da componente $T_{-}^{(f)}$ se faz de maneira análoga. Usando (D.3) e (D.6) na equação acima obtemos

$$
\pi_{\mathrm{s}_{++}}(x)=\frac{1}{2 \sqrt{\pi}} \int_{0}^{+\infty} d \mathbf{k}\left[c(\mathbf{k}) \mathrm{e}^{i k x}+c^{\dagger}(\mathbf{k}) e^{-i k x}\right]
$$

Devernos ter

$$
\left\{b_{+}+(x), \pi_{b_{++}}(y)\right\}=i v(x-y)
$$

obtemos

$$
\left\{(b), c^{j}\left(\mathbf{k}^{\prime}\right)\right\}=\frac{\frac{k}{2}}{\sqrt{2}} \delta\left(\mathbf{k}-\mathbf{k}^{\prime}\right)
$$

normalizando $c(k)$

$$
c(k) \rightarrow \frac{1}{\sqrt{2}} c(k)
$$


obtemos para os PB's entre os coeficientes das expansöes

$$
\begin{aligned}
\left\{b(\mathbf{k}), c^{\dagger}\left(\mathbf{k}^{\prime}\right)\right\} & =d\left(\mathbf{k}-\mathbf{k}^{\dagger}\right) \\
\left\{b^{\dagger}(\mathbf{k}), c\left(\mathbf{k}^{\prime}\right)\right\} & =i d\left(\mathbf{k}-\mathbf{k}^{\prime}\right) \\
b(\mathbf{k}) \mid 0> & =c(\mathbf{k}) \mid 0>=0
\end{aligned}
$$

Reescrevernos $b_{++}, c^{+}$e $\pi_{b_{++}}$

$$
\begin{aligned}
b_{++}(x) & =\sqrt{\frac{2}{\pi}} \int_{0}^{+\infty} d \mathbf{k}\left[b(\mathbf{k}) e^{i k x}+b^{+}(\mathbf{k}) e^{-i k x}\right] \\
c^{+}(x) & =\frac{1}{\sqrt{\pi}} \int_{0}^{+\infty} d \mathbf{k}\left[c(\mathbf{k}) e^{i k x}+c^{j}(\mathbf{k}) e^{-i k x}\right] \\
\pi_{b_{++}}(x) & =\frac{1}{2 \sqrt{2 \pi}} \int_{0}^{+\infty} d \mathbf{k}\left[c(\mathbf{k}) e^{i k x}+c^{i}(\mathbf{k}) e^{-i k x}\right]
\end{aligned}
$$

As funções $\partial_{+} b_{4+}$ e $\partial_{4} e^{+}$que aparecem no tensor säo dadas por (para $t=0$ )

$$
\begin{aligned}
\partial_{+} c^{+}(x) & =\sqrt{\frac{2}{\pi}} \int_{0}^{+\infty} d \mathbf{k k}\left[c(\mathbf{k}) e^{i \mathbf{k} x}-c^{\dagger}(\mathbf{k}) e^{-i \mathrm{k} x}\right] \\
\partial_{+} b_{++} & =\frac{3 i}{\sqrt{\pi}} \int_{0}^{+\infty} d \mathbf{k k}\left[b(\mathbf{k}) e^{i \mathrm{k} x}-b^{\dagger}(\mathbf{k}) e^{-i \mathrm{k} x}\right]
\end{aligned}
$$

Usando estas equaçōes em $(0.1)$ obtemos para $T_{++}^{(F)}(x)$

$$
\begin{aligned}
: T_{++}^{(F)}(x):= & \frac{i}{3 \pi} \int_{0}^{+\infty} \int_{0}^{+\infty} d \mathbf{k} d \mathbf{p}\left[(2 \mathrm{k}+\mathbf{p}) c(\mathbf{k}) b(\mathbf{p}) e^{i(\mathbf{k}+\mathbf{p}) x}-(2 \mathbf{k}-\mathbf{p}) b^{\dagger}(\mathbf{p}) c(\mathbf{k}) e^{i(\mathrm{k}-\mathbf{p}) z}\right. \\
& \left.-(2 \mathbf{k}-\mathbf{p}) c^{\dagger}(\mathbf{k}) b(\mathbf{p}) e^{-i(\mathbf{k}-\mathbf{p}) x}-(2 \mathbf{k}+\mathbf{p}) c^{\dagger}(\mathbf{k}) b^{\dagger}(\mathbf{p}) e^{-i(\mathbf{k}+\mathbf{p}) x}\right]
\end{aligned}
$$

Para calcular o valor do termo central (constante d em (D.2)) é necessário calcular o elemento de matriz

$$
<0\left|\left[: T_{++}^{F}(x) ;, T_{++}^{(F)}(y)\right]\right| 0>
$$

Substituindo (D.14) am (D.15) obtemos 


$$
\begin{aligned}
& \left.<0\left|\left[: T_{++}^{(F)}(x):: T_{++}^{(F)}(y):\right]\right| 0\right\rangle=\frac{1}{4 \pi^{2}} \int_{0}^{+\infty} \int_{0}^{+\infty} \int_{0}^{+\infty} \int_{0}^{+\infty} d \mathrm{k} d \mathrm{k}^{\prime} d \mathrm{p} d \mathrm{p}^{\prime}
\end{aligned}
$$

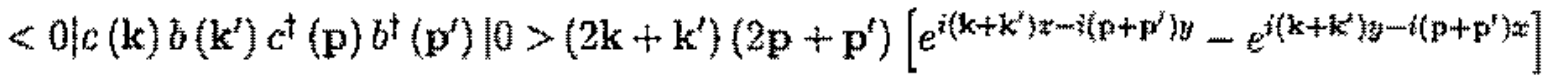

usando (D.11)

$$
\begin{aligned}
& <0\left|\left[: T_{++}^{(n)}(x):, T_{++}^{(5)}(y):\right]\right| 0>=-\frac{1}{4 \pi^{2}} \int_{0}^{+\infty} \int_{0}^{+\infty} \int_{0}^{+\infty} \int_{0}^{+\infty} d \mathrm{k} d \mathbf{k}^{t} d \mathrm{p} d \mathrm{p}^{\prime} \\
& \text {. } \delta\left(\mathbf{k}^{t}-\mathbf{p}\right) \delta\left(\mathbf{k}-\mathbf{p}^{\prime}\right)\left(2 \mathbf{k}+\mathbf{k}^{\prime}\right)\left(2 \mathbf{p}+\mathbf{p}^{\prime}\right)\left[e^{i\left(\mathbf{k}+\mathbf{k}^{\prime}\right) x-i\left(\mathbf{p}+\mathbf{p}^{\prime}\right) y}-e^{i\left(\mathbf{k}+\mathbf{k}^{t}\right) y-i\left(\mathbf{p}+\mathbf{p}^{\prime}\right) x}\right]
\end{aligned}
$$

Integrando sobre $\mathbf{k}^{f}$ e $\mathrm{p}^{\prime}$

$$
\left.<0\left|\left[: T_{++}^{(F)}(x):,: T_{++}^{(P)}(y):\right]\right| 0\right\rangle=-\frac{1}{4 \pi^{2}} \int_{0}^{+\infty} \int_{0}^{+\infty} d \mathrm{k} d \mathrm{p}\left(2 \mathrm{k}+\mathrm{k}^{i}\right)\left(2 \mathrm{p}+\mathrm{p}^{\prime}\right)
$$

Usando a transformaçào

$$
\begin{aligned}
\int_{0}^{+\infty} d \mathbf{k} d \mathbf{k}^{t} f\left(\mathbf{k}, \mathbf{k}^{t}\right) & =\int_{q}^{+\infty} d q \int_{0}^{q} d q^{t} f\left(q-q^{\prime}, q^{\prime}\right) \\
\mathbf{k} & =q-q^{t} \\
\mathbf{k}^{t} & =q^{t}
\end{aligned}
$$

Sscrevemos para (D.17)

$$
\begin{aligned}
<0\left|\left[: T_{+\div}^{(F)}(x):,: T_{++}^{(F)}(y):\right]\right| 0>= & -\frac{1}{4 \pi^{2}} \int_{0}^{+\infty} d q \int_{0}^{q} d q^{z}\left(2 q^{2}+q q^{t}-q^{2}\right) \\
& \cdot\left[e^{i q\{x-y)}-e^{-i g(z-y)}\right]
\end{aligned}
$$

ou 


$$
<0\left|\left[: T_{++}^{(F)}(x):,: T_{++}^{(F)}(y):\right]\right| 0>-\frac{13}{24 \pi^{2}} \int_{0}^{+\infty} d q q^{3}\left(e^{i q(x-y)}-e^{-i q(x-y)}\right)
$$

ou ainda

$$
<0\left|\left[T_{+}(x):, T_{+}^{(F)}(y):\right]\right| 0>=-\frac{13 \dot{i}}{12 \pi} \delta^{n t}(x-y)
$$

Portanto concluimos que

$$
c=-\frac{13 i}{12 \pi}
$$

Vamos agota verificar o primeiro termo do lado direito de (D.2). Utilizamos para este fim o elemento de matriz.

$$
\left.<0\left|\left[: T_{++}^{\langle F\rangle}(x):,: T_{++}^{(F)}(u):\right]\right| q_{1} q_{2}\right\rangle
$$

onde

$$
\left|q_{1} q_{2}>=c^{\dagger}\left(q_{1}\right) b^{\dagger}\left(q_{2}\right)\right| 0>
$$

Usando (D.14) em (D.24)

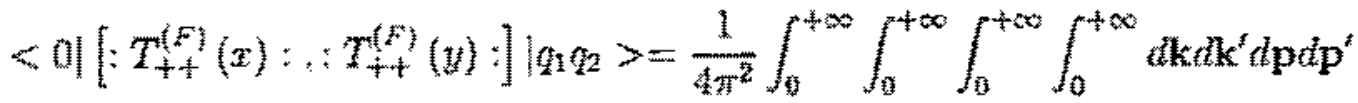

$$
\begin{aligned}
& \text { - }\left(2 \mathbf{k}+\mathbf{k}^{\prime}\right)\left(2 \mathbf{p}-\mathbf{p}^{t}\right) e^{i\left(\mathbf{k}+k^{\prime}\right) x}\left[<0\left|c(\mathbf{k}) b\left(\mathbf{k}^{\prime}\right) b^{\dagger}\left(\mathbf{p}^{\prime}\right) c(\mathbf{p}) c^{\dagger}\left(a_{1}\right) b^{\dagger}\left(q_{2}\right)\right| 0>e^{i\left(\mathbf{p}-\mathbf{p}^{\prime}\right) y}\right. \\
& \left.+<0\left|c(\mathbf{k}) b\left(\mathbf{k}^{\prime}\right) c^{\dagger}(\mathbf{p}) b\left(\mathbf{p}^{\prime}\right) c^{\dagger}\left(q_{1}\right) b^{\dagger}\left\{q_{2}\right)\right| 0>e^{-i\left(\mathbf{p}-\mathbf{p}^{\prime}\right)}\right]-(x \leftrightarrow y) \text {, }
\end{aligned}
$$

onde o termo $(x \leftrightarrow y)$ corresponde a segunda parte do comutador cue corresponde a trocar $x$ por $y$. Usando (D.11) em (D.26) 


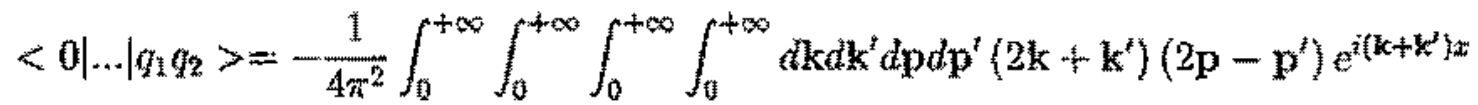

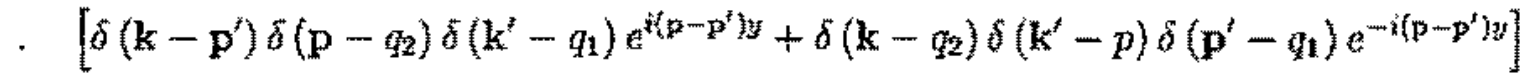

$$
\begin{aligned}
& -(x \rightarrow y)
\end{aligned}
$$

onde $<0|\ldots| q_{1} q_{2}>$ corresponde a (D.24).

Mudando $\mathrm{k}$ por $\mathrm{k}^{t}$ no segundo termo entre colchetes e integrando sobre $\mathrm{p}^{\prime}, \mathrm{p}$ e $\mathrm{k}^{*}$

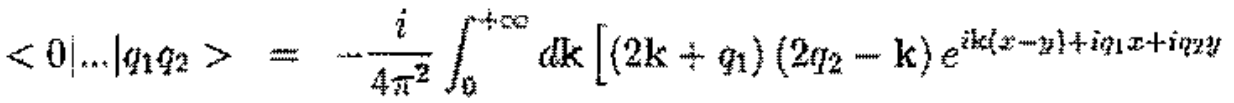

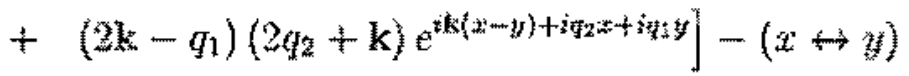

ou desenvolvendo $(x \leftrightarrow y)$

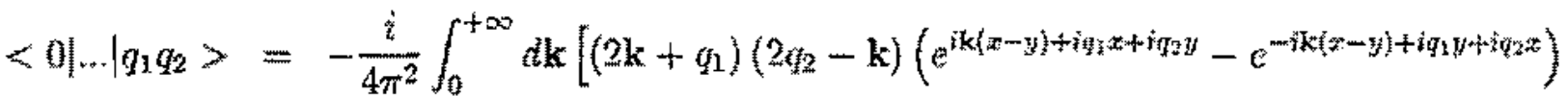

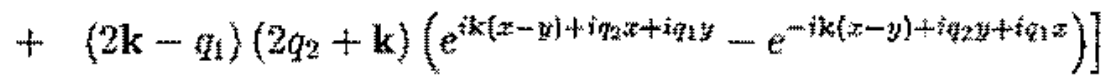

Fatzendo a transformaৎ̧äo $\mathrm{k} \rightarrow-\mathrm{k}^{\prime}$ nos segundos temos das expressöes entre parênteses

$$
\begin{aligned}
& \left\langle 0|\ldots| q_{1} q_{2}\right\rangle=-\frac{i}{\Delta \pi^{2}} \int_{0}^{+\infty} d \mathbf{k}\left\{\left[-2 \mathbf{k}^{2}+\left(4 q_{2}-q_{1}\right) \mathbf{k}+2 q_{1} q_{2}\right] e^{i k(x-y)+q_{1} x+q_{2} y}\right. \\
& +\left[2 k^{2}+\left(4 q_{2}-q_{1}\right) \mathbf{k}-2 q_{1} q_{2}\right] e^{i k\{x-y)+\left(q_{2} x+q q_{1} y\right\}}
\end{aligned}
$$

Utilizando a definiçäo da funçäo delta

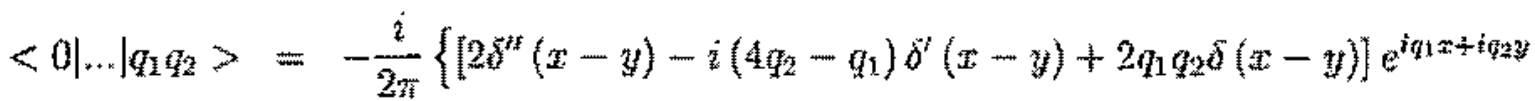

$$
\begin{aligned}
& \left.\div\left[-2 \delta^{n}(x-y)-i\left(4 q_{2}-q_{1}\right) \delta^{t}(x-y)-2 q_{1} q_{2} \delta(x-y)\right] e^{i q_{1} y+\left(q_{2} x\right.}\right]
\end{aligned}
$$


Vamos simplificar esta expressão usando as seguintes relaçó

$$
\begin{aligned}
& \delta^{\prime \prime}(x-y) e^{\mathrm{i} q_{1} x+y_{x} y}=\left(\delta(x-y) e^{q_{2} x}\right)^{\prime \prime} e^{i q_{1} x} \\
& =\delta^{\prime \prime}(x-y) e^{\left.i t_{(q)}+q_{2}\right) x}+2 i q_{2} \hat{\delta}^{z}(x-y) e^{i\left(q_{1}+q_{2}\right) x}-q_{2}^{2} \delta(x-y) e^{i\left(q_{1}+q_{2}\right) x} \\
& \delta^{n}(x-y) e^{i_{1} y+f_{2} x}=\left(\delta(x-y) e^{q_{1} x}\right)^{n} e^{i_{2} x}
\end{aligned}
$$

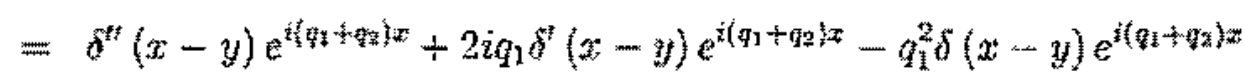

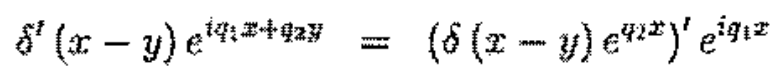

$$
\begin{aligned}
& =\delta^{z}(x-y) e^{i\left(q_{1}+q_{2}\right) x}+i q_{2} \delta(x-y) e^{i\left(q_{1}+q_{2}\right) x} \\
& \delta^{\prime}(x-y) e^{i \eta_{1} y+q_{2} x}=-\partial_{y}\left(\delta\{x-y\} e^{i \eta_{2} y}\right) e^{i \eta_{1} y} \\
& =\delta^{\prime}(x-y) e^{i f\left(q_{1}+\psi_{2}\right) y}-i q_{2} \delta(x-y) e^{i f\left(q_{2}+q_{2}\right) y}
\end{aligned}
$$

Substituindo estas relaçöes em (D.31) obtemos

$$
\begin{aligned}
& <0|\ldots| q_{k} q_{2}>=-\frac{i}{2 \pi}\left\{4 i\left(4 q_{2}-q_{1}\right) \delta^{t}\left(g_{t}-y\right) e^{i\left(q_{1}+g_{2}\right) x}+2\left(q_{1}^{2}-q_{2}^{2}\right) \delta(x-y) e^{i\left(q_{1}+q_{3}\right) x}\right.
\end{aligned}
$$

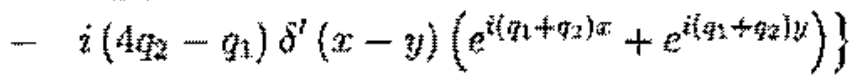

Usando a identidade abaixo no primeiro termo

$$
\begin{aligned}
b^{i}(x-y) e^{i\left(q_{1}+q_{2}\right) z} & =\left(\delta(x-y) e^{i\left(q_{2}+q_{2}\right) x}\right)^{\prime}-\dot{z}\left(q_{1}+q_{2}\right) \delta(x-y) e^{i\left(q_{2}+q_{2}\right) x} \\
& =\delta^{\prime}(x-y) e^{i\left(q_{1}+q_{2}\right) y}-\left(q_{1}+q_{2}\right) \delta(x-y) e^{i\left(q_{2}+q_{2}\right) x}
\end{aligned}
$$

obtemos

$$
<0|\ldots| q_{1} q_{2}>=-\frac{1}{2 \pi}\left(2 q_{2}+q_{1}\right)\left(e^{i\left(q_{1} \div \eta_{2} i x\right.}+e^{i\left(q_{1}+q_{2}\right) y}\right) f^{z}(x-y)
$$


Agora calculamos o elemento de matriz $\left.<0\left|: \eta_{+\leftarrow}^{(F)}(x):\right| q_{1} q_{2}\right\rangle$ para comparar com (D.38) e obtermos a constante $a$. Usando (D.14) obtemos

$<0\left|: T_{4+}^{\left(F^{\prime}\right)}(x):\right| a_{1} q_{2}>=\frac{i}{2 \pi} \int_{0}^{+\infty} \int_{0}^{+\infty} d \mathrm{k} d \mathrm{p}(2 \mathrm{k}+\mathrm{p})<0\left|c(\mathrm{k}) b(\mathrm{p}) c^{\dagger}\left(q_{\mathrm{L}}\right) b^{\dagger}\left(q_{2}\right)\right| 0>e^{i(\mathrm{k}+\mathrm{p}) * x}$

usando as relaçóes de comutaçäo (D.11) podemos escrever

$$
<0\left|: T_{+4}^{(F)}(x):\right| q_{1} g_{2}>=-\frac{i}{2 \pi} \int_{0}^{+\infty} \int_{0}^{+\infty} d \mathbf{k} d \mathbf{p}(2 \mathrm{k}+\mathbf{p}) \delta\left(\mathbf{p}-q_{1}\right) \delta\left(\mathbf{k}-q_{2}\right) e^{i(\mathrm{k}+\mathrm{p}) x}
$$

ou

$$
<0\left|: T_{++}^{(F)}(x):\right| q_{1} q_{2}>=-\frac{i}{2 \pi}\left(2 q_{2}+q_{1}\right) e^{i\left(q_{1}+q_{2}\right) x}
$$

De (D.41) e (D.38) concluimos que a álgebra $(D .2)$ foi satisfeita para o tensor $T(F)(x)$.

Vamos analisar a seguir o efeito da redefinição do vácuo sobre o termo central. Os

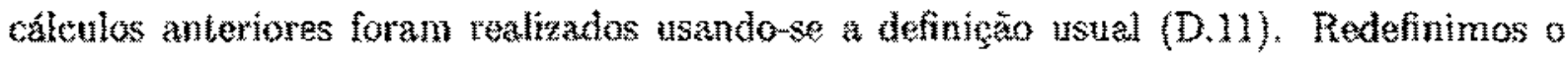
vácuo para

$$
b^{\dagger}(\mathbf{k}) 10>=c^{\dagger}(\mathbf{k}) \mid 0>=0
$$

com esta definiçāo $T_{++}^{\{F}(x)$ é escrito como (veja equaçăo D.14)

$$
\begin{aligned}
: T_{\mathrm{t}}^{\langle F}(x): & =\frac{i}{2 \pi} \int_{0}^{+\infty} d \mathbf{k} d \mathbf{p}\left[(2 \mathbf{k}+\mathbf{p}) c(\mathbf{k}) b(\mathbf{p}) e^{i(\mathbf{k}+\mathbf{p})} x+(2 \mathbf{k}-\mathbf{p}) c(\mathbf{k}) b^{\dagger}(\mathbf{p}) e^{i(\mathbf{k}-\mathbf{p})} x\right. \\
& \left.\left.+(2 \mathbf{k}-\mathbf{p}) b(\mathbf{p}) c^{\dagger}(\mathbf{k}) e^{-i(\mathbf{k}-\mathbf{p}) x}-(2 \mathrm{k}+\mathbf{p}) c^{\dagger}(\mathbf{k}) b^{\dagger}(\mathbf{p}) e^{-i(k+\mathbf{p}) x}\right] \quad \text { (D. } 43\right)
\end{aligned}
$$

O termo central é agora escrito seguindo procedimento similar ao da defini̧̧ăo usual do văcuo como

$$
\begin{aligned}
\left.<0\left|\left[: T_{++}^{(F)}(x):,: T_{++}^{(F)}(y):\right]\right| 0\right\rangle= & -\frac{1}{4 \pi^{2}} \int_{0}^{+\infty} \int_{0}^{+\infty} d \mathbf{k} d \mathbf{p}(2 \mathbf{k}+\mathbf{p})(2 \mathbf{p}+\mathbf{k}) \\
& \cdot\left[e^{-i(\mathbf{k}+\mathbf{p})(x \cdots y)}-e^{i(\mathbf{k}+\mathbf{p})(x-y)}\right]
\end{aligned}
$$


Comparando (D.44) com (D.18) verificamos que existe uma inversão de sinal do termo central. Logo

$$
c=\frac{13 i}{12 \pi}
$$

Vamos analisar agora o efeito da mudança no sinal da energia cinética para o modelo. Invertemos o sinal da açāo (3.96)

$$
L=\frac{1}{2} \int d x c^{\mu} \partial^{\prime \prime} b_{\mu \nu}
$$

Agora o momento canônicamente conjugado a $b_{++}$é dado por

$$
\pi_{b_{++}}=-\frac{1}{2 \sqrt{2}} c^{+}
$$

Portanto para manter

$$
\left\{b_{++}(x), \pi_{b_{++}}(y)\right\}=i \delta(x-y)
$$

devemos ter

$$
\begin{aligned}
\left\{b(\mathbf{k}), c^{\dagger}\left(\mathbf{k}^{\prime}\right)\right\} & =-i \delta\left(\mathbf{k}-\mathbf{k}^{\prime}\right) \\
\left\{b^{\dagger}(\mathbf{k}), c\left(\mathbf{k}^{\prime}\right)\right\} & =-i \delta\left(\mathbf{k}-\mathbf{k}^{\prime}\right) \\
b(\mathbf{k}) \mid 0> & =c(\mathbf{k}) \mid 0>=0
\end{aligned}
$$

O tensor momento-energia (D.1) também muda de sinal mas o termo central mantém o mesmo sinal como pode ser visto de (D.17)

$$
c=-\frac{13 i}{12 \pi}
$$

Se redefinirmos o vácuo para

$$
b^{\dagger}(\mathbf{k})\left|0>=c^{\dagger}(\mathbf{k})\right| 0>=0
$$


verificamos de forma similar á usada no caso anterior que o sinal do termo central é invertido (equaçäo (D.45))

$$
c=\frac{13}{12 \pi}
$$

Para analisarmos os autovalores do operador hamiltoniano usamos as expansōes para $b_{++} e c^{+}$(D.12) na expressão para $H$ (equação 3.104 ). Obtemos

$$
\begin{aligned}
& \left.H=i \int_{0}^{+\infty} d \mathbf{k k}\left\{c^{\dagger}(\mathbf{k}) b(\mathbf{k})+b^{\dagger}(\mathbf{k}) c(\mathbf{k})\right\}\right] \\
& \left\{b(\mathbf{k}), c^{\dagger}\left(\mathbf{k}^{t}\right)\right\}=b \delta\left(\mathbf{k}-\mathbf{k}^{\prime}\right) \\
& \left\{b^{\dagger}(\mathbf{k}), c\left(\mathbf{k}^{x}\right)\right\}=i \delta\left(\mathbf{k}-\mathbf{k}^{\prime}\right) \\
& b(\mathbf{k})|0>=c(\mathbf{k})| \theta>=0
\end{aligned}
$$

aplicando $\mathrm{H}$ sobre um autoestado $\left|p>=b^{\dagger}(p)\right| 0>$, obtemos autovalores E negativos

$$
E \leq 0
$$

Redefinindo o vácuo

$$
\begin{aligned}
& H=-i \int_{0}^{+\infty} d \mathbf{k k}\left[b(\mathbf{k}) c^{\dagger}(\mathbf{k})+c(\mathbf{k}) b^{\dagger}(\mathbf{k})\right] \\
& \left\{b(\mathbf{k}), c^{\dagger}\left(\mathbf{k}^{b}\right)\right\}=i d\left(\mathbf{k}-\mathbf{k}^{t}\right) \\
& \left\{b^{\dagger}(\mathbf{k}), c\left(\mathbf{k}^{t}\right)\right\}=i d\left(\mathbf{k}-\mathbf{k}^{t}\right) \\
& b^{\dagger}(\mathbf{k})\left|0>=c^{\dagger}(\mathbf{k})\right| 0>=0
\end{aligned}
$$

obtemos autovalores pesitivos

$$
E \geq 0
$$

Mudando o sinal da energia cinética e usando a definição usual para o vácuo 


$$
\begin{aligned}
& \left.H=-i \int_{0}^{+\infty} d \mathbf{k} \mathbf{k}\left\{c^{\ddagger}(\mathbf{k}) b(\mathbf{k})+b^{4}\{\mathbf{k}) c(\mathbf{k})\right\}\right]
\end{aligned}
$$

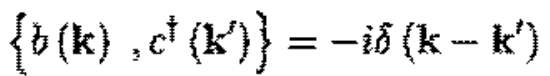

$$
\begin{aligned}
& \left\{b^{t}(\mathbf{k}) \cdot c\left(\mathbf{k}^{t}\right)\right\}=-\hat{b}\left(\mathbf{k}-\mathbf{k}^{t}\right) \\
& \text { (b) } \mid \mathrm{k})>c(\mathrm{k}) \| 0>0 \\
& \varepsilon \leq 0
\end{aligned}
$$

Redefinindo o vắcuo a mantendo a energia cinética com sinal negativo

$$
\begin{aligned}
& H=i \int_{0}^{+\infty} d \mathbf{k} \mathbf{k}\left[b(\mathbf{k}) c^{\dagger}(\mathbf{k})+c(\mathbf{k}) b^{\dagger}(\mathbf{k})\right] \\
& \left\{b(\mathbf{k}), c^{\dagger}\left(\mathbf{k}^{\prime}\right)\right\}=-i \delta\left(\mathbf{k}-\mathbf{k}^{\prime}\right) \\
& \left\{b^{\dagger}(\mathbf{k}), c\left(\mathbf{k}^{\prime}\right)\right\}=-i \delta\left(\mathbf{k}-\mathbf{k}^{\prime}\right) \\
& b^{\dagger}(\mathbf{k})\left|0>=c^{\dagger}(\mathbf{k})\right| 0>=0 \\
& E \geq 0
\end{aligned}
$$

E interessante discutir a noma dos estados no espaço de Holbert, no caso dos fantasmas. Ján qute

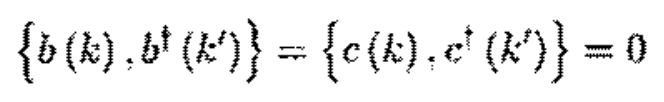

os estados do espaco de Hilbert possuem norma nula 


\section{Apêndice E}

\section{Álgebra do Tensor Momento-Energia para o Campo Escalar + Termo de Improvement}

Neste apêndice analisamos a álgebra quântica do tensor total $T_{++4}^{(T)}$ para o campo escalar com energia cinética positiva mais termo de improvement. O procedinento é identico ao utilizado nos apendices C e D. A lagrangeana neste caso é dada por (de (3.108))

$$
L=-\frac{l}{2} \int d x\left(\partial^{n} \varphi \partial_{\mu} \varphi+Q \partial^{n} \partial_{\mu} \varphi\right)
$$

onde $\mathrm{Q}$ é igual a $Q^{0}$ e $\varphi$ corresponde a $r^{\circ}$ (veja a 3.73 ). Para o fechamento da algebra näo é necessário usar as constantes presentes em (3.73) e (3.108) uma vez que o tensor $T_{\mu}$, pode ser normalizado arbitrariamente.

O tensor momento-energia total nas coordenadas do cone de luz é obtido a partir de (3.94) e $(3.110)$

$$
T_{++}^{(T)}=-\frac{1}{2} \partial_{+} \varphi \partial_{+} \varphi-\frac{1}{2} Q \partial_{+}^{2} \varphi
$$

ou eserevendo de forma abreviada

$$
T_{++}^{(T)}=T_{++}^{(S)}+T_{+}^{(1)}
$$


onde denotamos de $T_{++}^{(s)}$ a contribuição usual do campo escalar e de $T_{++}^{(n)}$ a contribuição do termo de improvement.

Queremos demonstrar a algebra expressa na equaçăo (3.100)

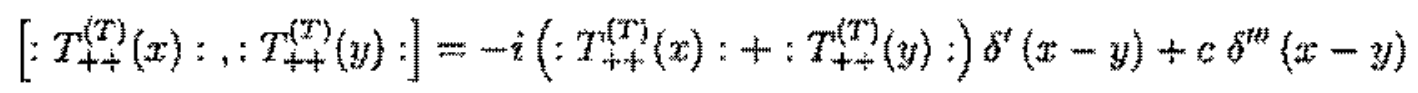

Vamos inicialmente calcular o termo central da álgebra. Para efetuar o cálculo precisamos das expanöes normalizadas dos campos (apêndice $C$ )

$$
\begin{aligned}
\varphi(x) & =\int_{-\infty}^{+\infty} \frac{d \mathrm{k}}{\sqrt{2 \pi}} \frac{1}{\sqrt{2|\mathrm{k}|}}\left[a(\mathrm{k}) e^{i k x}+a^{\dagger}(\mathbf{k}) e^{-i k x}\right] \\
\partial_{+} \varphi(x) & =-i \int_{0}^{+\infty} \frac{d \mathbf{k}}{\sqrt{2 \pi}} \sqrt{\mathrm{k}}\left[A^{\dagger}(\mathbf{k}) e^{-i k x}-A(\mathbf{k}) e^{i k x}\right] \\
\partial_{+}^{2} \varphi(x) & =-\int_{0}^{+\infty} \frac{d \mathbf{k}}{\sqrt{\pi}} k^{\frac{3}{2}}\left[A(\mathbf{k}) e^{i k x}+A^{\dagger}(\mathbf{k}) e^{-i k x}\right]
\end{aligned}
$$

onde foram utilizadas as seguintes convençöes

$$
\begin{aligned}
A(\mathbf{k}) & =a^{\dagger}(-\mathbf{k}) \\
A^{\dagger}(\mathbf{k}) & =a(-\mathbf{k}) \\
{\left[A^{\dagger}(\mathbf{k}), A\left(\mathbf{k}^{\dagger}\right)\right] } & =\left[a(-\mathbf{k}), a^{\dagger}(-\mathbf{k})\right]=\delta\left(\mathbf{k} \cdots \mathbf{k}^{\dagger}\right) \\
A(\mathbf{k}) \mid 0> & =a^{\dagger}(-\mathbf{k}) \mid 0>=0 \\
\mathbf{k} & >0
\end{aligned}
$$

Utilizando as expansōes dadas em (E.5) obtivemos o tensor : $T_{++}^{(5)}(x)$ : para o campo escalar com energia cinética positiva, (conforme mostramos no apêndice $\mathrm{C}$, equação (C.18)

$$
\begin{aligned}
& : T_{++}^{(s)}(x):=\int_{0}^{+\infty} \int_{0}^{+\infty} \frac{d \mathrm{k}^{2} d \mathrm{k}^{t}}{4 \pi} \sqrt{\mathrm{kk}^{t}}\left[A^{\dagger}(\mathrm{k}) A^{\dagger}\left(\mathrm{k}^{\prime}\right) e^{-i\left(\mathrm{k}+\mathrm{k}^{\dagger}\right) x}\right. \\
& \left.-2 A^{\dagger}(\mathrm{k}) A\left(\mathrm{k}^{t}\right) e^{\left.-i t k-k^{j}\right) \mathrm{k}}+A(\mathrm{k}) A\left(\mathrm{k}^{\prime}\right) e^{i\left(\mathrm{k}+\mathrm{k}^{\prime} ; \mathrm{x}\right.}\right]
\end{aligned}
$$


Usando (E.2), (E.5) e (E.6) obtemos: $T_{++}^{(1)}(x)$ :

$$
: T(n):=\frac{Q}{2 \sqrt{\pi}} \int_{0}^{+\infty} d \mathbf{k} \mathbf{k}^{\frac{3}{2}}\left[A(\mathrm{k}) e^{i k z}+A^{\dagger}(\mathrm{k}) e^{-i \mathbf{k} x}\right]
$$

O termo central da álgebra do tensor total é calculado a partix do elemento de matriz

$$
<0\left|\left[: T_{++}^{(T)}(x):,: T_{++}^{(T)}(y):\right]\right| 0>
$$

usando (E.3), escrevemos para o elemento acima

$$
\begin{aligned}
& \quad<0 \mid T_{++}^{(s)}(x):: T_{++}^{(S)}(y):+: T_{++}^{(S)}(x): T_{++}^{(n)}(y): \\
& +: T_{++}^{(I)}(x):: T_{++}^{(S)}(y):+: T_{++}^{(n)}(x):: T_{++}^{(n)}(y): \mid 0>-(x \leftrightarrow y),
\end{aligned}
$$

onde $(x \leftrightarrow y)$ corresponde ao primeiro termo con $x$ trocado por $y$.

O primeiro termo desta expressăo já fó calculado no apêndice $C$ e é dado por (C.25)

$$
<\text { 이 }\left[: T_{++}^{(S)}(x): \leq T_{++}^{(s)}(y): \mid 0>=\frac{1}{48 \pi^{2}} \int_{-\infty}^{+\infty} d q \eta^{3} e^{i g(x-y)}=\frac{i}{24 \pi} \delta^{n t}(x-y)\right.
$$

Usando (E.7) e (E.8) obtemos para o segundo termo

$$
\begin{aligned}
& <0\left|\left[: T_{++}^{(S)}(x)::_{++}^{(n)}(y):\right]\right| 0>=\frac{Q}{8 \pi \sqrt{\pi}} \int_{0}^{+\infty} \int_{0}^{+\infty} \int_{0}^{+\infty} d \mathbf{k} d \mathbf{k}^{\prime} d \mathrm{p} \sqrt{\mathrm{kk}^{\prime}} \mathrm{p}^{\frac{3}{2}} \\
& <0\left|A(\mathrm{k}) A\left(\mathrm{k}^{\prime}\right) A^{\dagger}(\mathrm{p})\right| 0>e^{j\left(\mathrm{k}+\mathrm{k}^{3}\right) \mathrm{x}-\mathrm{ip} y}-(x \rightarrow y)
\end{aligned}
$$

0 elemento de matriz indicado no integrando desta expressăo 6 nulo, logo obtemos

$$
<0\left[: T_{++}^{(g)}(x):,: T_{++}^{(I)}(y):\right] \mid 0>0
$$

Para o terceiro termo de (E.10) obtemos de forma similar 


$$
\begin{aligned}
& <0\left[: T_{++}^{(I)}(x):,: T_{++}^{(S)}(y):\right]\left[0>=\frac{Q}{8 \pi \sqrt{\pi}} \int_{0}^{+\infty} \int_{0}^{+\infty} \int_{0}^{+\infty} d \mathbf{k} d \mathrm{k}^{z} d \mathrm{p} \sqrt{\mathrm{kk}^{\prime}} \mathrm{p}^{\frac{3}{3}}\right.
\end{aligned}
$$

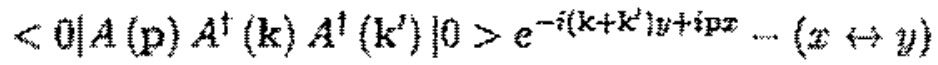

o elemento de matriz indicado no integrando desta expressão também é nulo, logo

$$
<0\left|\left[T_{++}^{(t)}(x):,: T_{++}^{(S)}(y):\right]\right| 0>=0
$$

O quarto termo de (E.10) corresponde a contribuiçāo individual do termo de improverment para o termo central. Usando (E.8)

$$
\begin{aligned}
\left.<00\left[: T_{++}^{(l)}(x):,: T_{++}^{(I)}(y):\right]\right] 0>= & \frac{Q^{2}}{4 \pi} \int_{0}^{+\infty} \int_{0}^{+\infty} d \mathbf{k} d \mathbf{k}^{\prime}\left[\mathrm{k}^{\frac{\gamma}{2}} \mathrm{k}^{\prime \frac{3}{2}}\right. \\
& \left.<0\left|A(\mathrm{k}) A^{\dagger}\left(\mathbf{k}^{\prime}\right)\right| 0>e^{i\left(\mathbf{k} x-\mathbf{k}^{\prime} y\right)}\right]-\{x \leftrightarrow y)
\end{aligned}
$$

Usando as relaçöes de comutał̧ẫo em (E.6)

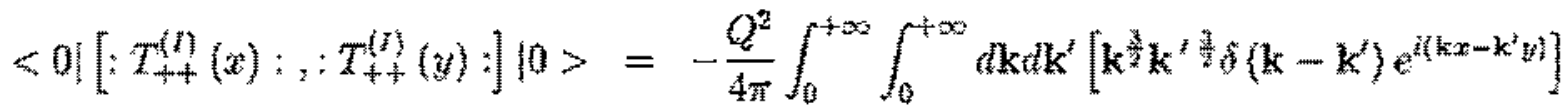

$$
\begin{aligned}
& \text { - }(x \leftrightarrow y)
\end{aligned}
$$

ou integrando em $\mathrm{k}^{z}$ e desenvolvendo $(x \leftrightarrow y)$

$$
<0\left|\left[: T_{++}^{(n)}(x):: T_{++}^{(I)}(y):\right]\right| 0>=-\frac{Q^{2}}{4 \pi} \int_{0}^{+\infty x} d \mathbf{k} d \mathbf{k ~ \mathbf { k } ^ { 3 }}\left[e^{i k(x-y)}-e^{-i \mathbf{k}(x-y)}\right]
$$

ou

$$
<0\left|\left[: T_{++}^{(I)}(x):,: T_{++}^{(I)}(y):\right]\right| 0>=-i \frac{Q^{2}}{2} \delta^{\prime \prime \prime}(x-y)
$$


portanto

$$
c=-\frac{Q^{2}}{2}
$$

Concluimos que para $T_{4+}^{(T)}$ o termo central pode ser obilido da coma de (E.11) e (E.19) sendo dato por

$$
<0 \mid\left[: T_{++}^{(T)}(x):, T_{+}^{(T)}(y): \mid 0>=-\frac{2}{24 \pi}\left(-1+12 \pi Q^{2}\right) b^{m}(x-y)\right.
$$

e neste caso

$$
i=-\frac{i}{24 \pi}\left(-1+12 \pi Q^{2}\right)
$$

Para completar a álgebra do tensor momento-energin precisamos verificar se o puimeiro termo de $\left(\mathrm{E}_{*} 4\right)$ satisfeito. Whilitamos para este fim o elemento de matri\%

$$
<0 \mid[: T+(T)(x):,: T+4):[y>
$$

ot expandindo $\frac{T+1}{4}$

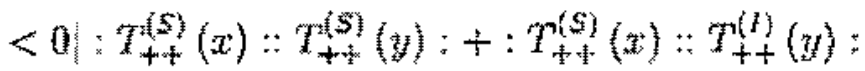

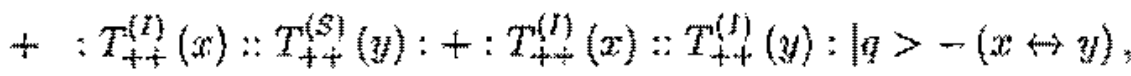

onde

$$
1 q>=A^{\dagger}(q) \mid 0>
$$

Neste caso, usando as expansōes para $T_{+\neq}^{(S)}\left(\right.$ E.T) e $T_{+}^{(t)}$ (E.8) temos o antulamento da primeira da última contribuiçòes, ou seja

$$
\begin{aligned}
& <0\left[\left[T^{(3)}(x):,: T+(y):\right] \mid q>=0\right. \\
& <0\left|\left[: T+(x):: T_{+}^{(n)}(y):\right]\right| q>=0
\end{aligned}
$$


Os outros termos de (E.24) contribuiräo. Para o segundo termo temos

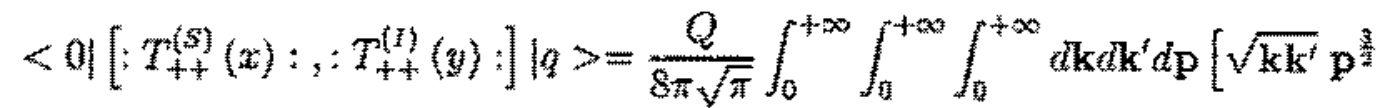

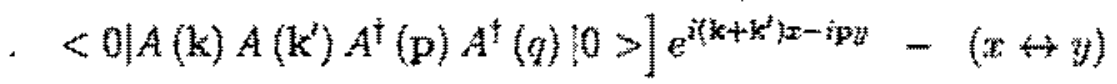

Utilizando as relaçöes de comutagăo (E.B)

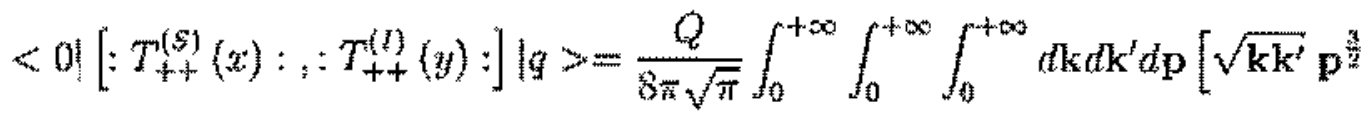

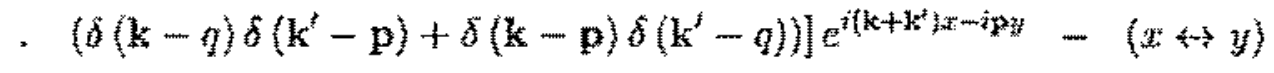

Integrando o primeiro produto de deltas em $\mathbf{p}$ e $\mathbf{k}_{\text {, }}$ o segundo em $\mathbf{p}$ e $\mathbf{k}^{\prime}$ e expandindo $(x \leftrightarrow y)$, obtemos

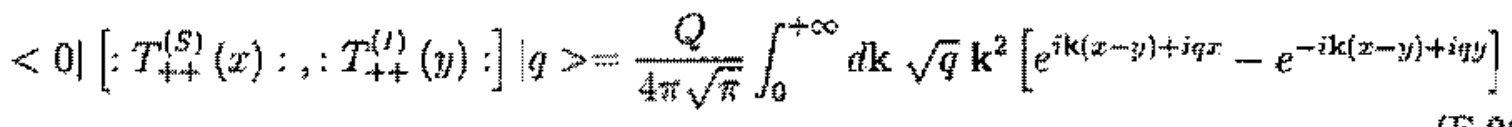

De forma similat obtenos para a lercero temo de (2.24)

$$
<0\left|\left[: T_{++}^{(I)}(x):,: T_{++}^{(S)}(y):\right]\right| q>=\frac{Q}{4 \pi \sqrt{\pi}} \int_{0}^{+\infty} d \mathbf{k} \sqrt{q} \mathbf{k}^{2}\left[e^{-i k(x-y)+i q x}-e^{i \mathbf{k}(x-y)+i \eta y)}\right]
$$

Somandome $(\mathrm{F}, 29)$ e $(\mathrm{E}, 30)$ obtemos o clemento de matriz (E.23)

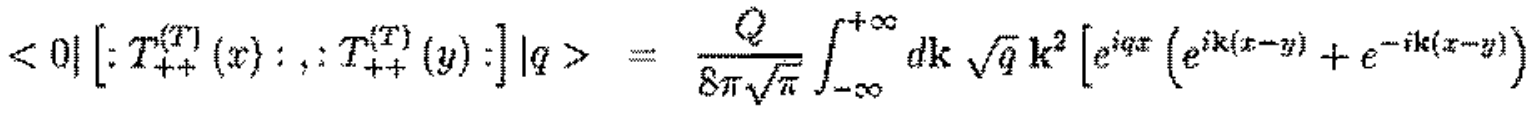

$$
\begin{aligned}
& \left.-e^{i q y}\left(e^{-i k(x-y)}+e^{i k(x-y)}\right)\right]
\end{aligned}
$$

ou, usando a definiçăo da funçẫo delta

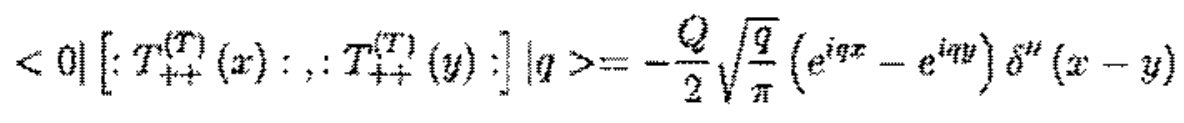


Agora desenvolvemos as relações

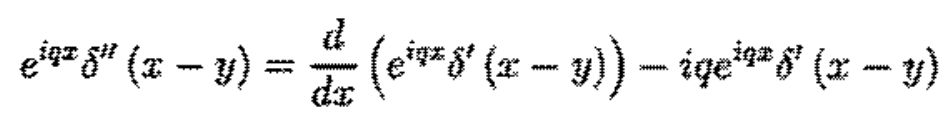

$$
\begin{aligned}
& e^{i n y} \delta^{n}(x-y)=\frac{d^{2}}{d x^{2}}\left(e^{i m y}(x-y)\right)=\frac{d^{2}}{d x^{2}}\left(e^{i x^{2}} \delta(x-y)\right) \\
& =\frac{d}{d x}\left(\hat{q} e^{i g x} \delta(x-y)+e^{i q x} \delta^{z}(x-y)\right) \\
& =\frac{d}{d x}\left(i q e^{i q y} y(x-y)\right)+\frac{d}{d x}\left(e^{i g x} g(x-y)\right) \\
& =\frac{d}{d x}\left(e^{i q x} \delta^{\prime}(x-y)\right)+i q e^{i q y} \delta^{z}(x-y)
\end{aligned}
$$

Usando estas relações em (E.32)

$$
<0\left|\left[: T_{++}^{(T)}(x):,: T_{++}^{(T)}(y):\right]\right| q>=i \frac{Q}{2} \frac{q^{\frac{3}{3}}}{\sqrt{\pi}}\left(e^{i q x}+e^{i q u}\right) \delta^{\prime}(x-y)
$$

Pata verificar a álgebra (E.4) precisamos calcular

$$
<0|: T+(x):| 0\rangle
$$

Una ver que

$$
<0: T+4): 10>=0
$$

precisamos calcular apenas

$$
<0 \mid: T)(x):|a\rangle
$$

De $(\mathrm{E} .8)$

$$
<0\left|: T_{++}^{(I)}(x):\right| q>=\frac{Q}{2 \sqrt{\pi}} \int_{0}^{+\infty} d \mathrm{k} \mathrm{k}^{\frac{3}{2}}<0\left|A(\mathbf{k}) A^{\dagger}(q)\right| 0>e^{i q x}
$$


usando (E.6)

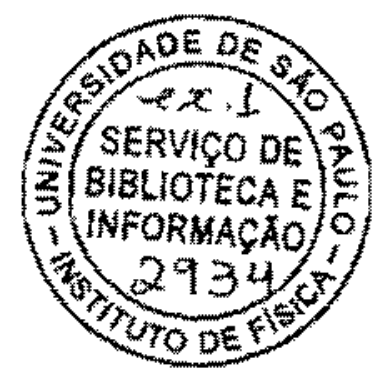

$$
<0\left|: T_{++}^{(1)}(x):\right| x>=\frac{Q}{2 \sqrt{\pi}} q^{2} e^{i q x}
$$

De $(\mathrm{E}, 35)$ e $(\mathrm{E}, 40)$, verificamos que o primeiro temo de (E.4) e satisfeito.

Para completar a álgebra de $T_{++}^{(T)}$, fica faltando obter $T_{+4}^{(5)}$ no segundo membro de (E.4). Este cáloulo é efotuado no apèndice $\mathrm{C}$.

Vamos agora analisar o efeito de uma redefiniçăo do vâuo sobre o termo central para o tensor de improvement. Para o campo escalar este aspecto fo considerado no apêndice C.

Redefinimos o vácuo para

$$
A^{\dagger} \mid 0>=0
$$

com esta definição escrevemos para o termo central de $T_{++}^{(n+}$

$$
\begin{aligned}
& <0\left|\left[: T_{+++}^{(I)}(x):,: T_{++}^{(I)}(y):\right]\right| 0>=\frac{Q^{2}}{4 \pi} \int_{0}^{+\infty} d \mathbf{k} d \mathbf{p ~ k}^{\frac{3}{2}} \mathrm{p}^{\frac{3}{2}}<0\left|A^{\dagger}(\mathbf{k}) A(\mathrm{p})\right| 0>e^{-i(k x-\mathrm{p} y)} \\
& -(x \leftrightarrow y)
\end{aligned}
$$

ou usando as relaçöes de comutaçäo $(\mathrm{E}, 6)$ e expandindo $(x \leftrightarrow y)$

$$
\left.<0\left|\left[: T_{++}^{(n)}(x)::: T_{++}^{(I)}(y):\right]\right| 0\right\rangle=-i \frac{Q^{2}}{2} \delta^{\prime \prime \prime}(x-y)
$$

Portanto com esta definiçăo para o vácuo

$$
c=-i \frac{Q^{2}}{2}
$$

e concluimos que näo houve uma inversão no sinal do temo central om relaf̧̃o ao calculado para definiç̧̃o usual do vécuo. Para analisarmos o efito da redefiniçẫo do vácuo sobre o terno central para o tensor total é necessário considerar a contribuição de $T_{++}^{(S)}$. Chegarnos a conclusăo no apêndice $C$ de que o termo central para o campo escalar tem o sinal invertido com a redefiniçăo para o vắcuo. Assim 


$$
<0\left|\left[: T_{++}^{(T)}(x):,: T_{++}^{(T)}(y):\right]\right| 0>=-\frac{i}{24 \pi}\left(1+12 \pi Q^{2}\right) \delta^{\prime \prime \prime}(x-y)
$$

logo

$$
c=-\frac{i}{24 \pi}\left(1+12 \pi Q^{2}\right)
$$

É importante também analisar o efeito da mudança no sinal da energia cinética do campo escalar, uma vez que possuimos um campo com energia cinética positiva e outro com energia cinética negativa . Conforme vimos no apêndice $\mathrm{C}$ para o campo escalar , uma mudança no sinal da lagrangeana leva a inversão do momento e passa a ser necessário inverter o sinal das relações de comutação. Assim passamos a ter

$$
\begin{aligned}
{\left[A^{\dagger}(\mathbf{k}), A\left(\mathbf{k}^{\prime}\right)\right] } & =\left[a(-\mathbf{k}), a^{\dagger}(-\mathbf{k})\right]=-\delta\left(\mathbf{k}-\mathbf{k}^{\prime}\right\rangle \\
A(\mathbf{k}) \mid 0> & =a^{\dagger}(-\mathbf{k}) \mid 0>=0 \\
\mathbf{k} & >0
\end{aligned}
$$

A lagrangeana para o termo com energia cinética negativa é dada por

$$
L=\frac{1}{2} \int d x\left(\partial^{\mu} \varphi \partial_{\mu} \varphi+Q \partial^{\mu} \partial_{\mu} \varphi\right)
$$

onde $Q$ é igual a $Q^{1}$.

Com a inversão de sinal de $L, T_{++}^{(T)}$ tem o sinal invertido, mas o termo central correspondente muda de valor uma vez que o termo central para o campo escalar $T_{++}^{(S)}$ permanece com o mesmo sinal (apêndice $\mathrm{C}$ ) enquanto para $T_{++}^{(I)}$ o termo central tem o sinal invertido (E.16). Portanto concluimos que invertendo o sinal da lagrangeana obtemos (de (C.50) e (E.20))

$$
c=-\frac{i}{24 \pi}\left(-1-12 \pi Q^{2}\right)
$$

onde usamos a definiçāo usual do vácuo.

Redefinindo o vácuo de acordo com (E.41) obtemos 


$$
c=-\frac{i}{24 \pi}\left(1-12 \pi Q^{2}\right)
$$

Os sinats das normas no espaço de Hilbert para os casos andilsatos neste apendice, sāo os mesmos discutidos no apêndice $C$. 


\section{Bibliografia}

[1] S.W.Hawking, Commun.Math.Phys. 43 (1975) 199.

[2] C.G.Callan, S.B.Giddings, J.A.Harvey e A.Strominger, Phys.Rev. 45D (1902) 1005.

[3] 5.B.Giddings, "Quantum Mechanics of Black Holes", preprint UCSBTH-24w52, 1994.

[4] W.G.Unruh, Phys.Rev. D14 (1976) 870.

[5] P.Hajicek, Commun. Math.Phys. 150 (1992) 545.

[6] Y.Peleg, "Quantum Dust Black Holes", Brandeis U. preprint BRX-TH-350, 1993.

[7] S.B.Giddings, "Black Holes and Quantum Predictability", Proceedings, Quantum Gravity, Nishinomiya, 1902.

[8] J.A.Harvey e A.Strominger, "Quantum Aspects of Black Holes", preprint EFI-92-41, 1992.

[9] Y.Kazama, "On Quantum Black Holes", Lectures given at 13th Symposium on Theoretical Physics: Field Theory and Mathernatical Physics, 1904.

[10] A.Strominger, "Les Houches Lectures on Black Holes", Lectures given at Les Houchea Summer School, Session 62: Fluctuating Geometries in Statistical Mechanics and Field Theory, 1994.

[11] V.Balasubramanian e H.Verlinde, Nucl.Phys, B464, (1996) 213.

[12] J.G.Russo, L.Susskind e L.Thorlacius, Phys.Lett. B292 (1992) 13.

[13] J.Schwinger, Phys.Rev. $82(1951) 664$. 
[14] A.Miković, "Black Holes and Nonperturbative Canonical 2D Dilaton Gravity", preprint IMPERIAL-TP/93-94/16, 1994.

[15] D.Cangemi, R.Jackiw e B.Zwiebach, Ann.Phys. 245 (1996) 408.

[16] L.D.Landau e M.Lifshitz, "The Classical Theory of Fields", Pergamon Press, (1975).

[17] T.Fujiwara, Y.Igarashi o J.Kubo, "Unrecognizable Black Holes in Two Dirnensions", preprint KANAZAWA-93-01, 1993.

[18] K.Sundermeyer, "Constrained Dynamics", Springer Verlag; Heildelberg, (1982).

[19] F.Mandl e G.Shaw, "Quantum Field Theory", John Wiley and Sons, (1986).

[20] P.A.M.Dirac, "Lectures on Quantum Mechanics", Yeshiva University, (1964).

[21] T'Uchino, "Canonical Theory of 2D Gravity Coupled to Conformal Matter", preprint TTT/HEP-216, 1993.

[22] A.Mikovié, Phys Lett. B201 (1992) 19.

[23] A.Miković, Phys.Lett. B304 (1993) 70.

[24] C.Becchi, A.Rouet e R.Stora, Commun.Math.Phys, 42 (1975) 127.

[25] C.Bechi, A.Rouet e R.Stora, Ann.Phys. 98 (1976) 287.

[26] E.S.Fradkin e G.A.Vilkovisky: Phys.Lott. 55B (1975) 224.

[27] I.A.Batalin e G.A.Vilkovisky, Phys.Lete, 60B (10m) 300.

[28] M.Henneaux e C.Teitelboim, "Quantization of Gauge Systems", Princeton University Press; (1992).

[29] M.Henneaux, Phys Rep. 126 No. 1 (1085) 1.

[30] R.Casalbuoni, Nuovo Cimento 33A (1976) 115.

[31] T.Fujiwara, Y.Igarashi e J.Kubo, "Weyl Invariance and Spurious Black Hole in TwoDimensional Dilaton Gravity", Int.J.Mod.Phys. A9 (1994) $4 \$ 11$. 
[32] E.Benedict, R.Jackiw e H.J.Lee, "Functional Schrödinger and BRST Quantization of (1+1)-Dimensional Gravity", Phys.Rev, D54 (1996) 6213.

[33] S. Weinberg, "Gravitation and Cosmology: Principles and Applications of the Theory of Relativity", John Wiley Sons, (1972).

[34] S.Cassemiro F.E. and V.O.Rivelles, "Canonical and Functional Schröedinger Quantization of Two-Dimensional Dilaton Gravity", Submetido para Publicaxão.

[35] K.Kuchar, Phys.Rev.D 39 (1989) 2263.

K.Kuthar and G.Tome, J.Math.Phys. 30 (1089) 1769. 